\title{
Peripheral nervous system control for neuroprostheses
}

\author{
Dissertation \\ for the award of the degree \\ "Doctor rerum naturalium" \\ of the Georg-August-Universität Göttingen \\ within the doctoral program Systems Neuroscience \\ of the Georg-August University School of Science (GAUSS)
}

Submitted by

\section{Jeroen Buil}

from Maastricht, the Netherlands

Göttingen, 2017 



\section{Thesis Committee}

Referee:

Prof. Dr. Hansjörg Scherberger

Neurobiology Laboratory

Deutsches Primatenzentrum GmbH

Kellnerweg 4, 37077, Göttingen

$2^{\text {nd }}$ Referee:

Dr. Igor Kagan

Decision and Awareness Group

Deutsches Primatenzentrum GmbH

Kellnerweg 4, 37077, Göttingen

Prof. Dr. Florentin Wörgötter

Third Institute of Physics - Biophysics

Georg-August-Universität Göttingen

Friedrich-Hund-Platz 1, 37077, Göttingen

\section{Members of the Examination Board}

Prof. Dr. Dr. Hannelore Ehrenreich

Clinical Neuroscience

Max Planck Institute of Experimental Medicine

Hermann-Rein-Straße 3, 37075, Göttingen

Prof. Dr. Ralf Heinrich

Schwann-Schleiden Research Centre, Dept. Cellular Neurobiology

Georg-August-Universität Göttingen

Julia-Lermontowa-Weg 3, 37077, Göttingen

Prof. Dr. Annekathrin Schacht

Affective Neuroscience and Psychophysiology

Georg-August-Universität Göttingen

Goßlerstraße 14, 37073, Göttingen

Date of oral examination: 11th September 2017 
Herewith I declare that I have written this thesis independently and with no other aids and sources than quoted.

Göttingen, 24th August 2017, Jeroen M. M. Buil 


\begin{abstract}
Amputee patients who have lost a hand or arm are severely impaired in their daily life, as they lose the ability to grasp and interact with their environment. While the use of electromyographically controlled prosthetic devices, such as robotic arms, do give back means to grasp objects again, making dexterous movements with them is still difficult and more importantly, they lack the ability to give sensory feedback. The sense of touch is not only critical for making simple movements as tying your shoelaces, but it also plays an important role in emotional communication and the embodiment of the limbs.

Central nervous system interfaces do allow for bidirectional control of prosthetic devices, however they are highly invasive and might give an abstract encoding of the subject's intention. An alternative approach could be to extract movement information from the peripheral nervous system (PNS) instead. Beside the reduction of invasiveness, it could also greatly improve decoding, as PNS electrodes will record the direct feed to the muscles, and thereby could avoid the perhaps more complex signals of the CNS. Aside from that, stimulating the PNS, instead of the CNS could evoke more naturally perceived sensations of lost limbs. Even in forearm amputations the neural pathways are still preserved, potentially making PNS interfaces excellent candidates for bidirectional control of motor prosthetics. Recent development in electrode fabrication allows the production of very fine multichannel wire electrodes that can be inserted into the nerves.
\end{abstract}

In this thesis, I investigated if a bidirectional prosthetic interface can be achieved using PNS arrays implanted in the medial and ulnar nerve in the upper limb of a rhesus macaque (Macaca mulatta). This was done done with Transverse Intrafascicular Multichannel Electrodes (TIMEs), which are 12 channel, thin-film electrode arrays capable of recording and stimulating individual nerve fascicles. Two acute and one short-term experiment with non-human primates (NHP) showed that it is feasible to implant TIMEs in the PNS of a rhesus macaque. With the surgical procedure established, two long-term implantations were performed with two TIMEs in a fully trained animal.

The long term implantations were a success with respect to the fact that the animal recovered quickly with a total absence of paralysis and/or lack of function. Unfortunately the electrode 
lifetime was rather limited. In the first implantation the median and ulnar TIMEs lasted 2 and 5 months, respectively. In the second implantation they lasted only 2 and 3 weeks.

During the long term implantations the TIME's ability to record neural activity from the median and ulnar nerve was tested, as well as the ability to stimulate the nerve to evoke sensory percepts. This was done in two distinct behavioural tasks. The first was a motor decoding task, in which the animal grasped and lifted a wide variety of objects on a turn table, while simultaneously the neural signals were recorded and the hand kinematics were tracked. After the recording period the animal performed a somatosensory discrimination task with either tactile cues applied to the hand or electrical stimulation to the nerves.

The signal-to-noise ratio of the neural recordings was poor and in only a few recordings we were able to detect spiking activity. However it was too sparse for successful decoding of the performed grip type. The long electrode cable under the skin in combination with a dynamic task design introduced too many movement artefacts in the signal. The short lifetime of the electrodes also affected the ability to train the somatosensory discrimination task with electrical stimulation to the nerves. The animal was successfully trained in both the motor decoding task and the somatosensory discrimination task with tactile stimulation.

To continue this line of research it would be necessary to move towards a solution with at least an implantable amplifier close to the recording site and preferably also be completely wireless. This would greatly improve the signal-to-noise ratio in the neural recordings and thus the ability to detect and decode neural activity. The TIME in its current form is not stable enough for long term implantation and thus for investigating somatosensory stimulation. Last but not least, while the macaque model is sufficient for basic research and the establishment of stimulation methods, the more detailed exploration of somatosensory restoration (such as different sensory percepts) will necessarily require to move to human subjects (or patients) in order to obtain oral feedback about the elicited percepts. 



\section{Acknowledgements}

First, I want to thank Hans Scherberger for the opportunity, support, and trust he has placed in me throughout these years. As mathematician, surgeon, and neurobiologist, he inspired this fellow "Jack off all trades" that limiting yourself to a single craft is not necessary for success. Furthermore, I am grateful to my thesis committee members, consisting of Hans, Igor Kagan and Florentin Wörgötter, for the helpful scientific criticism and the friendly atmosphere during our meetings.

Support also came from all my former and current lab members, with special thanks to our TA's Natalie, Sabine and Matthias, for their unrelenting monkey/setup support and for filling the hallways with laughter. All you guys made the lab a good place to be.

Good distraction outside of work is the key to a good life balance and my friends in close and far away places were happy to provide this. Thank you Movie Nighters, the Roko-group and the Heinrich-gang, you know who you are and believe me that I do not take your friendship for granted. A special thanks goes to my "gymbro's" Jonathan and Ben, because they did not only train me physically, but strengthened me mentally as well (not to mention the important life lessons they taught me about soap). My thanks also goes to Arjan for all our 'Adventure Time': a well needed life-boost to get my spirits back up.

Thank you Astrid, for the gentle words in time of need.

Thanks to music, how silly it may sound, but those who know me, know that music is an always audible factor in my life and allows me to express myself when words can't.

Thank you to my family for supporting my decisions.

Last but not least, thank you Zara, I do not take the sacrifice you had to make lightly.

What I am trying to say is that the road to a $\mathrm{PhD}$ is not something one walks alone.

Some people came into my life, while others left. Some left behind big impressions, while others short experiences. Some are named here, while others are not. But all got me to this point in life where I now stand and shaped me into the person I am.

Thank you. 
'And at once I knew, I was not magnificent High above the highway aisle

Jagged vacance, thick with ice

But I could see for miles, miles, miles...'

\section{- Bon Iver}





\section{Contents}

\begin{tabular}{lll}
\hline Abstract & iii
\end{tabular}

\begin{tabular}{|l|l}
\hline Abbreviations & xv
\end{tabular}

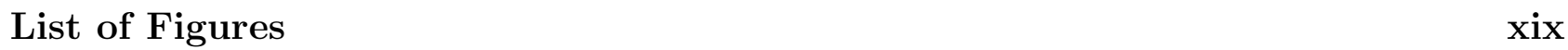

\begin{tabular}{ll}
\hline List of Tables & xxiii
\end{tabular}

\begin{tabular}{llr}
\hline & Introduction & 1
\end{tabular}

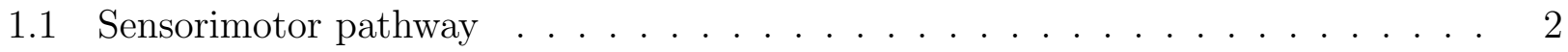

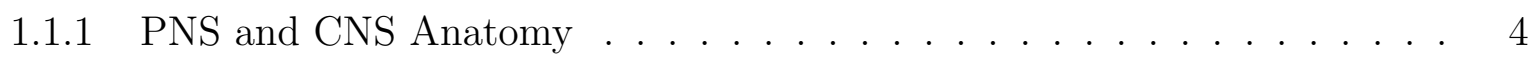

1.2 Rehabilitation strategies $\ldots \ldots \ldots \ldots \ldots \ldots$

1.2 .1 Neural interfaces for motor restoration . . . . . . . . . . . . . . . . 10

1.2 .2 Sensory restoration $\ldots \ldots \ldots \ldots \ldots \ldots \ldots$

$1.2 .3 \quad$ Sensory substitution $\ldots \ldots \ldots \ldots \ldots \ldots$

1.2 .4 Targeted sensory reinnervation $\ldots \ldots \ldots \ldots$

$1.2 .5 \quad$ Nerve interfaces $\ldots \ldots \ldots \ldots \ldots \ldots$

1.3 Motivation and objectives of this thesis . . . . . . . . . . . . . . . 20 
2 Materials and Methods 23

2.1 Neural interface . . . . . . . . . . . . . . . . . . . . . . . . . 24

$2.1 .1 \quad$ Transverse Intrafascicular Multichannel Electrodes (TIME) . . . . . . . . 24

2.1 .2 Implantation techniques $\ldots \ldots \ldots \ldots \ldots$

$2.1 .3 \quad$ Surgical procedure - Long-term experiments . . . . . . . . . . . . . . 27

2.1 .4 Deplantation of TIME arrays $\ldots \ldots \ldots \ldots \ldots$. . . . . . . . . 27

2.2 Monitoring electrode longevity $\ldots \ldots \ldots \ldots$

2.3 Motor Decoding task $\ldots \ldots \ldots \ldots$

$2.3 .1 \quad$ Basic procedures $\ldots \ldots \ldots \ldots \ldots \ldots$

$2.3 .2 \quad$ Experimental setup . . . . . . . . . . . . . . . . . . . . . . . 29

2.3 .3 Behavioural paradigm . . . . . . . . . . . . . . . . . . 34

2.3 .4 Neural and EMG recordings $\ldots \ldots \ldots \ldots$. . . . . . . . . . . . . . . 35

$2.3 .5 \quad$ Neural signal analysis . . . . . . . . . . . . . . . . . . . . . 35

2.3 .6 Object Classification $\ldots \ldots \ldots \ldots \ldots \ldots$

2.4 Somatosensory Discrimination Task $\ldots \ldots \ldots$. . . . . . . . . . . . . . . . . . . 38

$2.4 .1 \quad$ Basic procedures $\ldots \ldots \ldots \ldots \ldots$

$2.4 .2 \quad$ Experimental setup . . . . . . . . . . . . . . . . . . . . 38

2.4 .3 Behavioural paradigm . . . . . . . . . . . . . . . . . . . 44

$2.4 .4 \quad$ Somatosensory channel mapping . . . . . . . . . . . . . . . . . . . . 48

2.4 .5 Data analysis $\ldots \ldots \ldots \ldots \ldots$

2.4 .6 Electrical stimulation . . . . . . . . . . . . . . . . . . . . 50 


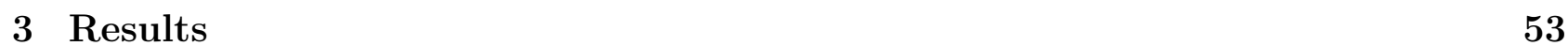

3.1 Implantation techniques $\ldots \ldots \ldots \ldots \ldots \ldots \ldots$

3.1.1 Acute electrical stimulation with cuff and TIME electrodes . . . . . . . . 53

$3.1 .2 \quad$ Acute stimulation and recording with TIME electrodes . . . . . . . . . . 54

$3.1 .3 \quad$ Short-term implantation . . . . . . . . . . . . . . . . 56

3.1 .4 Intraneural recordings - First tests $\ldots \ldots \ldots \ldots$. . . . . . . 57

$3.1 .5 \quad$ Electromagnetic field influence on recording quality . . . . . . . . . . . . 58

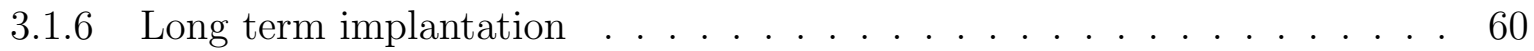

3.1 .7 Deplantation of TIME arrays $\ldots \ldots \ldots$. . . . . . . . . . . 61

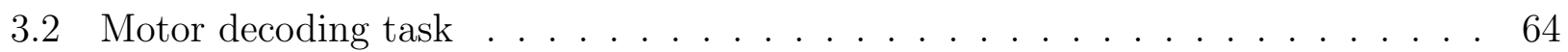

3.2.1 High noise levels and movement artefacts . . . . . . . . . . . . . . 64

3.2 .2 Neural activity . . . . . . . . . . . . . . . . 67

3.3 Somatosensory discrimination task . . . . . . . . . . . . . . . . 73

$3.3 .1 \quad$ Vibration only results $\ldots \ldots \ldots \ldots \ldots$. . . . . . . . . . . . . . . .

$3.3 .2 \quad$ Electrical stimulation only $\ldots \ldots \ldots \ldots \ldots$. . . . . . . . . . . 74

3.4 Electrode degradation $\ldots \ldots \ldots \ldots \ldots$. . . . . . . . . . . . . . . . . . .

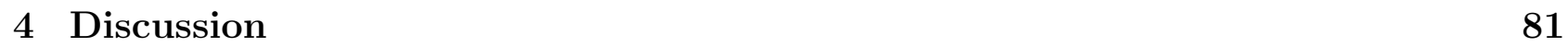

4.1 Implantation techniques $\ldots \ldots \ldots \ldots$. . . . . . . . . . . . . . . . . . 81

4.1 .1 General aspects . . . . . . . . . . . . . . . . . . 81

4.1 .2 Non-human primate aspects . . . . . . . . . . . . . . . 83

4.2 Recording quality . . . . . . . . . . . . . . . . . . . . . . 84

4.3 Somatosensation task . . . . . . . . . . . . . . . . . . . 87 
4.4 Electrode longevity $\ldots \ldots \ldots \ldots$. . . . . . . . . . . . . . . . . 90

4.5 Outlook $\ldots \ldots \ldots \ldots \ldots$

$\begin{array}{llr}5 & \text { Conclusion } & 95\end{array}$

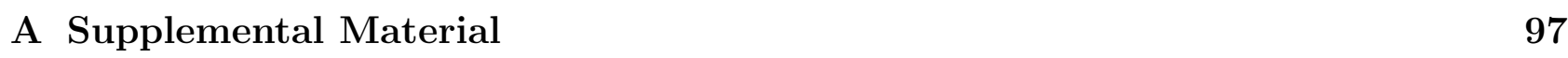

A.1 Vibration motor analysis . . . . . . . . . . . . . . . . . . . . 97

A.1.1 Sound analysis . . . . . . . . . . . . . . . . . . . . . . . . 97

A.1.2 Force sensing resistors . . . . . . . . . . . . . . . . . 98

A.1.3 Motor Results . . . . . . . . . . . . . . . . . . . . . . . . 101

A.2 Somatosensory discrimination task . . . . . . . . . . . . . . . 103

A.2.1 Performance Chart - Vibration Only . . . . . . . . . . . . . . 103

\begin{tabular}{ll}
\hline B Side Project - Releasable Collar & 105
\end{tabular}

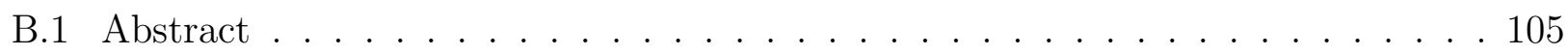

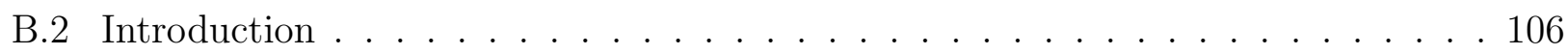

B.3 Methods . . . . . . . . . . . . . . . . . . . . . . 106

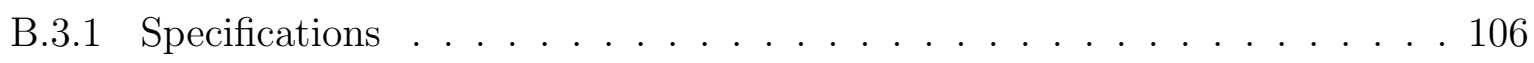

B.4 Hardware . . . . . . . . . . . . . . . . . . . . . . . . . . . 108

B.4.1 Locking Mechanism . . . . . . . . . . . . . . . . . . . . 108

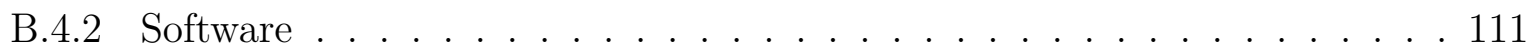

B.4.3 Power consumption . . . . . . . . . . . . . . . . . . . . . . . 111

B.5 Discussion \& Conclusion $\ldots \ldots \ldots$. . . . . . . . . . . . . . . . . . 113

\begin{tabular}{lr}
\hline Bibliography & 117
\end{tabular} 
C Curriculum Vitae 



\section{Abbreviations}

$\mathrm{API}=$ Application Programming Interface

$\mathrm{BMI}=$ Brain Machine Interface

$\mathrm{CBI}=$ Computer Brain Interface

$\mathrm{CMA}=$ Cingulate Motor Area

$\mathrm{CNS}=$ Central Nervous System

DOF $=$ Degrees Of Freedom

$\mathrm{ECG}=$ Electrocardiography

$\mathrm{EMG}=$ Electromyography

$\mathrm{ENG}=$ Electroneurography

FA1 $=$ Fast Adapting Fibres Type I

FA2 $=$ Fast Adapting Fibres Type II

GUI $=$ Graphical User Interface

$\mathrm{HPF}=$ High-Pass Filter

$\mathrm{I} / \mathrm{O}=$ Input/Output

ICMS $=$ Intracortical Micro Stimulation

LDA $=$ Linear Discriminant Analysis

$\mathrm{LPF}=$ Low-Pass Filter

$\mathrm{M} 1=$ Motor Cortex

PCA $=$ Principal Components Analysis

PNS $=$ Peripheral Nervous System

$\mathrm{PM}=$ Premotor Cortex

$\mathrm{S} 1=$ Sensory Cortex

SA1 $=$ Slow-Adapting Fibres Type I

SA2 $=$ Slow-Adapting Fibres Type II

SMA = Supplementary Motor Area

TIME $=$ Transverse Intrafascicular Multichannel Electrode

$\mathrm{VI}=$ Virtual Instrument

$\mathrm{UDP}=$ User Datagram Protocol 



\section{List of Figures}

1.1 NervousSystem . . . . . . . . . . . . . . . . . . . . . 5

2.1 Schematic design of the TIME electrode. . . . . . . . . . . . . . . . . . . . 24

2.2 Overview of complete TIME array $\ldots \ldots \ldots \ldots \ldots \ldots$

2.3 Motor-Decoding Task . . . . . . . . . . . . . . . . . . . . . . . 31

2.4 LabVIEW VI-Decoding Task . . . . . . . . . . . . . . . . . . . . . . . . 31

2.5 Setup Overview - Motor $\ldots \ldots \ldots$. . . . . . . . . . . . . . . 32

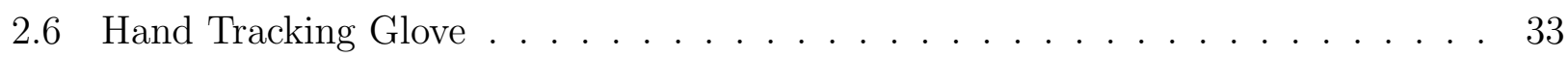

2.7 Data Pre-Processing $\ldots \ldots \ldots \ldots \ldots \ldots$

2.8 SpikeSorting . . . . . . . . . . . . . . . . . . . . . . . 37

2.9 Vibration Glove $\ldots \ldots \ldots \ldots$

2.10 Vibration Motor $\ldots . \ldots \ldots$. . . . . . . . . . . . . . . . 41

2.11 Wiring Schematic Vibration Motor . . . . . . . . . . . . . . . . . . . 42

2.12 LabVIEW VI-Sensory Task . . . . . . . . . . . . . . . . . . 43

2.13 Setup Overview - Sensory $\ldots \ldots \ldots$. . . . . . . . . . . . . . . 44

2.14 CereStim GUI . . . . . . . . . . . . . . . . . . . . . . . . . . . . 45

2.15 Graspbox Sensory Task . . . . . . . . . . . . . . . . . . . . . 46

2.16 Somatosensory Discrimination Task . . . . . . . . . . . . . . . . . . . . 48 
3.1 Cuff electrode implantation . . . . . . . . . . . . . . . . . . . 55

3.2 TIME array implantation $\ldots \ldots \ldots \ldots \ldots \ldots$

3.3 Recording of cuff electrode stimulation . . . . . . . . . . . . . . . . 55

3.4 Recording Sample . . . . . . . . . . . . . . . . . . . . 57

3.5 Noise level scatter plot. . . . . . . . . . . . . . . . . . . . 58

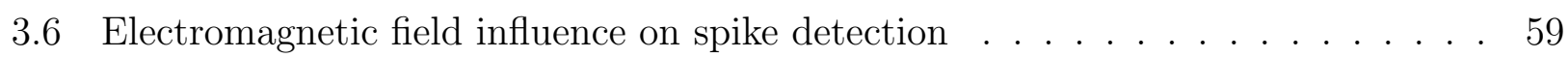

3.7 Deplantation of TIME arrays $\ldots \ldots \ldots \ldots$. . . . . . . . . . . . . 62

3.8 Histology of TIME arrays $\ldots \ldots \ldots$. . . . . . . . . . . . . . . . 63

3.9 Filtering Steps.$\ldots \ldots \ldots \ldots$

3.10 Filtering Steps $\ldots \ldots \ldots \ldots$. . . . . . . . . . . . . . . . 66

3.11 PSTH Motor Task $\ldots \ldots \ldots$. . . . . . . . . . . . . . . . . . . . . . 68

3.12 Confusion Matrices $\ldots \ldots \ldots \ldots$. . . . . . . . . . . . . . . 68

3.13 Filtering Steps . . . . . . . . . . . . . . . . . . . 70

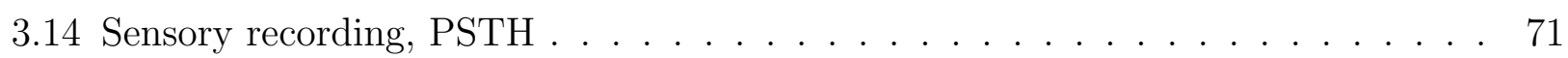

3.15 Sensory recording, Example waveform . . . . . . . . . . . . . . . . . 71

3.16 Powerband $\mathrm{PSTH}$. . . . . . . . . . . . . . . . . . . . . 72

3.17 Reaction Time . . . . . . . . . . . . . . . . . 74

3.18 Psychometric Curve - Vibr Only . . . . . . . . . . . . . . . . . . . 75

3.19 Impedances of 1 st Implantation $\ldots \ldots \ldots$. . . . . . . . . . . . . 78

3.20 Impedances of 2 nd Implantation . . . . . . . . . . . . . . . . . . . 78

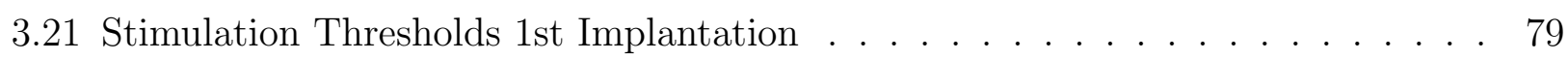

3.22 Stimulation Thresholds 2 nd Implantation . . . . . . . . . . . . . . . . . . . . 79 
A.1 Force Sensing Resistor (FSR) $400 \ldots \ldots$. . . . . . . . . . . . . . . . . . 99

A.2 Motor holding apparatus 'Contingentia v1.1' . . . . . . . . . . . . . . 100

A.3 Wiring schematic of vibration motor analysis setup . . . . . . . . . . . 101

A.4 Motor frequency consistency . . . . . . . . . . . . . . . . . . . . . . 102

A.5 Performance Chart Somatosensory discrimination task - Vibration Only . . . . . 103

B.1 Releasable Collar . . . . . . . . . . . . . . . . . . . . . . . . 107

B.2 Wiring Schematic - Releasable Collar . . . . . . . . . . . . . . . . . . . . . . 109

B.3 Locking Mechanism . . . . . . . . . . . . . . . . . . . . . . . . . . 110

B.4 Schematic Drawing - Lock Mechanism . . . . . . . . . . . . . . . . . . . 110

B.5 Android GUI - Collar . . . . . . . . . . . . . . . . . . . . . . . . . 112 



\section{List of Tables}

2.1 Turn Table Objects . . . . . . . . . . . . . . . . . . . . 30

2.2 Tracked Degrees of Freedom - Hand tracking system . . . . . . . . . . . . . . . . 34

2.3 Technical specifications - Vibration motor (Swissphone Quattro Hurricane) . . . 41

A.1 Specifications - Force Sensing Resistor (FSR) 400 . . . . . . . . . . . . . . . . . 99

B.1 Releasable Collar - Part List . . . . . . . . . . . . . . . . . . . . . . . 109

B.2 Power consumption chart - Arduino . . . . . . . . . . . . . . . . . 113 



\section{Chapter 1}

\section{Introduction}

With this introduction I hope to give a glimpse of the current state in the fields of motor neurorehabilitation and sensory neurorehabilitation, including the current challenges in these fields. To tackle two such seemingly diverse topics I start by giving an overview of the sensorimotor pathway to show that these two fields are more intertwined than one might initially think. Once we know how the system should function, we can then explore what happens when it does not like for example, in case of an amputation or paralysis. The type of lesion of the system will determine what type of interface can be used to (partly) restore its function. I will highlight interface examples with different levels of invasivity, ranging from non-invase (electromyography) to very invasive interfaces (brain electrode arrays). Particular focus will be on the invasive types of peripheral nervous system interfaces. Finally I want to outline the limitations of the state of the art motor prosthetics interfaces as well as the sensory restoration and substitution techniques to show that peripheral nerve interfaces could contribute to the improvement of these techniques.

Considering both my personal interest and the focus of this $\mathrm{PhD}$ project, this introduction will be less focused on the biological/anatomical side, but instead more on the technical aspects and challenges: How can we interact with the nervous system and what are the technical challenges involved with it? 


\subsection{Sensorimotor pathway}

The ability to control our hands with such high dexterity as humans do is unique in the animal kingdom. It is a combination of the ability to coordinate the multitude of skeletal muscles connected to the bones making up our limbs, and the ability to feel changes in our environment. Dexterous hand control is essential in our daily life, which becomes especially apparent when one loses a hand or arm. Not only does one lose the means to move and interact with the environment, the sense of touch also plays a crucial role in the embodiment of our body and, no less importantly, in emotional communication.

Neuroprosthetic devices are therefore a highly desired technology as they currently give amputee patients the ability to grasp and manipulate objects again. However, especially hand prostheses have much room left for improvement. Controlling several degrees of freedom (DOF) at the same time is still challenging, which makes dexterous movements cumbersome or even infeasible, especially considering the fact that an intact hand-arm system has about 27 DOF. Another important matter is that sensory feedback is often completely neglected when attempting to restore a limb. The focus lies mostly on restoring more and more DOF. However, the motor system relies heavily on sensory feedback and taking this into account could not only drastically improve the control of prosthetic devices, but also the acceptance and embodiment of the artificial limb for the patient. Especially the latter could use improvement, as we see that many prosthetic users stop using their device after a while Kejlaa, 1993, Dhillon and Horch, 2005, Carrozza et al., 2006.

Even the simplest reach-and-grasp movement hides a very complicated feedback system. To emphasise the complexity of human grasping and how heavily it depends on sensory feedback, I want to walk through a simple example: Imagine grasping a screwdriver out of your toolbox, which is filled with a variety of other tools. On first glance it might seem like it just requires the brain to send a command to the muscles in the arm to move the hand around in space and subsequently close the hand around the screwdriver. However, even before a single movement is made, it is preceded by an elaborate planning process and this plan is continuously monitored and corrected during execution. To name a few steps: in order for the brain to make a movement 
plan, it gathers information from both the visual system and memory about the object you wish to grasp (shape, weight, size, orientation and location in space), makes a movement plan to reach the object from your hand's current position, and preshapes the hand accordingly in order to grasp it correctly. Once the movement is initiated, the brain is not only in charge of operating the muscles in the arm and hand. As you are moving your arm in space, your center of gravity will shift, which requires, amongst others, muscles in your back to flex in order to prevent you from tipping over. This process is continuously corrected on the way to the goal as there might be external forces acting on the body. When the hand has reached its desired position and you start 'feeling around' in the bag in search for the screwdriver, the mechanoreceptors in the skin of the hand give feedback about the shape, texture and weight of whatever it is touching, which allows the brain to identify if it is the desired object. Finally, when the object is found and can be grasped, it is crucial to grasp it with the correct amount of force so it doesn't slip out of the hand while retrieving it.

I will use this example to give a basic run-through of the sensorimotor pathway, which best can be seen as a loop. Starting in the brain a motor plan is devised and commands are sent through the nerves to the muscles that move the hand and body. Sensory receptors detect the changes in the body and the environment, caused by said motor commands and send feedback through the nerves back to the brain where the plan can be revised and the loop starts again. With this example I hope to show that seemingly simple actions require a complex system that does not only involve motor planning and executing, but is also strongly dependent on information it receives from the wide variety of sensors in our body. 


\subsubsection{PNS and CNS Anatomy}

As Marieb and Hoehn, 2007] stated:

'The human brain, for all its sophistication

would be useless without its links to the outside world.'

This, in my opinion, shows that you cannot just view the brain as an isolated structure. When we talk about the central nervous system (CNS) we refer to the brain, cerebellum and spinal cord, while the peripheral nervous system (PNS) includes all other neural structures. A diagram is shown in Figure 1.1. The focus in neuroscience often lies on what happens in the CNS, but one tends to forget that without the input to and output from the PNS, the central nervous system would not function. On the other hand a system of standalone bundle of (PNS) nerves, as found with simple organisms like jellyfish, would not allow complex tasks either. It is the combination and interaction of the CNS and PNS that allows us vertebrates to function the way we do.

If we go back to our idea of grasping a screwdriver. Before the motor cortex (M1) sends the motor signals down to the muscles, a movement plan is formed. For this, M1 receives input from a wide range of other cortical areas, like the premotor cortex (PM), parietal cortex (area 5), somatosensory cortex (S1), supplementary motor area (SMA), cingulate motor area (CMA), and subcortical brain areas like the basal ganglia, cerebellum and the thalamus Borra et al., 2008. More on these latter three areas will be said later as they involve information from somatosensory receptors. Depending on the type of movement that is executed, different cortical areas are involved in the preparation of the motor plan: e.g. while pre-trained or mentally rehearsed movements involve the SMA, grabbing the screwdriver in our example involves the premotor cortex, which receives input from the visual cortex about the location, shape, size, orientation and weight of the object [Murata et al., 1997, Borra et al., 2010]. When grasping an object it may not seem self-evident how much an object probably weighs, but from experience you will know how much a screwdriver will weigh and therefore you will not use 


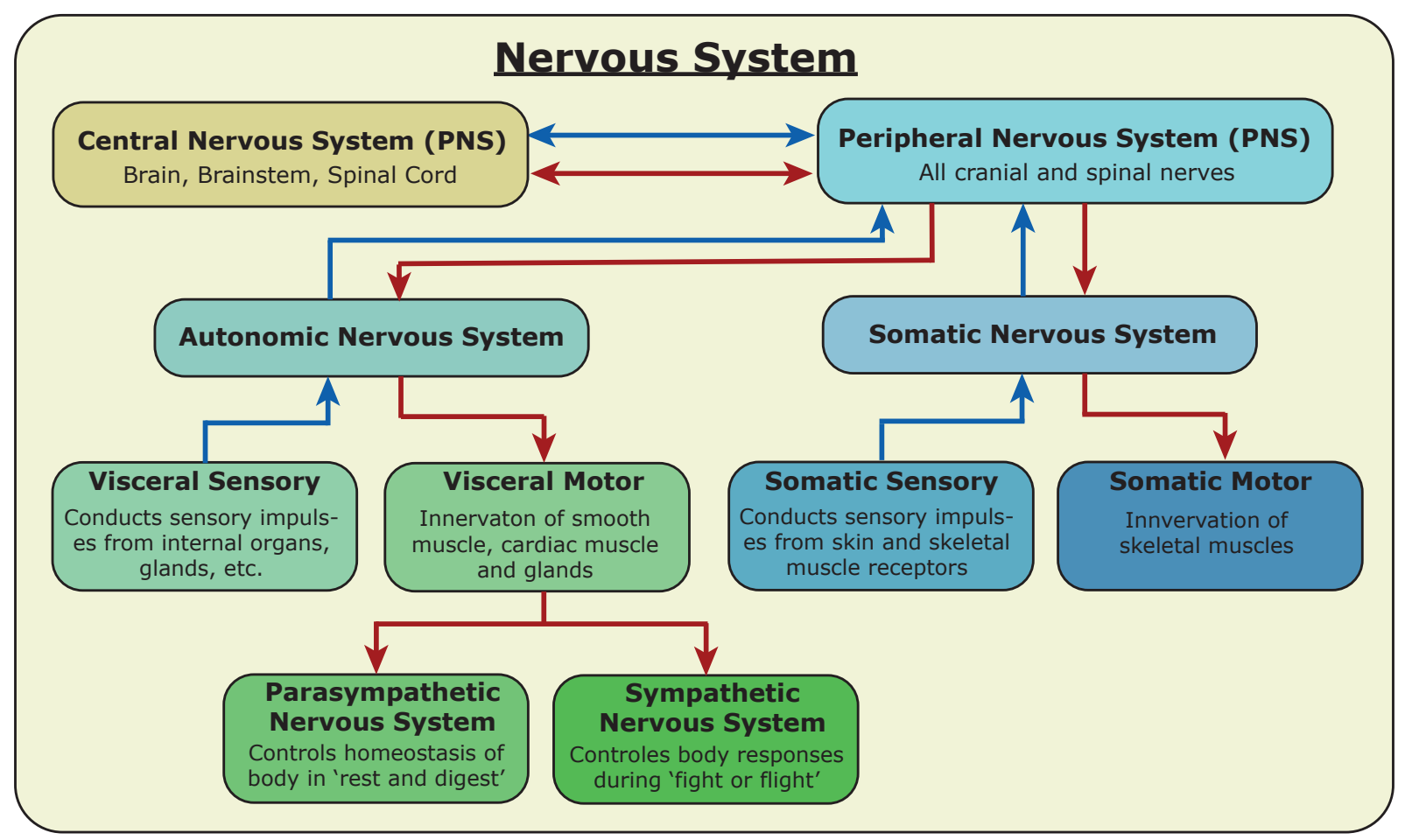

Figure 1.1: Nervous System - This block diagram shows the hierarchical organisation of the nervous system and the flow of both motor (red) and sensory (blue) information that travels through it.

too little or too much force to lift it. In a similar sense you will normally automatically grasp the screwdriver by its handle, as this is how you will use it, but if the handle is obscured by another object, grabbing it by the steel might seem more appropriate in this case. This shows how dynamic the movement plan can be and that a combination of visual information and memory is used (e.g. how you grabbed a similar object previously).

Once this movement plan is formed in a matter of a few hundred milliseconds, motor neurons in M1 that are linked to the skeletal muscles in the body start sending axon potentials down into the PNS. The PNS itself can be divided in an autonomic and somatic nervous system, respectively dealing with the subconscious and conscious control of the body. Note that both sections contain motor (efferent) and sensory (afferent) neurons. The somatic nervous system is the part we are focusing on in this thesis as it enables us to influence and interact voluntarily with the environment. Bundled in nerves, the axons of the motor neurons leave the spinal cord in so called spinal nerves, which start to branch off from there in a tree-like fashion. The arms have three main nerve branches: the median, ulnar and radial nerve. Once the motor axon reaches the endpoint muscle it connects to several muscle fibres, which constitute one motor 
unit. A contraction of the skeletal muscles creates angular momentum around the joints it connects causing a body part to move accordingly. This in turn triggers a cascade of sensory information: both the muscles and skin deforms due to the movement and possible contact with the environment and it is essential that this information is relayed back to the brain. Without sensory feedback you would not only be unable to sense when you are touching an object, but also proprioception (the sense of where your limbs are in space) would not be present, resulting in sloppy movements, that's why we will now further explore the information stream back to the CNS.

It is said humans have 5 different senses: vision, hearing, smell, taste and touch, but in reality there are many more specialised sensors. To name a few: balance, proprioception, temperature, acidity, blood oxygen levels and pain. These can be subdivided in even more specialised types and this wide range of physical and chemical sensors enables us to control our bodies and interact with our environment. Each of these sensors also have limits: while we can hear 10-25 kHz sound waves and see 400-700 nm wavelength light, we cannot detect e.g. ultrasonic sound nor ultraviolet light. Like so with our sense of touch, the mechanical sensory receptors in our skin are very specialised and limited to detect a certain stimulus.

Sensory receptors are structures in the human body that react to changes in their environment. These changes in the environment, also called 'stimuli', can either be internal or external and cause changes in neural firing rate in axons that travel through the PNS to the CNS, where they are processed and often followed up by a (voluntarily or involuntarily) motor response. The sensory receptors in the body are highly specialised for the type of stimulation and their location in the body. In general we can classify them under the following categories Marieb and Hoehn, 2007, Basbaum et al., 2009:

- Mechanoreceptors - respond to deformation of the adjacent tissue, due to pressure, touch, vibration and stretch.

- Chemoreceptors - respond to chemical changes in the adjacent solution (e.g. pH or CO2 changes) 
- Thermoreceptors - respond to thermal changes

- Photoreceptors - respond to light hitting them

- Nociceptors - respond to stimuli that can potentially harm (noci = harm) the body and will evoke a pain response when triggered. They are often linked with either one of the above receptors.

Since we are focusing on hand control, the mechanoreceptors are most interesting for us as these play a crucial role in somatosensory feedback. Somatosensory feedback integrates the information from multiple receptors embedded in the skin and muscles to provide a sense of touch (e.g. texture recognition), proprioception (sense of limp location in space) and haptic perception (object recognition).

Mechanoreceptors can be subdivided into four categories Ackerley and Kavounoudias, 2015. Marieb and Hoehn, 2007, Johansson and Flanagan, 2009, based on their type of ending (hairy skin vs glabrous), whether the axons are myeliniated or not, and on its adaptation properties to a sustained stimulus:

- Fast-adapting type I (FA1) - Meissner corpuscles

- Sensitive to: Dynamic skin deformations $(\sim 5-50 \mathrm{~Hz})$

- Highest density: Fingertips, Small receptive field

- Fast-adapting type II (FA2) - Pacinian corpuscles

- Sensitive to: Very high frequency skin deformations $(\sim 40-400 \mathrm{~Hz})$

- Highest density: equally distributed over the hand.

- Slow-adapting type I (SA1) - Merkel discs

- Sensitive to: Slow skin deformations $(<\sim 5 \mathrm{~Hz})$

- Highest density: Fingertips, Small receptive field

- Slow-adapting type II (SA2) - Ruffini endings 
- Sensitive to: Static force and skin stretching

- Highest density: equally distributed over the hand

Already in 1979 Johansson and Vallbo showed that the unit density of such mechanosensors in the human hand is incredibly high, ranging from $\sim 50$ sensors per $\mathrm{cm}^{2}$ in the palm to over $\sim 240$ receptors in the finger tips Johansson and Vallbo, 1979]. An average human hands has well over 10.000 mechanoreceptors, which allow us to discriminate very fine textures and interact very precisely with the environment. Of these, the FA1s are in the majority (43\%), followed by the SA1s (25\%), FA2s (13\%) and SA2s (19\%), but again the densities of these receptors are not equally distributed over the hand. For example, Meissner and Merkel endings are predominantly found in the finger tips [Johansson and Flanagan, 2009]. Also proprioception, the sense of where our limbs are in space, was initially solely contributed to muscle spindles, but it is now thought also cutaneous mechanoreceptors in the skin contribute to it, as the skin stretches widely during movement [Johansson and Flanagan, 2009].

The receptor's job is to encode the graded intensity of a stimulation into a burst of nerve impulses in a certain frequency. Once the receptor is stimulated above its threshold, it will cause a depolarisation big enough to transmit an action potential past its dendrites of the afferent nerve towards the soma. The greater the stimulus the higher the firing rate will be, though this transversion is not always fixed. While tonic receptors exhibit a sustained response with little to no adaptation, phasic receptors on the other hand adapt fast and only respond to changes in the stimulus strength.

On the way to the spinal cord the dendrites of the afferent neurons bundle together in nerves. Within a nerve, each neural fibre (motor axon or sensory dendrite) is encased in a myelin sheet, that enables fast transmission of the signal, and an encapsulating layer called the endoneurium. Subsequently, groups of endoneurium wrapped fibres are bundled bundled together in fascicles, wrapped in perineurium. The nerve itself consists of a fibrous sheet, called the epineurium that encloses several fascicles, blood vessels and lymphatic vessels, all separated by protective and insulating tissue. Note that most nerves contain both afferent and efferent fibres making them bidirectional information channels, but a general topography is maintained in which fibres that 
lie close to one another will have nearby end locations in the body.

Near the spinal cord the sensory and motor fibres seperate: sensory fibres enter the spinal cord through the dorsal root, while the motor fibres can be found in the ventral root (note that the information flows in opposite directions). In the spinal cord, the sensory fibres either transmit further up to the brain or they connect (either directly or more often indirectly through interneurons) to motor neurons where they can activate a (motor) reflex. The latter kicks in when, e.g., touching something hot, the intense activation of thermoreceptors and nociceptors sends up a signal to the spinal cord which inhibits antagonist and excites agonist muscle groups to move the hand away from the hot object. This process occurs very fast as it does not require processing in the brain. Note that the brain is able to suppress certain reflexes, therefore this process is more complex than a simple relay route.

From the point where the spinal cord enters the brain the signal gets distributed to different regions of the brain. One part heads to the cerebellum where amongst others, balance and posture control as well as motor learning and coordination is processed. Other projections go through the thalamus up to the somatosensory cortex (both primary S1, and secondary S2) [Marieb and Hoehn, 2007], where it turns sensation to perception. Activating specific mechanoreceptors in the skin does not only cause a sensation (i.e. of changes in the internal or external environment), but also a perception (i.e. an interpretation of the sensation). The perception of the environment does not only provide input to memory and decision making mechanisms, but it also feeds back to the motor cortex, returning at the start of our sensorimotor pathway. Note that the processing of sensory information is strongly modulated by attention and the behavioural context Romo and Salinas, 2001].

\subsection{Rehabilitation strategies}

Impairments to the sensorimotor pathways are most commonly caused by trauma, i.e. spinal cord or nerve damage. Brain trauma or neurodegenerative diseases also affect this pathway and can cause all kinds of motor and sensory deficits, but since the focus of this thesis is on 
peripheral nervous system interfaces we will focus on amputee patients that still have functional motor and sensory processing capabilities. These patients do not lose their ability to process the information cortically, instead it is the sensorimotor pathway that is interrupted somewhere along the way; the motor signals cannot reach the periphery while simultaneously no sensory information is transmitted towards the brain.

In this section we will first discuss the field of motor restoration using different neural interface strategies. With this information in mind, we then deal with sensory restoration as they often employ similar strategies.

\subsubsection{Neural interfaces for motor restoration}

The term 'neural interface' has already been mentioned a couple of times, but I have yet to give a proper definition of it. The National Institute of Neurological Disorder and Stroke describes it as follows:

'Neural interfaces are systems operating at the intersection of the nervous system and an internal or external device.'

In other words, it is a device that allow us to either extract information from the nervous system or influence the information flow that runs through it. These devices are usually electrodes in a specific shape and size to optimise the interaction with the neural tissue they are designed to interact with. These vary from non-invasive techniques (e.g. electrodes on the skin) to very invasive techniques (e.g. brain electrodes) each with their own trade-offs. These trade-offs mainly revolve around the selectivity of the interface versus the invasiveness, which involves the risk associated with using the device. I will run through the most commonly used neural interfaces ranging from non-invasive to very invasive.

\section{Electromyography}

Electromyography (EMG) is a technique that allows you to record the electrical activity of skeletal muscles. It is used for the evaluation of motor degenerative diseases and for the control 
of neuroprosthetic devices. By placing electrodes near muscle bodies, the changes in potential of the nearby muscle motor units can be picked up. While this usually involves surface EMG (sEMG) with electrodes on the skin surface, new techniques have been developed to implant EMG electrodes subcutaneously in or on the muscle itself, which reduces cross talk and allows recording from deeper muscle structures Morel et al., 2015, Farrell and Weir, 2008. EMG is currently the technique used for prostheses control in the state of art prosthetic devices like the iLimb (Touch Bionics) and Michelangelo Hand (Ottobock). This however, does not mean it is a new technique by any means. The Germany physics student Reinhold Reiter created the first myoelectric prosthesis in 1948 that amplified sEMG signals to drive motors. But it wasn't until 1960 that the technique gained more publicity, when the Russian scientist Alexander Kobrinski made the first clinically significant myoelectric prosthesis [Zuo and Olson, 2014].

While EMG has a big pro in ease of use apart from being non-invasive (in the case of sEMG), there are several factors that hold this technique back. First of all, the selectivity of the technique is limited. Without going invasive, it is difficult to isolate activity from single muscles, which makes simultaneous control of multiple DOF difficult. Second, sEMG is sensitive to changes in the conductivity of the skin, making re-calibration necessary when using it over an extended period of time. Not to mention that during movement the skin and the skeletal muscles are not in a fixed position relative to each other. While there are improvements made in signal acquisition as well as the data processing techniques that try to compensate for these changes Gijsberts et al., 2014], it is holding back this technique to give patients very dexterous control of their prosthetic devices.

\section{Brain Computer Interfaces}

In an attempt to not only increase the dexterity of prosthetic devices, but also to help patients with no muscle control at all (e.g. quadriplegic patients), brain computer interfaces (BCIs) came into interest. Instead of recording the activity of many muscle units (and their attached motor neurons) like in EMG, brain interfaces record activity directly from the neurons in the brain. With the risk of being highly invasive, more information is gained. 
There are many different BCI interface types each with their own pros and cons. For example, electrocorticography (ECoG) involves laying a grid of electrodes on the brain's surface and recording from many neurons at the same time. It is mainly used in the context of epilepsy surgery. Wile it is also possible to decode hand movement intention with it, it suffers from low spatial resolution in the same way that EMG does [Spüler et al., 2014]. On the other hand, floating micro arrays (FMA's) and Utah arrays employ a different tactic by penetrating the cortex and spacing the electrodes very closely together. This allows the recording of single neuron activity in various brain areas and significantly ramps up the decoding capability. The developments of intracortical recordings have come a long way since; Collinger et al., 2013] implanted two 96-channel intracortical microelectrodes in the motor cortex of a 52-year-old tetraplegic patient and after 13 weeks of training she was able to operate a 7 DOF robotic arm.

Evaluating these experiments we can point out a couple of challenges. First, the neural coding in the brain is very complicated compared to the simple coding in the PNS, where a higher firing rate simply means a stronger contraction of the muscle. However, as discussed in the previous section, the motor areas in the brain do not only convey direct muscle control, but also encode action, goal and motivation. This complicates training classifiers (also called decoders) for prosthetic control, as the neural activity can reflect, for example, a goal instead of moving a limb in a specific angle. For prosthetic use, decoding from M1 seems most efficient Schaffelhofer et al., 2015, Carpaneto et al., 2012, , but from pre-motor areas one can also derive a lot of information about the movement intention Menz et al., 2015, Carpaneto et al., 2012, Townsend et al., 2008.

Another challenge is choosing the right type of classifier and the amount of training data. While complex neural network decoders might perform well in a certain situation, Kalman filters might excel in another [Sussillo et al., 2015, Welch and Bishop, 1995, Menz et al., 2015]. The "optimal" decoder in that sense is strongly dependant on the task type and the signal type, though in practice many different decoders function reasonably well [Koyama et al., 2010]. The improvements between using a fairly complicated decoder versus, e.g., a simple linear supportvector-machine (SVM) decoder, is sometimes limited to a couple percent. Which gives rise to the idea that perhaps we should not focus too much attention on optimising the decoder, but 
instead on setting up a decent decoder and training the brain in using it [Scherberger, 2009]. Ganguly and Carmena, 2009 showed this by first training a decoder on a set of neurons during a center-out reaching task and after training, shuffling the decoder weights and keeping them fixed throughout the experiment. What they saw was that the brain was able to re-adjust to this shuffled decoder after about 10 sessions, showing the neural code is plastic enough to learn to work with the means given.

Another aspect to keep in mind is that these decoders are not interface specific: a griptype decoder using M1 neurons might also work if you feed it EMG data. In general the more (meaningful) information the decoder has access to, let that be neurons or EMG channels, the better the performance is Carpaneto et al., 2011. In addition, the more stable the signals are, the better we are able to work with them. This brings us to a big limitation of (invasive) neural interfaces: the lifetime of the electrodes. For all brain interfaces, the brain tissue will reject the foreign material at some point in time, by embedding it in scar tissue. Biocompatiblity limits are 1-5 years, which is enough for experiments, but not for long term restoration in patients Barrese et al., 2013. Also, it often requires months of training to learn to work with a neural decoder as the brain has to rewire itself to learn this new movement strategy. With this in mind we see that the biocompatibility issue in all these brain interfaces limits the practical usability considerably. Some BCI users even experience so called 'BCI illiteracy', they are not able to learn to use their BCI well enough for effective control [Choi et al., 2017].

\section{Targeted Muscle Reinnervation}

As discussed before, EMG struggles with crosstalk between muscle groups because they are closely located. Another limitation is that depending on where an amputation took place, you might not have access to the lower arm muscles that are normally used to control the hand. Targeted muscle reinnervation (TMR) is a technique that overcomes these problems Roche et al., 2014. Kuiken et al., 1995 showed that you can take a nerve and implant it into a different muscle than it was originally heading to, and the nerve can activate this new muscle group. In case of arm amputee patients, you can take the nerves that lead to the hand muscles 
and implant them into an intact proximal muscle (often the chest muscle because of its size and the convenience of placing EMG electrodes on them). After an extensive recovery period the patient can for example flex his/her wrist and the chest muscle will contract at a specific location instead. After mapping different movements and consequently placing EMG electrodes on these locations on the skin, the patient is able to control the prosthetic device by 'thinking' of moving his/her original hand. While this seems an ideal solution, in reality the targeted muscle is not always successfully reinnervated. Also the other EMG problems regarding skin conductivity are still present.

The neural interface strategies that have been discussed in this section are only a selection of all the available neural interfaces, but they give an overview of the wide range in selectivity and invasivity that they span. Each have their own strengths and weaknesses, which makes them suitable for a specific application. Regarding motor restoration, one generally favours a higher selectivity as this opens up possibilities to interface much more accurate with the nervous system. This also holds true for somatosensory restoration, which is discussed next.

\subsubsection{Sensory restoration}

\section{Organ Stimulation}

When trying to restore a sensory percept, it is often the easiest to interface as close to the origin (or sensor) of that percept. A major contributing factor to this is that neural encoding in the brain is highly advanced and still not completely understood. For example with vision, we know that the axons from the retina innervate V1 first, but not even the mapping at this site is fully understood and beyond V1, the specialisation and abstraction of the signal increases exponentially. Therefore, restoring sensory percepts has a higher chance if the brain is able to do the processing and stimulation occurs closer to the origin of the signal (the retina in this case) Hadjinicolaou et al., 2015. The most successful and widely known sensory restoration by organ stimulation is the cochlear implant. In October 1982, Graham Carrick (from Melbourne) made history when electrodes were implanted in his cochlear and he could hear again for the 
first time in 17 years. At the end of 2010 there were already more than 200.000 people walking around with a cochlear implant and this number has only increased since then Behan et al., 2017 .

Despite this success story in restoring hearing, the act of restoring the sense of touch is more complicated than that. As the sense of touch does not have a single dedicated organ, it makes it more difficult to make a selective prosthetic device for it. But it does not mean we cannot make use of the same principles of letting the brain handle the processing. Johansson and Westling, 1984 already hinted at the underlying non-cortical mechanisms in slip detection and Pruszynski and Johansson, 2014 recently showed that sensory processing of edge detection in touch is occurring at the PNS level. These are indicators that the strategy of interfacing as close to the sensory end points increases your chances of restoring the percept and it will restore a more natural precept to the patient. But before we delve deeper into that, I first want to discuss a field of sensory restoration that takes the brain plasticity to an even higher level.

\subsubsection{Sensory substitution}

As [Paul and Kercel, 2003] nicely stated:

'Sensory substitution is only possible because of brain plasticity.'

'Plasticity', the brain's ability to adapt its neural code, plays a critical role in interacting with the environment and solving problems [Kiper et al., 2007]. It is also something that can be manually induced as Jackson et al., 2006a showed that brain coding can be altered when artificially stimulating a different region in co-junction.

We can venture on this extraordinary ability for sensory restoration. Instead of trying to repair the original organ or stimulating the brain, one can also approach another strategy, namely to replace the lost sensory modality by a different one. An interesting example of this is the BrainPort, which is used to substitute vision [Danilov and Tyler, 2005]. As discussed before, stimulating the retina directly involves a lot of technical difficulties, among others having only a small surface to work with. What Danilov et al. have done instead is to place an electrode array in the mouth and stimulate the tongue instead. The other techniques up to this stage 
are the same: i.e. record a video signal, apply imaging techniques like edge detection on it, downscale the signal resolution so that it matches that of the electrode grid. However, with BrainPort the tongue is electrically stimulated, instead of the retina. The authors have shown that shape recognition is possible and this method has the advantage that it only requires hardware and no invasive surgery.

The downside with all these techniques is that there is a learning curve involved that is not (as much) present when completely restoring the natural sensation. But when it is not possible to restore or mimic the original sensory modality, due to biological and/or technical limitations or when it is not practical or too risky to do so, sensory substitution could provide the means to give a patient some sense of autonomy back.

\subsubsection{Targeted sensory reinnervation}

We have already discussed targeted muscle reinnervation, but only covered the motor aspect. As the nerves are bidirectional channels, aside from reinnervating the motor fascicles one can also redirect sensory fascicles to a new location. Hebert et al., 2014 did exactly this, because they not only coapted the motor fascicles of the median and ulnar nerve to the biceps and the brachialis muscles, respectively, but they also identified high sensory fibre fascicles through intrafascicular dissection in both the median and ulnar nerve which they innervated close to the skin in the intero-costobrachial cutaneous nerve and axillary nerve. The reinervation of the sensory fascicles caused a skin map of the hand being formed on the residual limb. When pressure sensors of a robotic gripper where linked to a tactor that applied pressure to this piece of skin, it felt as if the patients hand was touched. As with targeted muscle reinnervation, this technique is heavily dependent on the surgery techniques and the hardware's ability to stimulate the new piece of skin in a way that feels familiar. Another aspect to keep in mind is that the newly innervated piece of skin will not be as densely packed with mechano-receptors as the intact hand was, therefore restoring the original spatial sensory resolution is physically not possible. 


\section{Cortical micro stimulation}

Brain interfacing for somatosensory restoration employs a similar strategy to cortical motor decoding: namely interfacing in the brain regions where the information type is processed. The difference is that for motor decoding one wants to extract information, whereas for somatosensory restoration one has to inject information in the system. Just as with the motor cortex, the sensory cortex has a topographical representation of the body, where different regions of the body are processed in localised regions Marieb and Hoehn, 2007]. Medina et al. and Bensmaia et al. showed that it is possible to evoke sensory percepts using intracortical microstimulation (ICMS) in the sensory cortex Medina et al., 2012, Bensmaia and Miller, 2014, Tabot et al., 2015]. They mapped the sensory cortex by tactilely stimulating the hand and simultaneously recording neuronal activity from the sensory cortex with cortical arrays. By consequently stimulating these locations with electrical pulses they were able to evoke a sensory percept in the hand. Modifying the stimulation parameters allowed for changing the percept and intensity of these artificially evoked sensations [Romo et al., 1998, Tabot et al., 2013.

While they were successful in evoking sensations, two issues have to be pointed out with this strategy. First, the cortical mapping of the body does not hold up anymore at subregions of the hand, so fine mapping of the individual fingers is not straightforward. A bigger issue is that it is questionable how natural the evoked sensation feels. A big problem with ICMS is that it interfaces directly at the site where the processing happens. Injecting current there evokes massive synchronised activity, which is unlikely to resemble how the brain would have processed the signal. Aside from that, our run through the sensorimotor pathway showed that $\mathrm{S} 1$ is not the only region that processes sensory information. By stimulating the cortex directly one leaves these regions out of the loop. While this method might see limited usability with for example quadriplegic patients, for amputee patients a more promising strategy could be to interface at the PNS level to evoke sensory percepts. 


\subsubsection{Nerve interfaces}

Whether dealing with motor decoding for prosthetic control or electrical stimulation for somatosensory feedback with amputee patients, ideally we would like to interface directly with the nerves, preferably as close to the end point as possible. For motor decoding we have the advantage that we are recording directly muscle related activity and the more distal we are, the more selective it will be for the hand. With respect to somatosensory stimulation we can use the same type of interface to stimulate the nerves and, since it is following the original pathway up to the brain, it will hopefully feel more natural as well.

New manufacturing techniques allow the fabrication of such interfaces. And like cortical neural interfaces we can again separate them based on invasivity vs selectivity. By invasivity we refer to the severity (and thus risk) of applying this interface on/in the body. The selectivity is a measure of how well the system is able to record or stimulate a chosen set of axons Durand et al., 2005. I will present 4 different types of PNS interfaces: cuff, LIFEs, TIMEs and lastly Sieve electrodes [Micera et al., 2010]. I will also briefly touch on CNS interfaces as USEAs for PNS interfacing.

Single channel needle electrode recordings formed the foundation of understanding the neural language in the PNS, which both for motor and sensory encoding translates a higher muscle contraction or sensory stimuli into a higher firing rate Rice et al., 2015, Romo and Salinas, 2003. We do have to keep in mind that, like many biological processes, the actual coding is far more complex. A study from Pruszynski and Johansson, 2014 showed that sensory percepts like edge detection are already encoded very distal in the PNS system. Though it still holds that the PNS encoding is more straightforward than the CNS encoding. These needle electrodes allow for a high degree of control with respect to which fascicle to record from. However, this method is limited to acute and static experiments, since these are not designed for long term recording or experiments that involve limb movements.

In an attempt to move to an implantable solution, cuff electrodes have been developed Sahin and Durand, 1997]. Like the name suggests, they are arrays of a small number of electrodes 
(typically 4-8) orientated in a ring pattern on a cuff that is slid around the nerve. As cuff electrodes are extraneural, they score low on the invasiveness ranking in respect to other PNS interfaces, which require puncturing of the nerve, but still a percutaneous surgery is required [Raspopovic et al., 2010]. A study from [Tan et al., 2015] show that cuff electrodes can remain selective for somatosensory stimulation up to 2 years in the human body. This comes at the price that the selectivity is quite low, which makes recording and stimulating individual fascicles challenging.

To step up the selectivity we arrive at the intrafascicular electrodes, which actually penetrate the nerve Boretius et al., 2010]. Thin-film Longitudinal Intrafascicular Electrodes (tf-LIFE) do this allong the length of the nerve and are implanted inside a fascicle. This gives excellent selectivity for this particular fascicle, but they capture only a fraction of the information travelling through the nerve. Studies have shown that it is also possible to detect neural information and decode grasping information using these intrafascicular interfaces Micera et al., 2010, Micera et al., 2011, Dhillon et al., 2005, Dhillon and Horch, 2005, Rossini et al., 2010].

On the other end of the spectrum are SIEVE electrodes, which are very invasive. They require cutting of the nerve and inserting a sieve like electrode grid in between the ends. By stimulating growth inside the sieve it is hoped that the axons will regenerate through the pores which then allows recording or stimulating with a high selectivity [Dario et al., 1998]. Unfortunately, the growing back part is still a big challenge, since neural tissue is notorious for its poor regeneration capabilities.

Clark et al. took a different approach and implanted Utah Slanted Electrode Arrays (USEAs) in the peripheral nerves Clark et al., 2011, Clark et al., 2014. These high count (100 channel) electrode arrays had previously been used in the CNS (both brain and spinal cord) experiments. While they showed that they were able to detect neural spiking related to the phantom limb movements and stimulate the nerve to evoke sensory percepts, the amount of recorded information was limited. This is due the fact that despite the high electrode count, one can only measure a fraction of the nerve's information, since with an average electrode length of about $1 \mathrm{~mm}$ USEAs puncture the epineurium, and therefore have access only to the outer fascicles. 
The interface that strikes a middle ground between these interfaces in terms of invasivity and selectivity is the Transverse Intrafascicular Multichannel Electrode (TIME). Testing, recording and stimulation with this interface forms the foundation of this thesis. These thin-film electrode arrays penetrate the nerve transversally and allow for recording and stimulating of the individual fascicles it penetrates. Recently it was shown that stimulating the median and ulnar nerves successfully allowed an amputee patient to distinguish different textures and surfaces from another Raspopovic et al., 2014, Oddo et al., 2016].

In a study by Badia et al., 2011b the selectivity of Cuff, LIFE and TIME electrodes was compared. Here they showed that all are able to evoke neural activity in the nerve with electrical stimulation, however, while cuff electrodes mostly excite superficial fascicles, TIMEs are able to excite deeper fascicles as well. This in turn meant that the researchers were more successfully able to target individual muscle groups with the TIMEs. LIFE electrodes, on the other hand, are very selective, but only for a single fascicle. This means only a single muscle group could be targeted per LIFE. Because the epineurium is already penetrated, the LIFE and TIME do have significantly lower stimulation thresholds than cuff electrodes. One should note that the increase in selectivity comes at the price of a shorter lifetime. As mentioned before, cuff electrodes have been proven to last up to two years in the human body Tan et al., 2015], while the longest TIME implantations in humans have only lasted about a single month Raspopovic et al., 2014, Oddo et al., 2016

\subsection{Motivation and objectives of this thesis}

With this introduction in the field of bidirectional neuroprosthetics I hope to have shown that there are many different strategies for the restoration of body control and somatosensory sensation, each with their own separate strengths and weaknesses. In this thesis I am focusing on technique developments that will benefit amputee patients who have (partially) lost an arm, severely impeding them in their ability to interact with and manipulate the environment. On paper the TIME interface seems like a promising option for this group of patients, compared to the other available options. sEMG for example is an user friendly applicable technique that 
does not require invasive surgery, but we are reaching the limits of this technique in regards to multiple DOF control. Also sensory feedback is completely missing with this interface, something that has to be included if the aim is to restore a person's hand function. TMR does allow for bidirectional control of a prosthetic device, but for prosthetic control one is still tied to the EMG interface with all its limits. Cortical implants for both neuronal recording and ICMS are debatable too invasive measure for amputee patients and are more suited for people who have lost spinal nerve activity. And while multiple simultaneous DOF prosthetic control has been achieved, it is questionable whether ICMS of the sensory cortex is able to provide both accurate and natural feeling somatosensation. The TIME interface, on the other hand, could not only provide a way to tap into the nerve motor information stream, but also makes it possible to stimulate the PNS directly, leaving the higher level somatosensation processing stream intact, which could provide a more natural feeling of touch.

This thesis aims to provide answers to how well TIME interfaces perform with regard to the motor decoding for prosthetic control as well as the provision of somatosensory feedback. For this thesis, I implanted TIME electrodes in the ulnar in a rhesus macaque monkey. The animal was trained for two specific task designs; a delayed grasping task that investigates the motor decoding aspect, and a somato-sensory discrimination task that investigates somatosensory feedback. Lastly, by monitoring the TIME electrodes performance over time I explored the biocompatibility of these neural interfaces. 



\section{Chapter 2}

\section{Materials and Methods}

The methods section is divided in three sections.

In the first section 'Neural Interface' we will go through the specifications of the TIME arrays, the neural interface we use in all the experiments, as well as the methodology of the surgical procedures for both terminal and long term implantations. The TIME arrays are fabricated by Matthias Müller at the Institut für Mikrosystemtechnik (IMTEK) at the University of Freiburg. The surgeries are performed by Prof. Dr. Hansjörg Scherberger.

The second section describes the motor decoding task, i.e. the task design, the experimental setup, the data acquisition and data analysis. This motor decoding task has been designed by Stefan Schaffelhofer and was rebuild and executed by me. The data organisational structure is an adaption of his work, while the analysis code is written by myself.

The last section goes over the challenges that we had in designing the somatosensory discrimination task and follows up with a detailed description of the experimental setup and data analysis techniques. The supplements (see Section A.1) go deeper into the validation of the vibration glove motors. In the design of the somatosensory discrimination task I had the help of my Master students Laura Jens and Luis Ángel Pardo Sánchez who assisted in validating and testing the vibration motors, and with animal training.

Animal care and all experimental procedures were conducted in accordance with German and European law and were in agreement with the Guidelines for the Care and Use of Mammals in Neuroscience and Behavioural Research Council, 2003. 


\section{$2.1 \quad$ Neural interface}

\subsubsection{Transverse Intrafascicular Multichannel Electrodes (TIME)}

\section{Electrode design}

Recent developments in electrode manufacturing technology allow the fabrication of thin film electrode arrays that can be implanted in the nerve. Such electrodes allow multi-channel recording and electrical stimulation of individual fascicles in the nerve. The Transverse Intrafascicular Multichannel Electrode (TIME) is a novel electrode that has been developed at IMTEK (University of Freiburg) is tested in the NEBIAS project Boretius et al., 2010, Badia et al., 2011a]. The TIME electrode is made as a thin strip of polymide, with a width of $200 \mu \mathrm{m}$, that is narrowed at the idle part to $100 \mu \mathrm{m}$ in the center (see Figure 2.1). The strip has a $90^{\circ}$ angle between the ribbon part and the insertion part. The TIME electrode contains 16 active sites $(8$ per side), coated with iridium oxide (IrOx; $900 \mathrm{~nm}$ thick, $90 \mu \mathrm{m}$ in diameter) with an additional ground electrode per side.

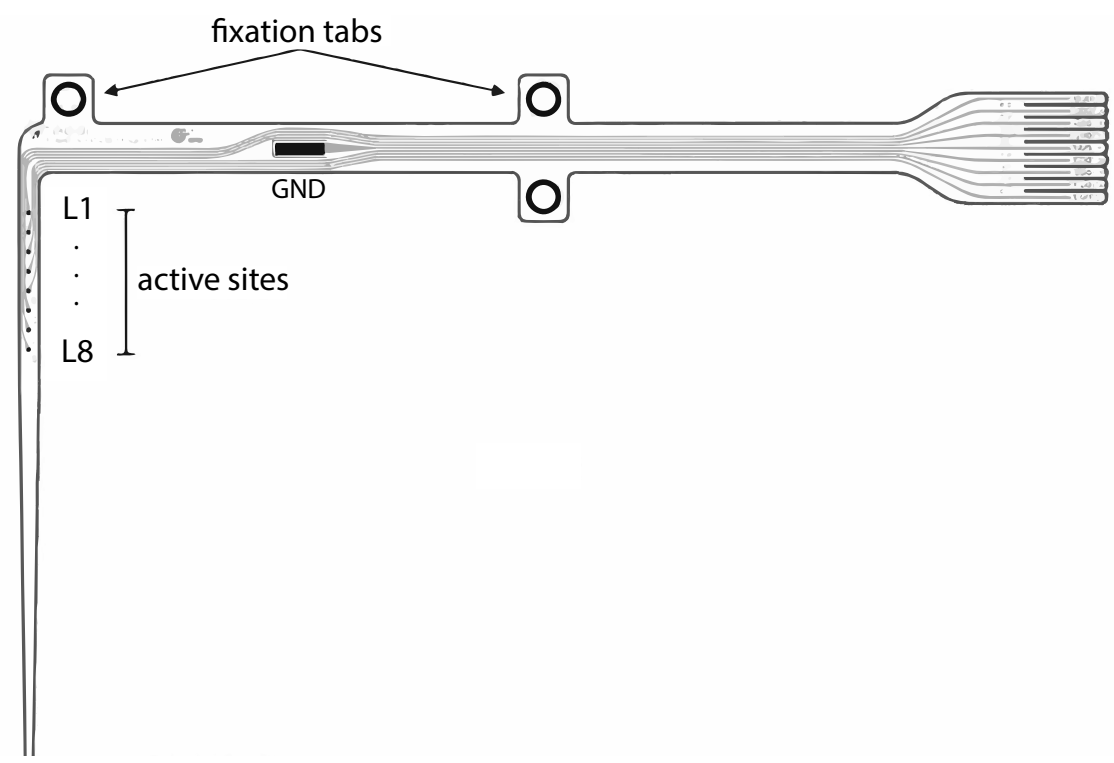

Figure 2.1: Schematic design of the TIME electrode - Internal wiring is indicated by the grey lines. Note that only the left side of the TIME is visible, right side is an identical mirror image. Both sides are produced out of a single sheet of film that is folded at the distal end of the active side strip. 
The internal cables are helically wound and are sealed in a polyesterimide (PEI) running through a silicone tubing, ending in a 16-pin Omnetics connector (see Figure 2.2). As the connector has fewer pins than the TIME has active sites, only 7 active sides per side are utilised (the most proximal active sites (L8 and R8) are not connected). Per side also one of the active sites functions as reference, which leaves us with a total of 12 channels available for stimulation and recording. The average impedance of the active sites lies around $5 k \Omega$ and that of the ground electrodes lies around $700 \Omega$ (both tested in saline solution at $1 \mathrm{kHz}$ ).

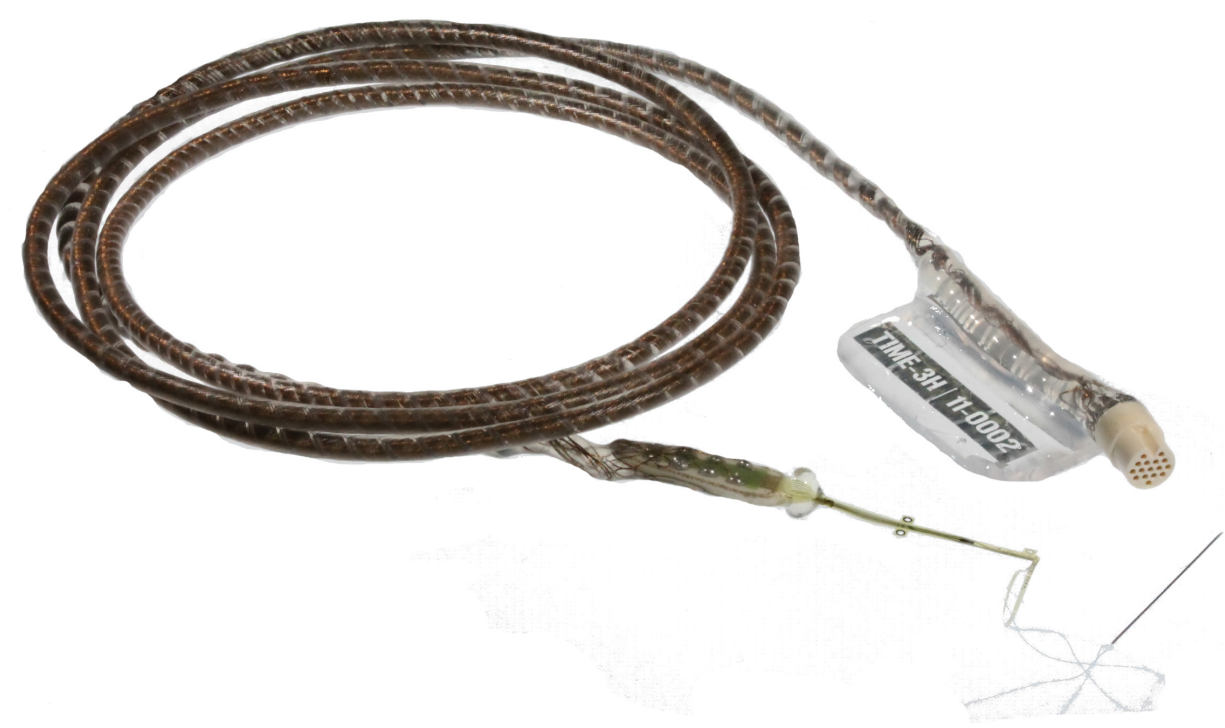

Figure 2.2: Overview of complete TIME array - The TIME array is connected to $50 \mathrm{~cm}$ of spiralled wire, coated in silicon and ends in a 16-pin Omnetics connector. A thin needle, which is used to pierce the nerve and thread the array through, is attached to the end of the TIME array with a suture wire.

To prevent electrode failure due to electrochemical corrosion while electrically stimulating, one should not exceed the maximum allowed charge injection, $Q_{\max }$, defined as the charge of a single phase in a stimulation pulse.

$$
Q_{\text {max }}=t_{\text {phase }} * I_{\text {phase }}
$$

With $t_{\text {phase }}$ being the phase width of a single pulse in seconds and $I_{\text {phase }}$ the amplitude of that phase in ampere. The active sites of the TIME arrays are rated for a maximal injected charge of $120 \mathrm{nC}$ Boretius, 2012.

The polymide thin film of the TIME is too fragile and not stiff enough to penetrate the 
epineurium by itself. The nerve is therefore first punctured with a needle to create a gateway for the TIME. With a thin suture thread that connects the TIME with the needle, it can then be pulled through the nerve. After confirmation with a surgical microscope that (most) active sites are located inside the nerve, the TIME is sutured to the nerve's epineurium using the fixation tabs (see Figure 2.1.

\subsubsection{Implantation techniques}

\section{Acute Experiments}

As implantations of TIME electrodes had not been performed on rhesus macaques there was limited knowledge about the feasibility of the surgical procedures. For this reason two acute experiments have been performed for surgical exploration of the median and ulnar nerve. The goals of these experiments were to explore:

- The approach the median and ulnar nerves

- The placement of cuff and TIME arrays on/in the nerve

- The tunnelling of the electrode cables under the skin

- The electrical stimulation of the nerve

- The recording capabilities of the electrodes

\section{Short-term experiment}

The next step was to perform a short-term experiment. During the two weeks the TIMEs stayed in we had the chance to investigate the effects of the implantation on an awake animal. Especially the tolerance of the subcutaneous cabling was something unexplored till then. Aside from this, it also gives me the answer whether the electromagnetic field from the hand tracking system would influence the recording of the nerve activity. Lastly the reaction of the awake animal to the electrical stimulation was tested. 
After a recovery period of a week, we attempted to record neural activity from the electrodes. While the monkey was not trained to perform a timed grasping task, it could grasp a handle when it was presented to it, which was sufficient to evoke a repetitive movement of the arm and hand. Our main interest from these recordings was to investigate whether the electromagnetic field generated by the hand tracking system would introduce additional noise to the recording. As described in detail in Section 2.3.2 the hand tracking system generates an alternating electromagnetic field which in turn generate a current in the coils that are connected to the hand. Based on the electric current strength, the position of the coils in space can be calculated. As the wire of the TIME electrode array is also coiled and it will move in the same electromagnetic field (during the final task design), it is not unimaginable that it would pick up noise from magnetic field generated by the hand tracking system. By comparing recording samples, which were taken while the electromagnetic field was either turned on versus recordings without any electromagnetic field, I could decide whether or not it is feasible to use the hand tracking system for the final task paradigm.

\subsubsection{Surgical procedure - Long-term experiments}

Once the monkey was trained for both the motor decoding and somatosensory-discrimination task (see respectively Sections 2.3 and 2.4), it was ready for a long-term implantation of the TIME arrays. Due to space constraints in the arm we were limited by a single TIME in each the median and the ulnar nerve. In total two long-term implantations have occurred. First the left arm was implanted and a year later, after the first arrays failed and both tasks were trained for the other arm, the right arm was implanted using the same procedure.

\subsubsection{Deplantation of TIME arrays}

Once both TIME arrays were not able to evoke a muscle response with electrical stimulation at the maximum stimulation settings allowed by the CereStim96 stimulator (biphasic pulse train, 60 pulses, frequency: $150 \mathrm{~Hz}$, amplitude: $210 \mu \mathrm{A}$, phase width: $500 \mu \mathrm{s}$, interphase: $53 \mu \mathrm{s})$, it 
was decided to surgically remove the arrays and subcutaneous cable.

After the second implantation the animal was sacrificed and perfused in paraffin so histology could occur on the implanted nerves. After the deplantation of the TIMEs, segments of the nerves with the TIME thin-film attached, were prepared by Prof. Xavier Navarro's team at the Department of Cellular Biology, Physiology and Immunology at the Institut de Neurociències (INc) of the Universitat Autònoma de Barcelona (UAB).

\subsection{Monitoring electrode longevity}

To monitor the electrode longevity two parameters are measured over time:

- the impedance of the electrode contact sites

- the stimulation threshold of each electrode channel

The impedance of the electrode array contact sites gives us an indication if a technical failure of the TIME electrode occurred, i.e. breakage of the cable or thin film or the loss of contact site coating. The impedance was measured with the CereStim96 stimulator (Blackrock Microsystems) with a custom developed MATLAB GUI that interacts with the device (see Figure: 2.14). An electrode channel was marked as 'functional' if the impedance was lower than $200 \mathrm{k} \Omega$. It is very well possible that the impedance of a channel could have exceeded this level and still be functioning, but as this was the highest impedance the stimulator could measure, we could not further distinguish it from a broken channel with the means at hand. However, since the impedance of brand new contact sites lie around the $5 \mathrm{k} \Omega$, an increase of a factor 40 does indicate something is amiss.

Apart from technical failure of the electrode array, also 'biological' failures could occur. The formation of scar tissue around the thin film, or slippage of the array in our even out of the nerve are potential factors that could negatively influence the recording and/or stimulating capabilities of the TIMEs. By periodically investigating which stimulating charge was necessary to evoke neural activity in the nerve, we have another measure to track the electrode condition. 
This stimulation threshold was defined as the minimum charge of a single phase in a biphasic pulse train (frequency: $150 \mathrm{~Hz}, 60$ pulses, amplitude: 10-210 $\mu \mathrm{A}$, interphase time: $53 \mu \mathrm{s}$ ) that evoked a muscle twitch in the hand. With the impedances and the stimulation threshold combined we can conclude that if a channel has low impedance levels, but is not able to evoke motor activity, then we know there is either too much scarring around the electrode or that it slipped either out of the nerve or in between fascicles.

\subsection{Motor Decoding task}

\subsubsection{Basic procedures}

One purpose bred female rhesus macaque $(7,8 \mathrm{~kg})$ was trained to perform a delayed grasping task on a wide variety of objects. These objects were presented on a rotating turn table. After the object were presented and the go-cue was given, the monkey had to grab and lift the object for $500 \mathrm{~ms}$ to receive a liquid reward.

After the training phase, the animal was implanted with a TIME electrode in both the median and ulnar nerve in the upper arm. These were then used to record the neural activity from the median and ulnar nerve. Simultaneously the hand kinematics were tracked with a hand tracking glove.

\subsubsection{Experimental setup}

During the experiments the animal was seated in a specialised animal chair with the head fixated in forward facing position. The arm not used for the task (i.e. the non-grasping hand) was placed, in a natural resting position, in a tube to prevent interference with the experiment. The animal could initiate a trial by placing its unrestricted grasping hand on a capacitive switch (referred to as handrest button). This switch operates both as sign for the animal to show compliance in performing the task as well as a safety switch, as the turn table cannot not move if the button is not pressed. 
The turntable was located in front of the animal with the objects just below shoulder height at $\sim 15 \mathrm{~cm}$ away from resting position (see Figure 2.3). The turntable box contained 1 out of 7 exchangeable turn tables that allowed the grasping of 36 different objects. 6 out of 7 tables contained objects from the same group (i.e. circles, cubes, horizontal bars, horizontal cylinder, vertical cylinder or rings) each of different sizes (see Table 2.1). To reduce the effect of shape size on the effort to lift the object, all objects had an uniform weight of $120 \mathrm{~g}$. A single 'mixed turntable' contained a single object from each group: sphere (15mm), horizontal cylinder $(30 \mathrm{~mm})$ cube $(30 \mathrm{~mm})$, vertical cylinder $(30 \mathrm{~mm})$, horizontal bar $(30 \mathrm{~mm})$ and ring $(50 \mathrm{~mm})$. While the animal was trained to operate each table, recordings were focused on the mixed turntable as it evoked the most varied grasping kinematics.

Table 2.1: Turn Table Objects - All units are displayed in mm

\begin{tabular}{|l|l|l|l|l|l|}
\hline Ring & Cube & Ball & h. Cylinder & Bar & v. Cylinder \\
\hline outer $\oslash(\mathrm{mm})$ & l,w,h $(\mathrm{mm})$ & $\oslash(\mathrm{mm})$ & $\oslash(\mathrm{mm})$ & depth $(\mathrm{mm})$ & $\oslash(\mathrm{mm})$ \\
10 & 15 & 15 & 15 & 15 & 15 \\
20 & 20 & 20 & 20 & 20 & 20 \\
25 & 25 & 25 & 25 & 25 & 25 \\
40 & 30 & 30 & 30 & 30 & 30 \\
50 & 35 & 35 & 35 & 35 & 35 \\
60 & 40 & 40 & 40 & 40 & 40 \\
\hline & & & length: 140 & length: 140 & length: 140 \\
& & & & height: 50 & \\
& & & & depth: 15 & \\
\hline
\end{tabular}

The turntable itself was belt driven by a step motor controlled through a LabVIEW virtual instrument (VI). The reason why the turn table is belt driven, is that the hand tracking system (see Section 2.3.2), loses it's accuracy when there is a lot of metal near the tracking system. Moving the belt (and other electronics) as far from the turn table itself greatly improves this. Apart from motor control, this VI also controls the rest of the behavioural paradigm, including lights, triggers, rewards as well monitors the performance. This data is all synchronised with the behavioural parameters and stored in the recording system. An overview of the VI main page is visible in Figure 2.4 a data flow diagram with all the components in the setup can be found in Figure 2.5 . 

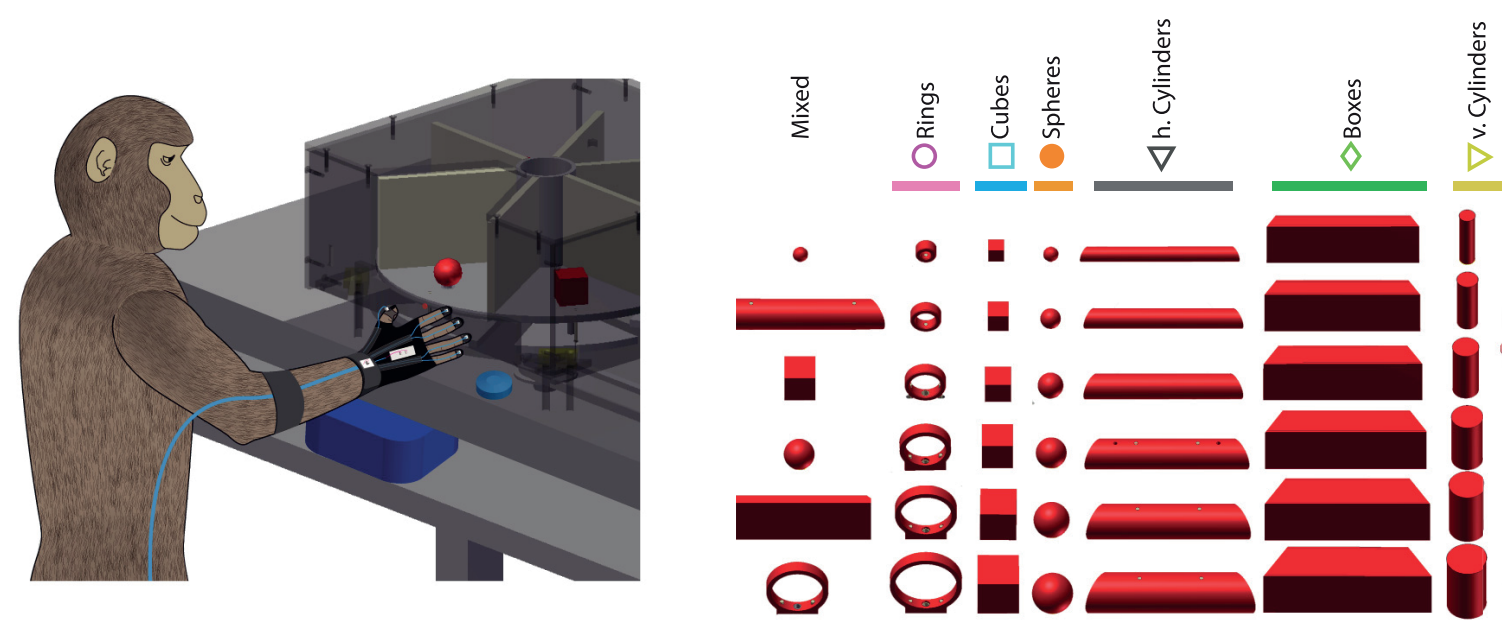

Figure 2.3: Motor Decoding Task - The animal is situated in front of a turntable (left) on which 36 different objects can be presented, which are distributed over 6 different turn table groups (right), excluding a mixed turntable with a single object front each group. The monkey is trained to initiate a trial by pressing the hand rest button and, after the go-cue, to grab and lift the presented object for $500 \mathrm{~ms}$ to receive a liquid reward (grape juice). Figure taken from Schaffelhofer et al., 2015].

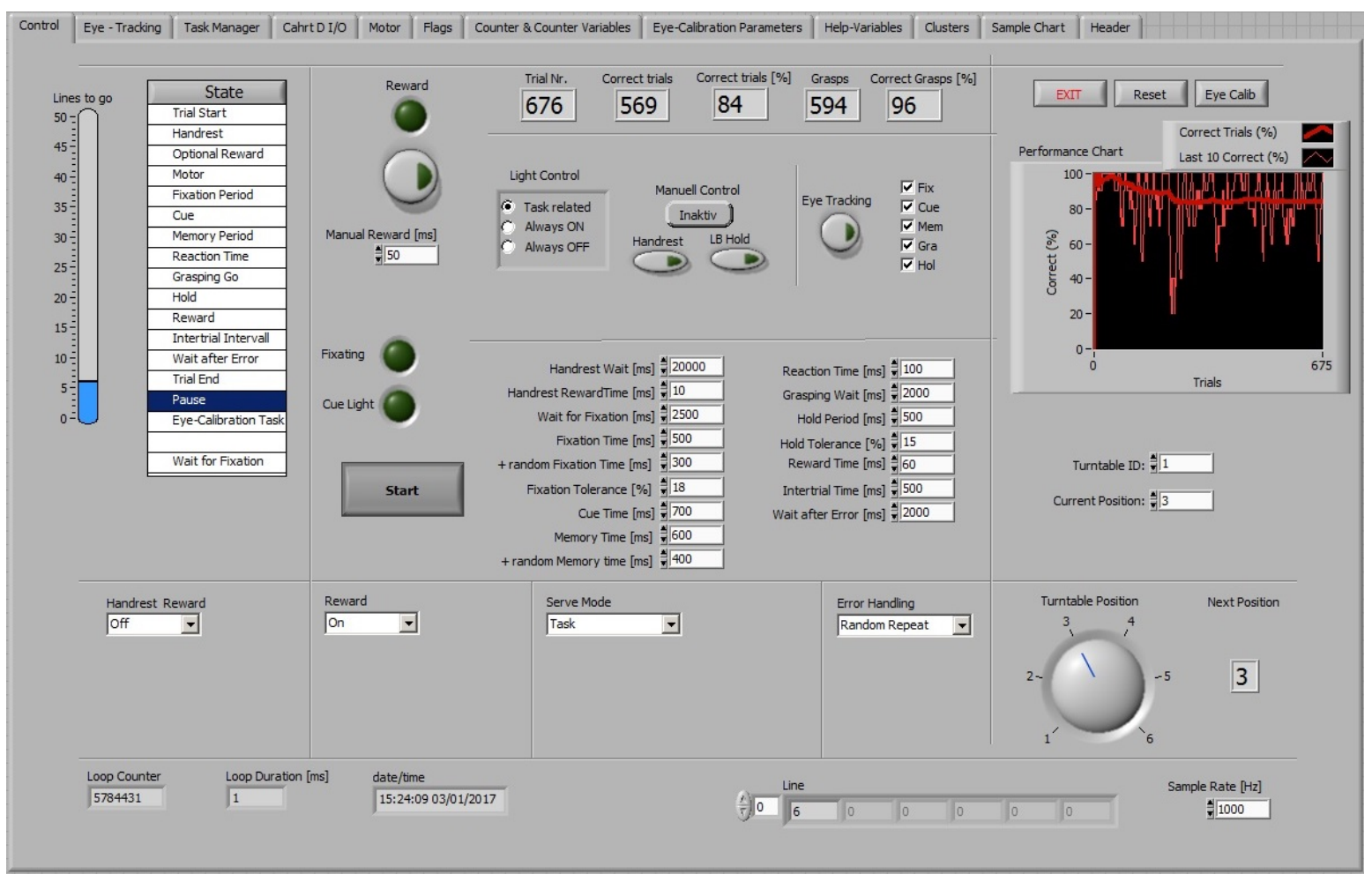

Figure 2.4: LabVIEW VI-Decoding Task - The main page of the LabVIEW VI that controls and monitors the Motor decoding task in real-time (1ms iteration time). It allows to set the timings of all the trial epochs, the order in which objects are presented, which LED's turn on, etc. Also the performance can be monitored. Last but not least it transmits all it's vital parameters to the Cerebus system to be synchronised and recorded. 


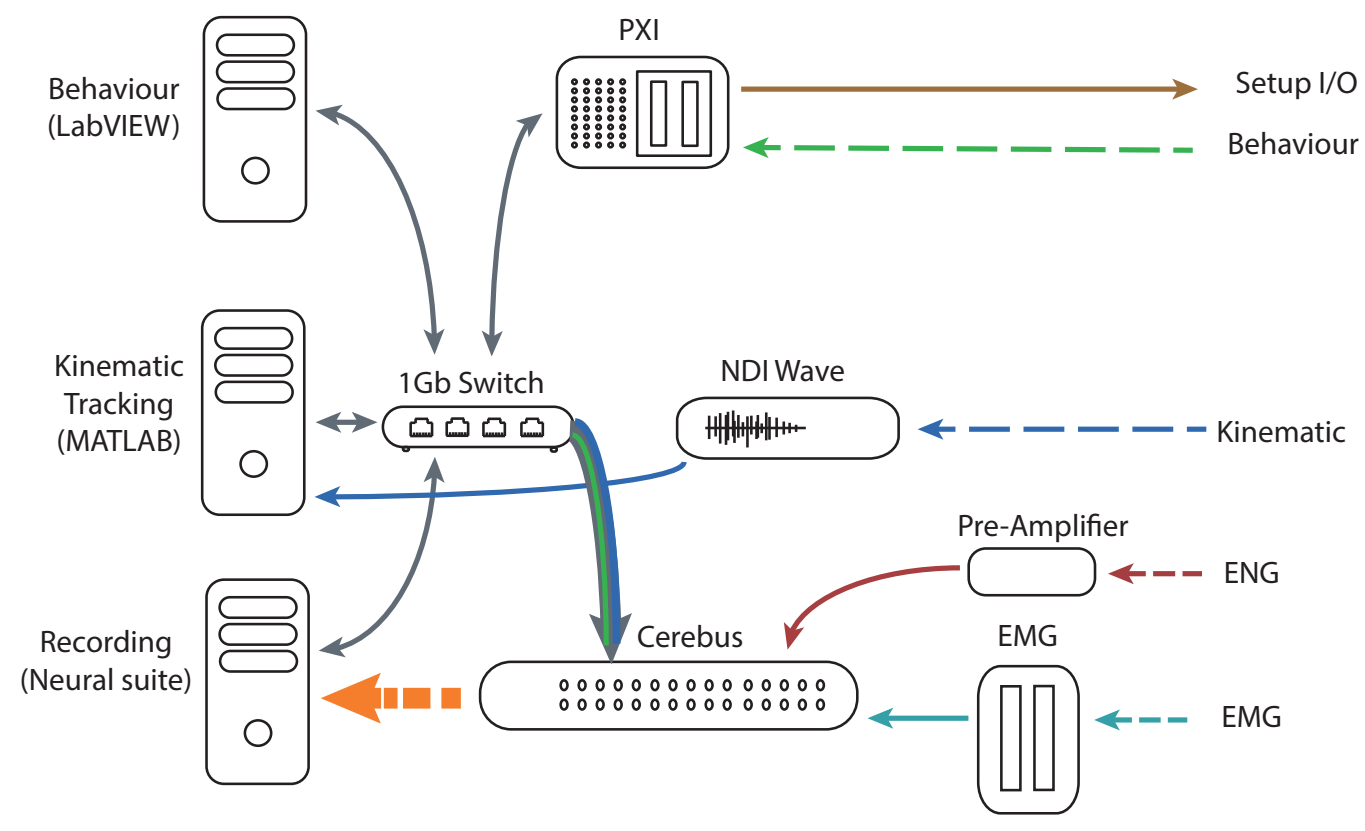

Figure 2.5: Setup Overview - Motor task - Three individual PC's control the behavioural settings and monitoring of the setup, the kinematic tracking and the recording of the data. All data is synchronised by the Cerebus system and then saved on the Recording PC. 


\section{Hand kinematics}

As mentioned before the hand kinematics of the animal are tracked in 27 DOF. The hand tracking is done on a separate PC, running the KinemaTracks software Schaffelhofer and Scherberger, 2012] based on the Wave tracking system (Northern Digital Inc.). The advantage of using this electromagnetic field tracking system is that it allows kinematic tracking, without camera or light dependency. This enables continuous tracking even when the hand is obscured by the table or object.

For hand tracking the monkey was trained to wear a fingerless glove with 6 electromagnetic sensor coils, mounted on the finger tips and wrist to track 3D position and orientation of the finger tips and a reference sensor on the back of the hand (see Figure 2.6). A hand model in the software used the hand parameters (digit lengths and sensor coil location relative to the finger tips, etc) in combination with the physical and anatomical constrains of the primate hand to reconstruct the angles of the $27 \mathrm{DOF}$ of the hand and arm joints with a temporal resolution of $100 \mathrm{~Hz}$.

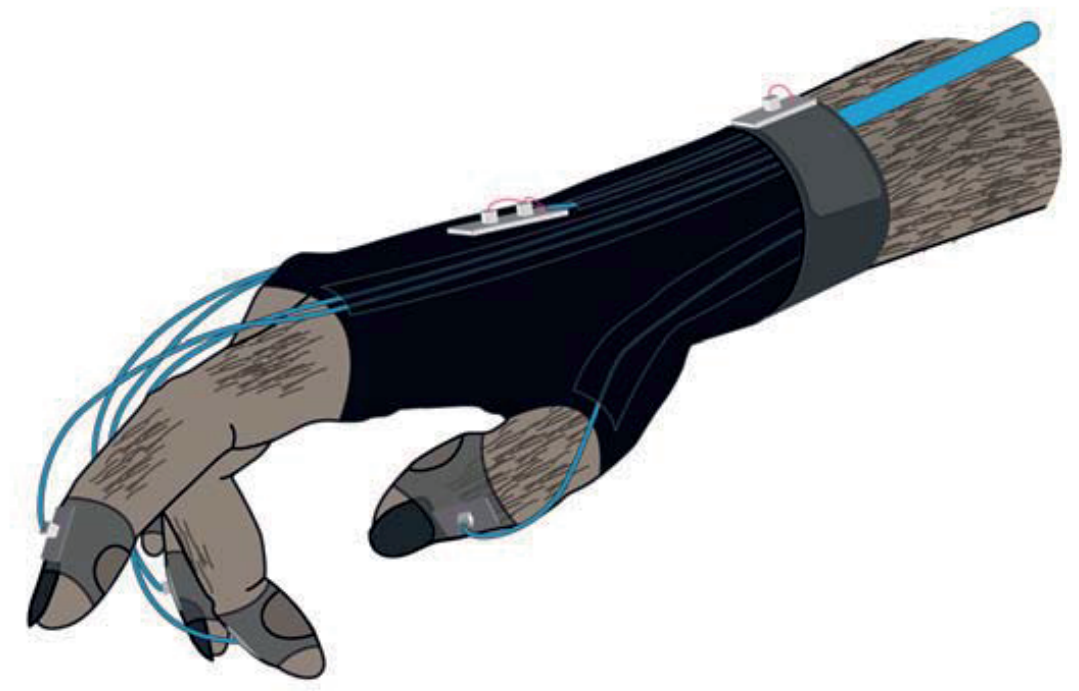

Figure 2.6: Hand Tracking Glove - The tracking system contains of a electromagnetic field generator and a fingerless glove (see image) which holds a reference sensor on the back of the hand, 5 flexible fingertip rings each their respective sensor and a wrist band with another sensor. Figure taken from [Schaffelhofer and Scherberger, 2012]. 
The following joint angles can be reconstructed: flexion/extension and abduction/adduction of all 5 digits; flexion/extension, pitch, roll and yaw of the wrist; flexion/extension of the elbow; pitch, roll and yaw of the shoulder (for details, see Table 2.2).

Table 2.2: Tracked Degrees of Freedom - Hand tracking system

\begin{tabular}{|l|l|l|}
\hline Joint & DOF & Movement \\
\hline Thumb & 4 & $\begin{array}{l}\text { - flexion/extension: } \\
\text { carpometacarpal (CMC) } \\
\text { metacarpophalangeal (MCP) } \\
\text { interphalangeal (IP) } \\
\text { - abduction/adduction }\end{array}$ \\
\hline $\begin{array}{l}\text { Index } \\
\text { Middle } \\
\text { Ring } \\
\text { Little }\end{array}$ & 16 & $\begin{array}{l}\text { - flexion/extension: } \\
\text { carpometacarpal (CMC) } \\
\text { metacarpophalangeal (MCP) } \\
\text { interphalangeal (IP) } \\
\text { - abduction/adduction }\end{array}$ \\
\hline Wrist & 3 & $\begin{array}{l}\text { - pitch (flexion/extension) } \\
\text { - roll (pronation/supination) } \\
\text { - yaw (abduction/aduction) }\end{array}$ \\
\hline Elbow & 1 & - flexion/extension \\
\hline Shoulder & 3 & $\begin{array}{l}\text { - pitch (flexion/extension) } \\
\text { - roll (lateral/median rotation) } \\
\text { - yaw (abduction/aduction) }\end{array}$ \\
\hline Total: & 27 & \\
\hline
\end{tabular}

\subsubsection{Behavioural paradigm}

The animal initiates a trial by pressing the handrest button with it's active grasping hand. A red LED then shines, during which the turntable rotates to a single (preset) object in a pseudo-random fashion. After a random fixation period $500 \pm 400 \mathrm{~ms}$ the object is shortly lit with a white LED array. The latter step is introduced for the application in cortical recordings, during which controlling the presented visual information is crucial. As the visual system does not affect the neural activity in the PNS of the arm, this step is not as important for the current experiments. I still chose to train the animal in the dark as it enhances the focus of the animal to the behavioural cues, i.e. the red cue LED and the objects. The red LED will then blink, 
which indicates the start of the go-phase, in which the animal has $1000 \mathrm{~ms}$ to grasp and lift the object. If the object is lifted correctly for $500 \mathrm{~ms}$, an auditory sound is presented marking the successful trial and the animal receives a liquid reward through a tube.

\subsubsection{Neural and EMG recordings}

The neural recordings were performed with a 128-channel recording system (Cerebus, Blackrock Microsystems) of which two banks (each 32 channels) were sufficient to cover the two (12channel) arrays. The metal headpost of the monkey served as common electrical ground. Neural data was recorded at $30 \mathrm{kHz}$ with a 0.3-7500 Hz hardware filter. Additionally a $100 \mathrm{~Hz}$ high pass filter was used to prevent amplifier clipping. As mentioned in section 2.1, the TIME arrays use a 16-pin Omnetics connector. With a custom-made adapter they fit to the 36-pin Omnetics connector of the Blackrock preamplifiers.

The two channel EMG recording was acquired with the NL844 preamplifier and NL820 Isolator (Neurolog Systems, Digitimer) and recorded at $2 \mathrm{kHz}$ with the Cerebus system. In MATLAB it was subsequently band-pass filtered (25-250 Hz, 3th order Butterworth), rectified, smoothed (Gaussian, $\sigma=10 \mathrm{~ms}$, binsize $=2.5 \sigma$ ), and normalised to the activity during the Fixation epoch Farrell and Weir, 2005.

\subsubsection{Neural signal analysis}

\section{Pre-processing and denoising}

Raw neural signals are passed sequentially through a non-causal low-pass median filter and a non-causal low-pass Butterworth filter $(5 \mathrm{kHz})$ to denoise the signal. To further remove recordings artefacts, principal component analysis (PCA) was applied on all recording channels. Reconstruction of the neural recordings was achieved by removing the main PCA components that are present commonly in all channels, which preserves the unique information of each channel. 
PCA is normally used for dimensional reduction: representing a majority of the data using a fraction of the variables. When recording from an array in either the brain or nerve tissue, it is unlikely that the a biological signal would appear on all channels at exactly the same time. Noise, from movement or another external source, however, can appear simultaneously on all channels, which would result in a common PCA component after the PCA analysis. Substracting this component from all the channels before reverse PCA-ing the data can be used to clean up the signal before spike sorting. To prevent that entire channels would be filtered out, all principle components (PCs) with a coefficient greater than 0.36 are kept. An overview of the data pre-processing is shown in Figure 2.7.

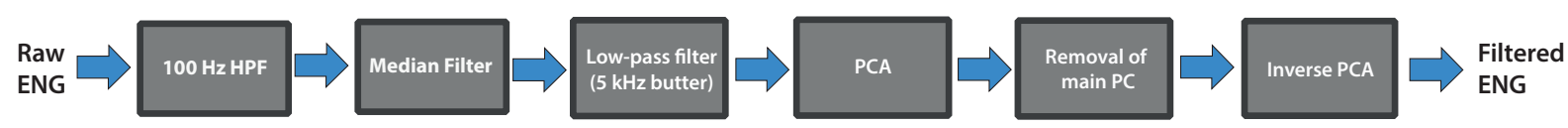

Figure 2.7: Data Pre-Processing - Block diagram of the pre-processing phase. Raw neural signals are first run through a median and low-pass Butterworth $(5 \mathrm{kHz})$ filter. PCA of the data in all channels allows the identification of the main common components between the channels. After removal of these PCA components the inverse PCA process leaves you solely with unique information in each channel.

\section{Spike detection and sorting}

For the offline spike sorting process a modified version of the MATLAB toolkit 'WaveClus' was used Quiroga et al., 2006, Dann et al., 2016. It is a fast and unsupervised spike sorting algorithm, based on sorting with wavelets and superparamagnetic clustering. It provided an unsupervised solution that can be further optimised manually. This allowed the detection of single units in a multi-unit channel and also allowed the removal of artefacts that are falsely identified as spikes. An overview of the spike detection and sorting is shown in Figure 2.8.

Multilevel amplitude thresholding is used to detect the spikes in the neural recordings. The following thresholds $\left(T h r_{x}\right)$ are applied:

$$
\begin{gathered}
\sigma=\operatorname{median}\left(\frac{|x|}{0.6745}\right) \\
T h r_{\text {lower }}=5 \sigma \\
T h r_{\text {upper }}=100 \sigma
\end{gathered}
$$




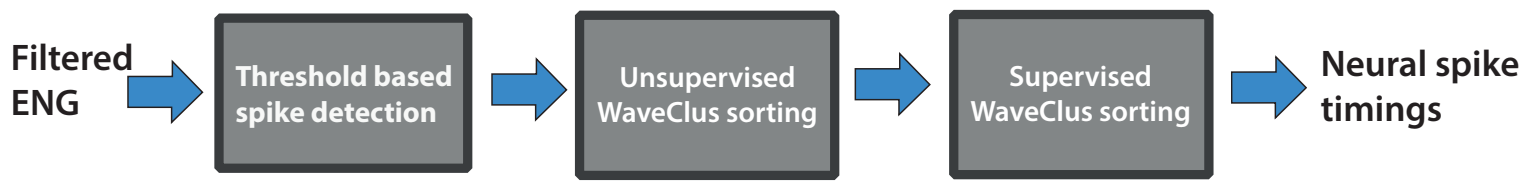

Figure 2.8: Spike Sorting - Block diagram of the spike detection and sorting phase. Spikes are detected in the filtered neural signals by applying a multilevel threshold. Unsupervised sorting by WaveClus provides an initial sorting solution, followed by a supervised WaveClus session, which optimises the neural spike timings output.

Where $x$ is the filtered neural signal and $\sigma$ the estimation of the standard deviation of the background noise. The estimate of the standard deviation is chosen over the actual standard deviation, because it is less sensitive to high firing rates and large spike amplitudes.

The spike sorter then localises distinctive spike features, using a combination of wavelet transform, PCA and the original waveforms. Based on these features, a normality estimation (Lilifors test) selects the features. Superparamagnetic clustering then gives so called 'temperatures', which are different cluster configurations. These temperatures are a measure for the ability to either separate narrow waveforms (low temperature) or wide ones (high temperature). Browsing the features through these temperatures, one can select a number of templates that best match the waveforms present in the recording channel. The unassigned waveforms are then matched with these templates using LDA (linear discriminant analysis) Dann et al., 2016].

\subsubsection{Object Classification}

To investigate if the neural recordings from both the median and ulnar nerve contain enough information to predict which object is grasped by the animal, I looked at the spike timings, during the movement as well as the hold phase. Summing and averaging all the spike events during each of these epochs gives us the average fire rate during this specific period of time. This is fed to a Linear Discriminant Analysis (LDA) classifier. Note that only the neural events during successful trials are used for decoding. This process is cross-validated 10 -fold, i.e. the data is split randomly into ten data sets of equal length and each section is then used once as testing data, while with the other 9 the classifier is trained. 


\subsection{Somatosensory Discrimination Task}

\subsubsection{Basic procedures}

One purpose bred female rhesus macaque $(7,8 \mathrm{~kg})$ was trained to perform a two-alternative forced choice discrimination task. It received stimulation to the median and ulnar side of the hand. By means of a button press the animal indicated which side of the hand was stimulated the strongest, in order to receive a liquid reward.

During the training phase tactile vibration stimuli were applied to the index and little finger with a custom developed vibration glove that the monkey wore. After the training phase, the animal was implanted with a TIME electrode in both the median and ulnar nerve of the upper arm. The tactile vibration stimuli were then substituted by electrical stimulation to the corresponding nerve. By varying the stimulation intensities between the median and ulnar nerve, by changing the pulse amplitude or the stimulation frequency, the sensitivity to the stimulation was then investigated.

\subsubsection{Experimental setup}

When stimulating the nerve to evoke sensory percepts, the stimulation parameters like, pulse width, amplitude and frequency all affect how the stimulation is perceived by the subject. With this experiment, I investigated how electrical stimulation to the PNS is perceived in terms of sensitivity and how well I can manipulate this sensation by tweaking the stimulation parameters. A big challenge in working with (non-human) primates, in comparison to humans, in somatosensory tasks, is that one cannot simply ask an animal how electrical sensory stimulation is perceived. This is also not a question that is addressed directly in this experimental task, but instead I try to answer how the sensitivity is modulated by the stimulation parameters.

It is known that similar patterns can be distinguished if delivered to a single channel / region of the hand. As [Romo and Salinas, 2001] indicated, sequential stimulation gives rise to simple binary higher/lower classification of the second stimulation compared to the first, without taking 
the 'baseline' into account. With simultaneous stimulation you eliminate this problem as both cues need to be taken into account. Such comparison of electrical stimulation to two different nerves has not been investigated yet. The task presented here, does not only allows us to train a macaque for differentiating graduated somatosensory cues, but also spatially differentiated ones (median and ulnar hand region). Simultaneous stimulation also comes closest to natural percepts, as it's rare that only a single finger is receiving sensory input.

The median and ulnar region of the hand were chosen as stimulation targets as the palmar side of the hand is more important for somatosensory exploration than the back side (which is innervated by the radial nerve). For training, the index and little fingers were chosen as targets for the tactile vibration stimulation. As described before, the median nerve innervates the thumb, index, middle and half of the ring finger. The ulnar nerve in turn innervates the other half of the ring finger and the little finger. To maximise the distinction between the two zones, we preferred stimulation sites (i.e. fingers) opposed from each other as far as possible. These would ideally be the thumb and little finger, but the short and stubby macaque thumb is not suitable for mounting the vibration motors on, leaving the index finger as the best alternative.

As mentioned before, I chose vibration tactile stimulation as the somatosensory modality over, for example, pressure, temperature or another somatosensory percept. This choice was made based on a few criteria. First, the sensory percept should be easy to apply, but also be safe to the animal. With sensory modalities like pressure and temperature you could inflict pain if there is a hardware defect and the stimuli is too strong. With small amplitude vibrations you do not have that risk. The second criteria is that it should have a high temporal resolution: there should be minimal delay between onset of the stimuli and the stimuli reaching the desired intensity. Finally, a solution that could be mounted on the monkey's hand was preferred, as this would allow the animal to move its arm around freely, thus reducing potential stress for the animal. Vibration as sensory modality fulfilled all these requirements and was therefore chosen. 


\section{Vibration glove}

To allow the training of the somatosensation discrimination task prior to the implantation of the PNS arrays, I designed a vibration glove that the monkey wears during the task (see Figure 2.9. This glove is similar to the hand tracking glove described in the Motor Decoding Task (see Section 2.3.2), with the difference that on the fingers there are now vibration motors (Swissphone Quattro Hurricane) mounted instead of the Wave System sensors.

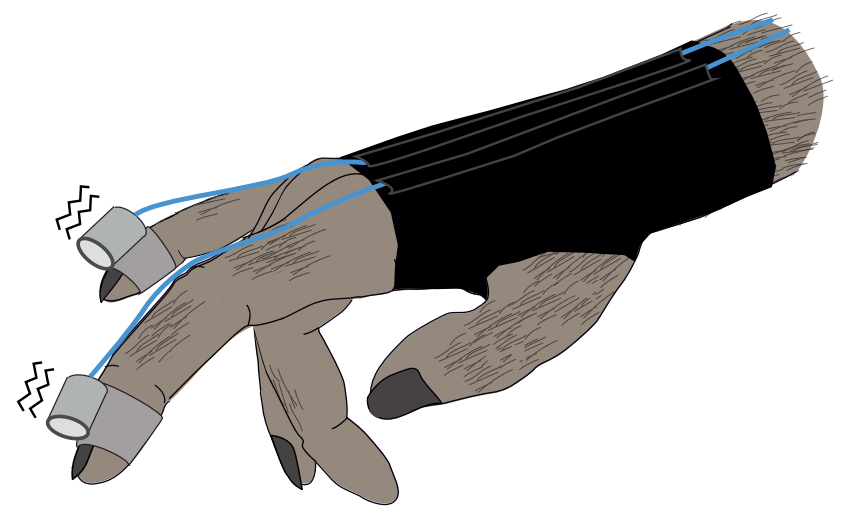

Figure 2.9: Vibration Glove - The rhesus macaque wears a custom made glove that routes and holds two vibration motors mounted on silicon rings. These rings are slid on the index and little finger of the hand and can provide tactile stimulation to these specified regions of the hand

The choice for these motors (see Figure 2.10 and Table 2.3 for specifications) was based on the size, ease of use and price. The first is important as the macaque hand is much smaller than the human hand. The latter two (ease of use and price) are influenced by the frequency of repairs required during the monkey training phase. In the end picking suitable vibration motors for the vibration glove, was a compromise between size and accuracy. On the one hand, the motors need to be small enough to be able to be mounted on the last phalanx of a rhesus macaque monkey's finger without obstructing the movement of the animal. But as you go smaller in size with the motors, the manufacturing tolerances of the motors play a larger role and a larger variety in turning speed between motors was found. Ensuring a consistent stimulation intensity during the trials is important for the experimental task design. After considerable testing (see Supplement: A.1) it was found that when two motors with similar voltage-frequency curves are found, then we can say with $95 \%$ confidence that with $0.5 \mathrm{~V}$ step sizes difference (LabVIEW 
Table 2.3: Technical specifications - Vibration motor (Swissphone Quattro Hurricane)

$\begin{array}{ll}\text { Width x height } & 4,5 \times 4,5 \mathrm{~mm} \\ \text { Radius } & 4,0 \mathrm{~mm} \\ \text { Body length } & 8,5 \mathrm{~mm} \\ \text { Total length } & 11,6 \mathrm{~mm} \\ \text { Vibration head radius } & 3,8 \mathrm{~mm} \\ \text { Vibration head width } & 2,0 \mathrm{~mm} \\ \text { Weight } & 0,5-1 \mathrm{~g} \\ \text { Impedance } & 45 \mathrm{Ohm} \\ \text { Operating Range } & 0,7-3.0 \mathrm{~V} \\ & 10-65 \mathrm{~mA}\end{array}$

output voltage) the frequency difference is $\sim 15 \mathrm{~Hz}$.

The motors are voltage controlled by the LabVIEW PXI box (see Figure 2.11). The glove can easily be upgraded to fit more vibration motors (e.g. one for each finger), if a more complex task design is desired. For this task two motors were sufficient.

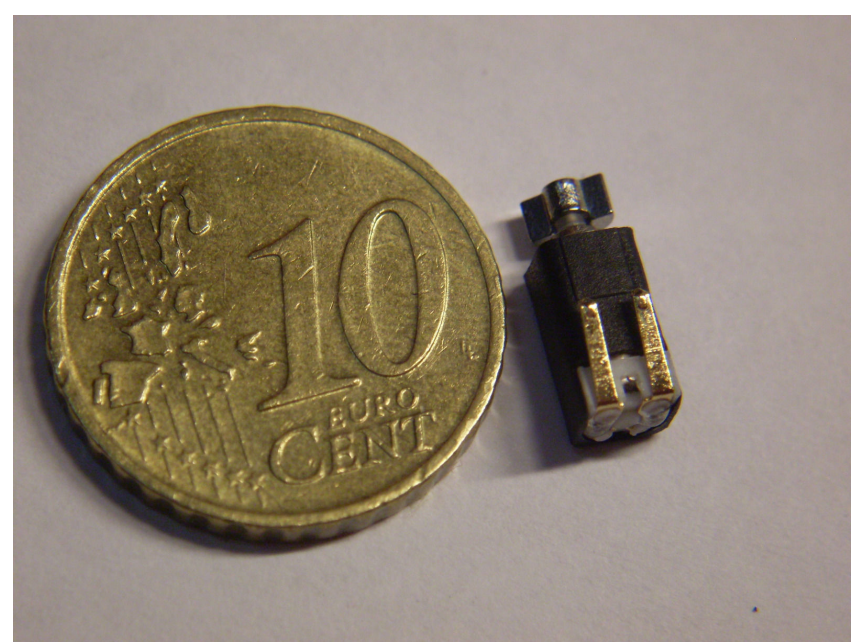

Figure 2.10: Vibration Motor - Close-up of the vibration motor (Swissphone Quattro Hurricane) used for the vibration glove

Stimulation and Behavioural control The somatosensory discrimination task was controlled through a LabVIEW VI. Apart from vibration motor control, this VI also controls the rest of the behavioural paradigm, including triggers and rewards, as well monitors the performance. This data is all synchronised with the behavioural parameters of the recording system. An overview of the VI main page is visible in Figure 2.12 and a data flow diagram with all components in the setup is shown in Figure 2.13. 


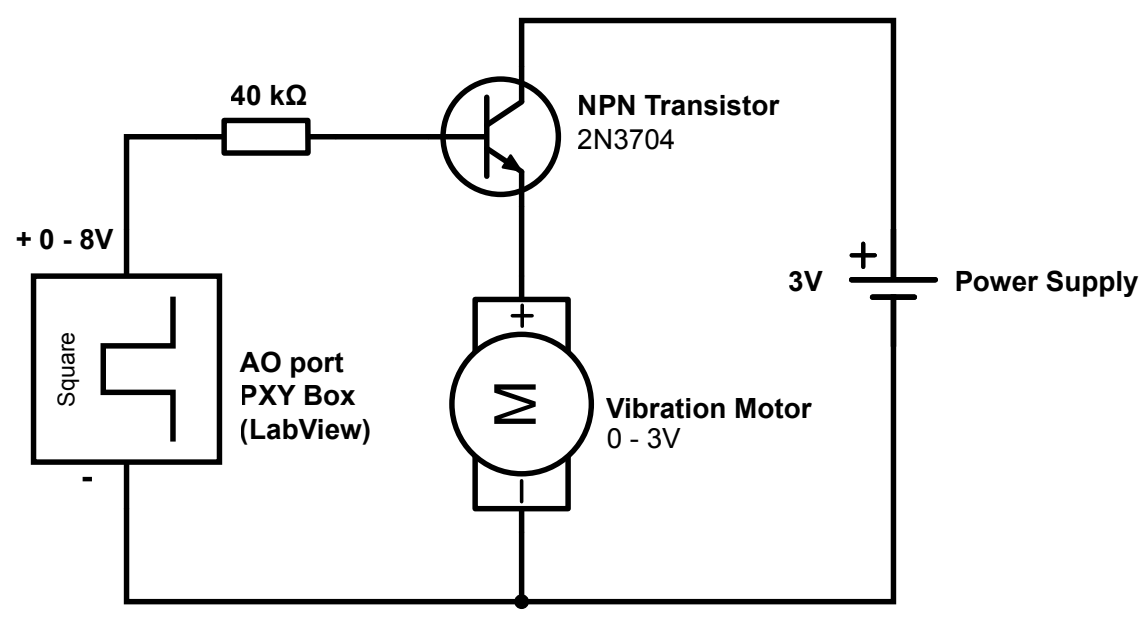

Figure 2.11: Wiring Schematic Vibration Motors - The motor is driven by a $3 \mathrm{~V}$ power supply, that is gated by a NPN Transistor (2N3704), driven by an analog output port of the LabVIEW PXI Box. The 0-8V DC voltage determines how much voltage is supplied to the Vibration motor, thus controlling its speed, thus controlling the stimulation intensity.

As mentioned before, the behaviour PC sets the stimulation parameters through the PXI box, however, as can be seen in Figure 2.13, a separate PC (Stimulation) is controlling the CereStim96 stimulator. The reason for this (seemingly) elaborate setup is that the CereStim96 cannot be controlled through LabVIEW. The stimulator can be triggered through one the PXI box I/O ports, however, this does not allow its parameters to be changed: a crucial part of the somatosensory discrimination task. The software package that came with CereStim96 is sufficient for single parameter stimulation but also is not fit for on the fly parameter switches, nor is it fit for stimulating two (or more) channels simultaneously. The latter could be initiated by the MATLAB application programming interface (API). I therefore decided to write a custom MATLAB GUI for this purpose (see Figure 2.14), which does allow communication with the PXI box. The LabVIEW VI for the somatosensory task (see Figure 2.12), sends the relevant stimulation parameters (channel number, pulse number, amplitude, phase width, interphase time, frequency and activation state) over an User Datagram Protocol (UDP) stream to the stimulation PC. The MATLAB CereStim GUI then interprets the UDP stream and sets the stimulator parameters accordingly. The GUI is also used to read out the electrode contact impedances, using the build-in impedance measurement function of the CereStim96. 


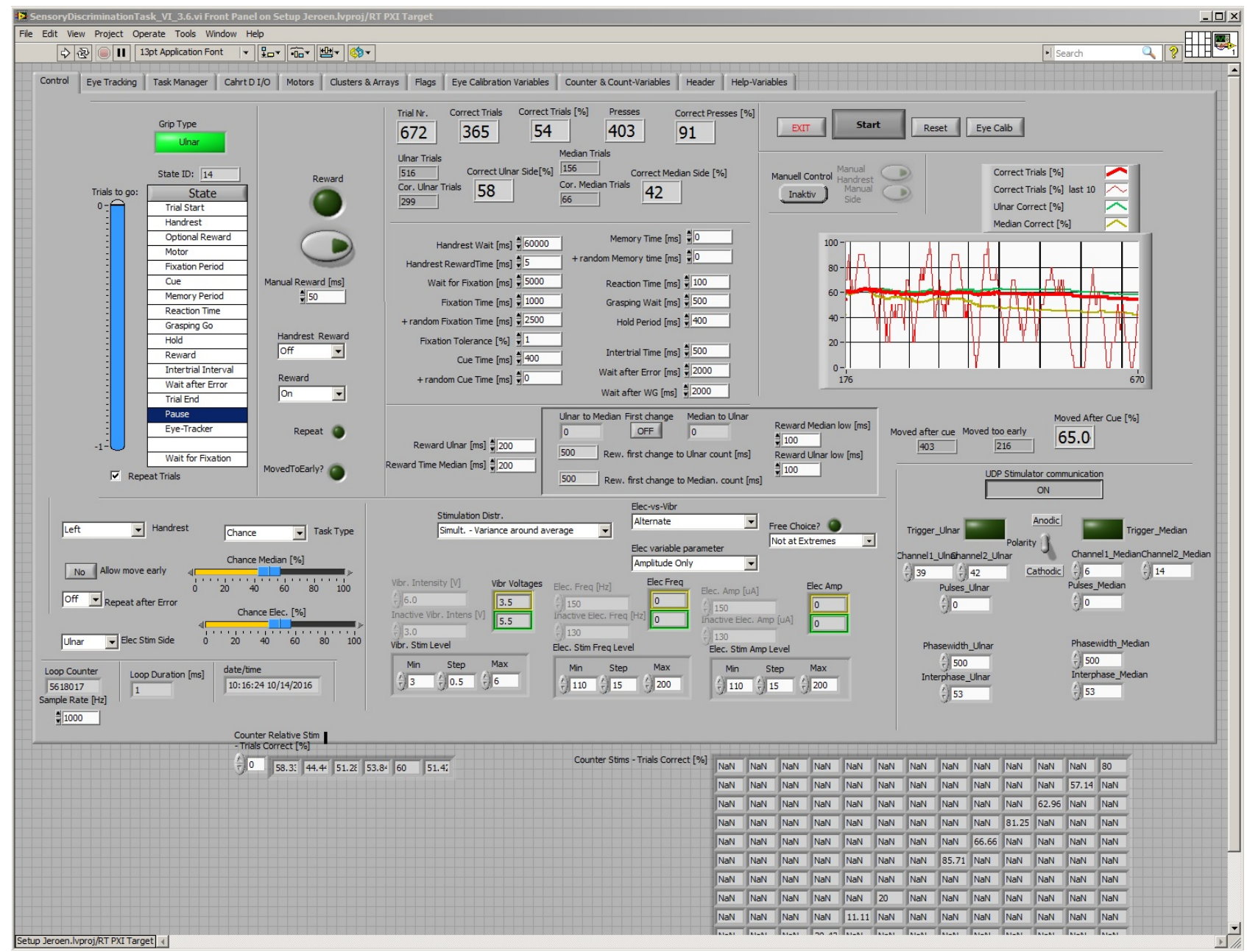

Figure 2.12: LabVIEW VI-Sensory Task - The main page of the LabVIEW VI that controls and monitors the somatosensory discrimination task in real-time (1ms iteration time). It allows to set the timings of all the trial epochs, the order in which objects are presented, which LED's turn on, etc. Also the performance can be monitored. It also transmits all its vital parameters to the Cerebus system to be synchronised and recorded. 


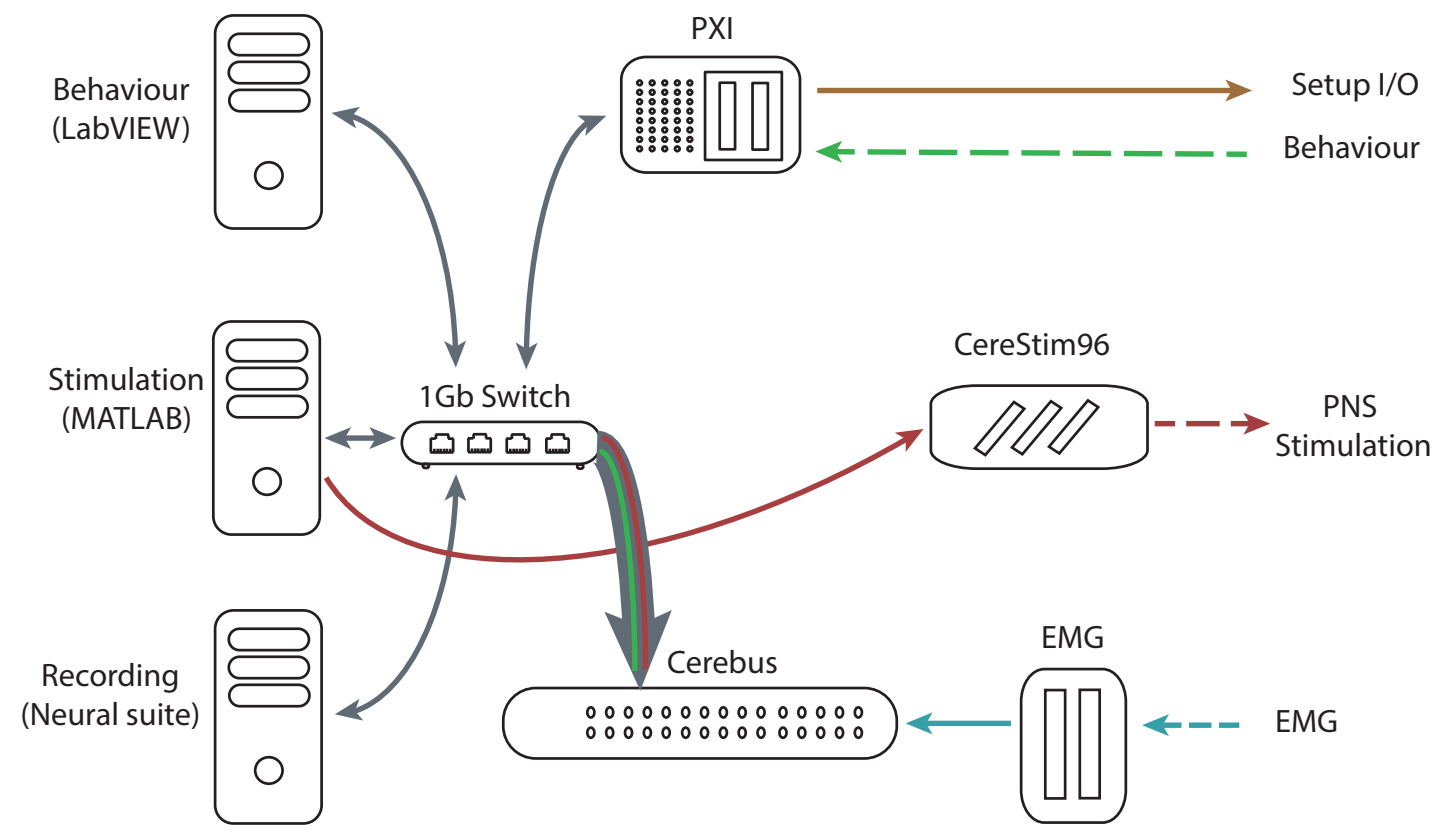

Figure 2.13: Setup Overview - Sensory - Three individual PC's control respectively the behavioural settings and monitoring of the setup, the kinematic tracking and the recording of the data. All data is synchronised by the Cerebus system and then saved on the Recording PC.

\subsubsection{Behavioural paradigm}

\section{Initial training paradigm}

Initially we had a different choice paradigm in mind to allow the monkey to indicate it's choice. The animal in question was already trained for a delayed grasping task on a grasping box: a handle that can detect whether a power grip (full hand) or precision grip (index-thumb opposition) is performed on it (see Figure 2.15). As this is also a binary choice instructed, but instructed with a LED cue instead of a tactile cue, we hoped to shorten the training time by adapting this task into one suitable for somatosensory discrimination. The idea was to slowly introduce the vibration during the LED cue phase, so the monkey learns to accustom the new sensation and learns to associate median (index finger) stimulation with the yellow LED and ulnar (little finger) stimulation with the green LED. Then by gradually decreasing the LED cue time, we could shift the choice indication to pure vibration. However, it soon became clear that the animal did not make the association between the two types of cues. Up to a LED cue time 


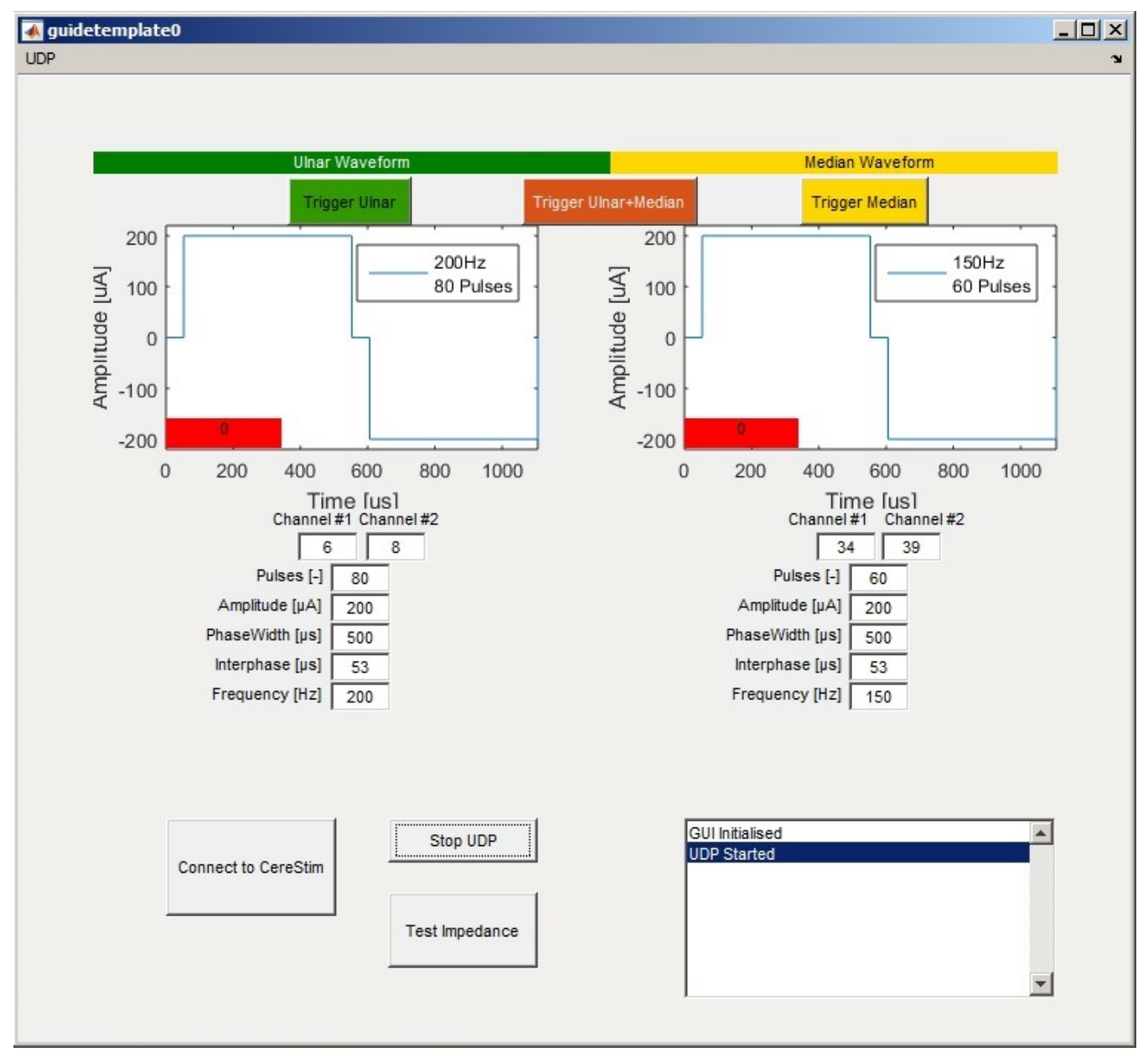

Figure 2.14: CereStim GUI - A custom MATLAB GUI written to control the CereStim96 stimulator and thus the electrical stimulation pulses applied to the median and ulnar nerve through the TIME arrays. Once connected to the CereStim96, it can receive UDP commands from the LabVIEW Sensory Task VI (Figure 2.12 and will set the stimulation parameters accordingly and applies a pulse (train) when triggered. Note that setting the parameters manually and/or manually triggering pulses (either an individual nerve or both simultaneously) is possible. This GUI is also used for the impedance measurements of the TIME electrodes.

of $20 \mathrm{~ms}$ the animal was able to perform the task with $400 \mathrm{~ms}$ vibration cue, but decreasing the LED even further, it became impossible to see and it became obvious that it was only watching the LED cue and ignoring the vibration stimuli. The failure to associate the two cues is most likely due to over-training with the original grasping box task: the animal could not dissociate the grasping box from the indication LED's.

A new temporary training paradigm was therefore introduced to make the animal aware of the vibration cues delivered to its fingers. This task only involved a handrest button and the vibration glove. Once of the vibration motors would spin, it had to react by lifting its hand from the button in order to receive reward. This task soon showed the rhesus macaque hands 


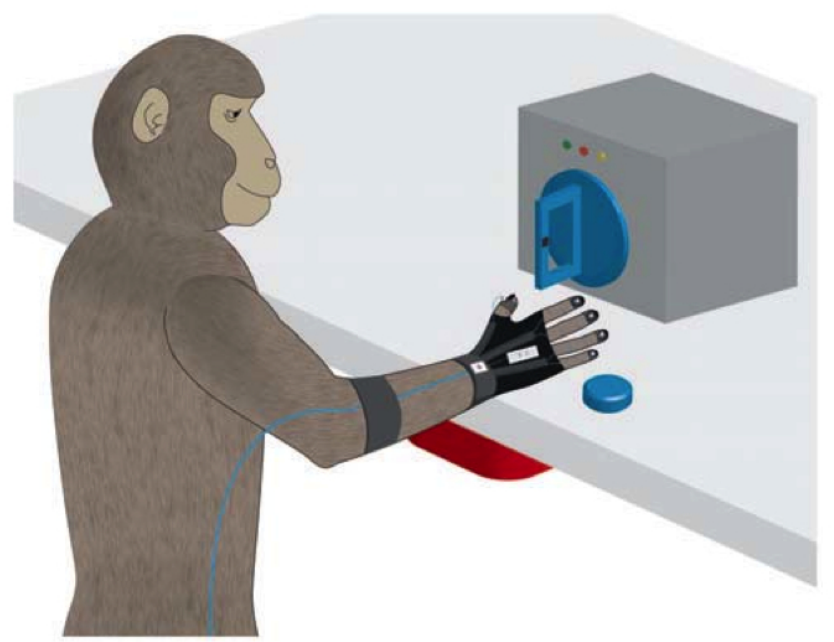

Figure 2.15: Graspbox Sensory Task - Initial idea for the behavioural choice indication in the sensory discrimination task was to let the monkey indicate whether the median or the ulnar side of the hand was stimulated more strongly, by performing respectively a power grasp or a precision grasp on the handle.

are very sensitive to vibration stimulation as it could already detect the vibration when the motor was hardly spinning (frequency $100 \mathrm{~Hz}$ ). This intermittent training task both allowed for exploring a comfortable (vibration) stimulation range for the animal as did it serve as a stepping stone to the final training paradigm.

\section{Final training paradigm}

Now that it was clear that the monkey learned to pay attention to the vibration cues applied to his fingers. It was decided to design a complete new task that was completely disassociated with any previous experiments the monkey was trained for. Making an eye saccade to the left or right was considered a possible choice indication, since the monkey had experience with eye fixation and it was a quite unbiased and non-strenuous action for the animal. However, to train this, it would require the monkey to be head fixated; something we had planned only do shortly before the implantation to avoid possible complications (e.g. infections of the wound margin) that are involved with having a head cap. Not even considering the actual training time for the eye fixation, this would already put another delay on the project as the training of the animal could not move forward until the animal was given a head cap.

I therefore decided to move to a button press choice task. To indicate whether the median 
or ulnar stimulation was more intense, the monkey had to press a button on the median and ulnar side of the hand rest button, respectively. This was first trained with a strong vibration stimulation to the ulnar side of the hand, and the animal had to a button now instead of just lifting the hand. Once learned, the other side was trained, followed by blocks of either stimulation. These blocks were incrementally made smaller till the monkey could perform the task with random selected stimulation side. From this point simultaneous stimulation was introduced, in which a stimulation with different intensities was applied simultaneously to both hand regions. Initially completely random stimulation were chosen, but as the motor accuracy (see Supplements A.1) could not be guaranteed to be highly consistent between motors, it was chosen to use increments of $\sim 15 \mathrm{~Hz}(0.5 \mathrm{~V}$ on the LabVIEW Analog Out port) to drive the motor. This increment step would ensure that one stimulation frequency of the motor would definitely be different between the two motors.

An overview of the final somatosensory discrimination task can be seen in figure 2.16. A trial is initiated by pressing the centre hand rest button. After a $1000 \mathrm{~ms}+$ random 0-2500 ms time delay, a $400 \mathrm{~ms}$ stimulation is given to the hand, either by the vibration motors attached to the hand, or through electrical stimulation of the median/ulnar nerve through the TIME electrodes implanted in these nerves. After the cue phase, the go phase is initiated in which the monkey has to indicate which of the two stimuli was perceived stronger by pressing the button on the respective side of the centre hand rest button. When chosen correctly, the monkey received a reward in the form of fruit juice.

In case of tactile vibration stimulation, there is a clear and objective criteria that marks the strongest stimulation: the higher the motor frequency the stronger the motor vibration intensity is. In case of the electric stimulation you might be inclined to say the stimulation with the biggest charge is per definition the strongest stimulation. This may be true in an electrical engineering point of view, but the intensity perception of this stimulation by the monkey might be influenced by a couple of other factors. Both nerves have a different size and fascicular organisation, it is also unknown where the active site is located in relation to the sensory fascicles. Finally, the amount of nerve fascicle activation is not just a product of the electrical stimulation (pulse amplitude and phase width and pulse frequency), also the diameter of the 


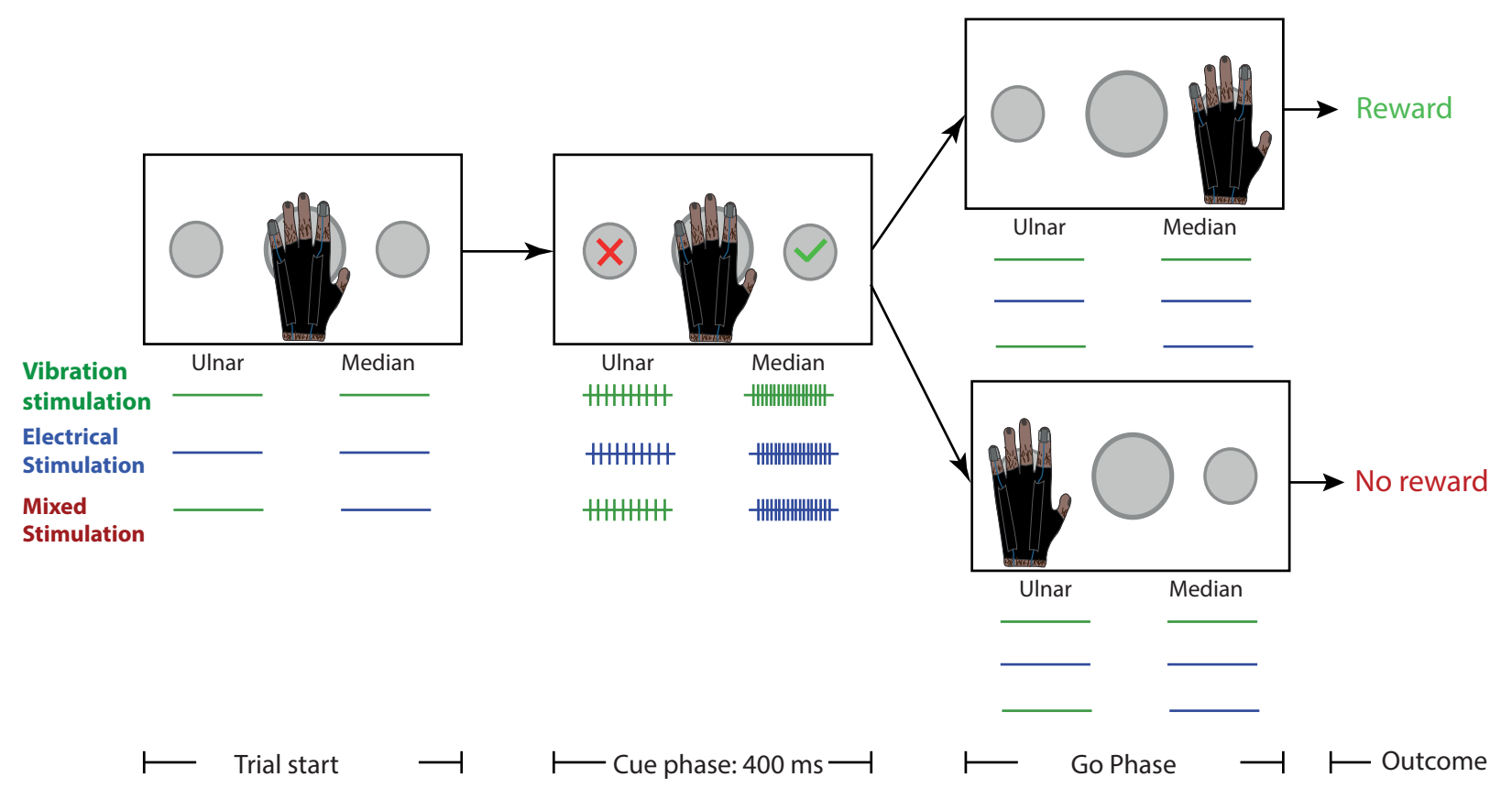

Figure 2.16: Somatosensory Discrimination Task - A trial is initiated by pressing the centre hand rest button. After a random length fixation period, either a tactile (vibration) or an electrical (nerve) stimulation is applied to the ulnar or median region of the hand. These regions are stimulated simultaneously, but with different intensities. After the cue phase, the monkey has $500 \mathrm{~ms}$ to press the side button to indicate which side was stimulated the strongest. A liquid reward (grape juice) is given every time it indicates the correct side.

fascicle itself plays a role. Larger fascicles have a lower resistance and therefore are more easily activated by electrical stimulation. To investigate which stimulus is perceived stronger by the monkey, an obvious solution is to give the animal a free choice. However, during the training process one has to make sure the monkey does react properly to the electrical stimulation and indicates the correct side. Therefore it was decided to give the monkey only free choice when the stimulation parameters were very close to each other. When the vibration was at its lowest or highest setting, the animal had to choose the electrical and the vibration cue, respectively, in order to receive a reward.

\subsubsection{Somatosensory channel mapping}

As discussed before, the nerves are bidirectional information channels, transmitting both efferent signals to the muscles and afferent information back to the brain. To evoke somatosensation 
with electrical stimulation, one would ideally like to evoke only activity in the afferent fascicles, while leaving the efferent nerves unaffected. As there is no non-invasive way of telling which fascicles lead to where and during the implantation there is currently no way to influence which electrode contact sites will be in/near specific fascicles in the nerve. One has to investigate post-implantation, if a contact site is connected to a motor or sensory fascicle. This will require mapping of the electrode array sites.

The initial idea was to stimulate with vibration different fingers and regions on the hand and fingers while simultaneously recording the nerve activity. While the monkey is in rest, there should not be any motor activity present in the nerve, so any neural activity modulation during the vibration stimulation can be attributed to activity in sensory fascicles. By covering different locations on the hand, you can then create a sensory map of the nerve.

However, due to the poor recording capabilities (see Results) it was not possible to distinguish sensory from motor fascicles. To make sure that the electrical stimulation delivered during the somatosensory discrimination task was actually felt by the animal, we only stimulated channels that evoked a visible movement response. This ensured that actual neural activity was evoked by the stimulation and that we were not stimulating a dead channel.

\subsubsection{Data analysis}

The behavioural choice of the animal is the metric we are interested in to evaluate the animal's ability to discriminate the stimulation, i.e. which button the monkey chose to press after receiving two stimuli with different intensities. Performance was quantified as the percentage of correct trials for a given stimulation combination. To generate a psychometric curve, I took the percentage of ulnar choices made for the given stimulation combination, with $100 \%$ indicating only the ulnar button is pressed, and at $0 \%$ only the median button is pressed.

Aside from the button choice, the cue-onset and movement-onset times (handrest button released) were used to derive the reaction times of the animal. The reaction time can be used to check if the animal is guessing the onset of the go-cue or if it is actually reacting to the 
stimulation. This is especially important when stimulating near the detection threshold.

Finally, an attempt has been made to detect sensory neural information. The period of interest here was the cue epoch, when the hand stimulation (with vibration stimuli) was given in this epoch. If the recorded neural signals modulate with the neural response, we can tell more about the neural coding of these stimuli. The same data pre-processing and spike sorting steps were applied as in the motor decoding task (see Section: 2.3.5). Recording during this task seemed hopeful as there is no movement during the cue epoch, which could increase the chance of detecting neural activity. Even if no spiking activity is perceived, we could still see if there is any neural information present in the signal by looking at the power of the signal in the 800-1500 Hz frequency band Logothetis et al., 2001. For this analysis we started with the filtered neural signal described in Section 2.3.4, which is 800-1500 Hz bandpassed (4th order Butterworth). Next, the instantaneous power was calculated by squaring the signal of an 1,5 second time period (0,5 seconds pre cue-phase till 0,5 seconds post go-phase), which are binned for every stimulation pair (median vs ulnar vibration stimulation). Though the extensive filtering does suppresses a large amount of the artefacts that are present in the signal, it is still necessary to apply a upper threshold ( $\sim 20 \%$ above $\max$ (baseline)) to avoid that artefacts cause an upwards shift of the baseline power. After smoothing every sample in all the bins with a normal probability density kernel (width: $6 \sigma, \mu=3 \sigma$, with $\sigma=30 \mu s$ ) the average power for each bin was plotted.

\subsubsection{Electrical stimulation}

The median and ulnar nerve were electrically stimulated with the Cerestim96 stimulator (Blackrock Microsystems), which connected through the 36-pin-to-16-pin Omnetics to the TIME electrode arrays.

Current controlled biphasic pulses (amplitude: 20-210 $\mu \mathrm{A}$, phase width: $500 \mu$ s, interphase: $53 \mu \mathrm{s})$ were applied with frequencies between 100-200 Hz and pulse durations of $400 \mu \mathrm{s}$. The frequency range was chosen as it matched that of the vibration stimulation, in the hope that would also evoke a similar perception. For the pulse shape, biphasic pulses were favoured over 
monophasic ones, as the latter do not negate the build-up of charge in the surrounding tissue, which can cause severe tissue damage. Amplitude and phase width were determined by trial and error. Ideally, literature suggest, to keep the phase width of the pulse as short as possible and instead raise the amplitude instead. However, with shorter phase width's than $500 \mu$ s we were not able to evoke responses in the awake animal and with these settings we were already hitting both the electrode max injection current limits (120 nC, see Section 2.1.1) as well as the max stimulation amplitude of the stimulator. 



\section{Chapter 3}

\section{Results}

\subsection{Implantation techniques}

\subsubsection{Acute electrical stimulation with cuff and TIME electrodes}

A terminal experiment on an adult male rhesus macaque allowed the surgical exploration of the median nerve (N. medianus) and the ulnar nerve (N. ulnaris). The animal was placed in balanced general anaesthesia (induction: ketamine+robinul then intubation, gas and IV analgesia). First the nerves in the forearm were explored. The median nerve could not be located here, but stimulation (100-150 $\mu \mathrm{A})$ with a blunt and a cuff electrode to a different nerve elicited movements of the middle, ring and little finger, indicating that the ulnar nerve had been found. Secondly the nerves in the upper arm were explored. The ulnar nerve in the upper arm was approached by dissection between the biceps and the triceps muscle from them medial side in direction of the humerus. Placement of a cuff electrode and stimulation caused strong ulnar hand abduction (threshold: 100-120 $\mu \mathrm{A}$ ). The medial nerve in the upper arm was approached by dissection just medial to the biceps muscle and proximal to the elbow. Placement of a cuff electrode and electrical stimulation with a low threshold (40-50 $\mu \mathrm{A})$ caused strong finger movements (mainly thumb to middle finger). Placement of the cuff electrode more proximal showed higher stimulation thresholds (75-100 $\mu \mathrm{A})$. A TIME electrode was also placed at the distal medial nerve on the upper arm. Stimulation showed mainly pronation 
at a threshold of 60-100 $\mu \mathrm{A}$. Lastly the possibility to wire the electrodes under the skin was explored. It is not be possible to have the connector near the implantation site in the awake animal as there is no tissue it can be securely embedded in, nor can it be guaranteed that the monkey would not meddle with it. For this reason, the wires were routed underneath the skin to the head cap, where the connector can be embedded securely into the head cap bone cement. The electrode cable was tunnelled from the distal upper arm to the posterior side of the arm, from there to the middle of the back and to the posterior side of the skull. A total cable length of $\sim 50 \mathrm{~cm}$ is required to route it completely.

\subsubsection{Acute stimulation and recording with TIME electrodes}

The second terminal experiment was performed with a second adult male rhesus macaque and allowed further surgical exploration of the median nerve and the ulnar nerve. The animal was placed in balanced general anaesthesia (induction: ketamine+robinul then intubation, gas and IV analgesia). The median nerve in lower arm between the elbow and wrist was prepared. Electrical stimulation with various amplitudes was applied through a cuff electrode around the nerve at the proximal and distal forearm (see Figure 3.1). Both locations elicited noticeable thumb movements. While the nerve was stimulated with the cuff electrodes, the neural activity was recorded with the TIME electrode, which was placed in the medial nerve at the proximal side (see Figure 3.2). Electrical stimulation of the TIME caused thumb movements as well but much lower thresholds are required.

The same procedure was repeated for the ulnar nerve of the right hand between the elbow and the wrist. Electrical stimulation of the ulnar nerve caused little finger movements and again neural spikes were recorded with the TIME.

Figure 3.3 shows a section of the recording made with a TIME electrode while the nerve was stimulated distally with the cuff electrode. The stimulation pulses, causing the muscle twitches, are clearly visible, but using the spike detection algorithm also random spiking was detected between the stimulation pulses. These single spike events are extracted and marked on the same figure and their waveforms are displayed as well. In the 2.5 minutes that were recorded 


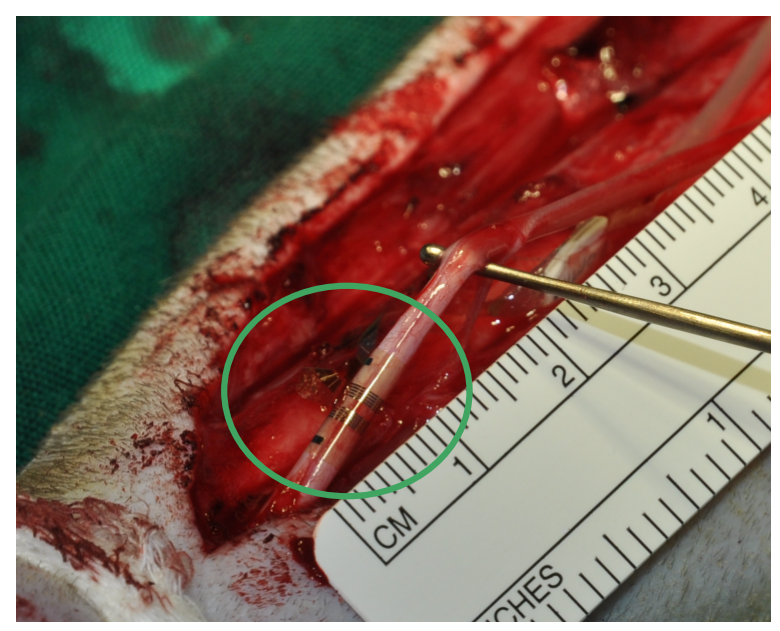

Figure 3.1: Cuff electrode implantation Surgical implantation of cuff electrode around the median nerve at the distal forearm.

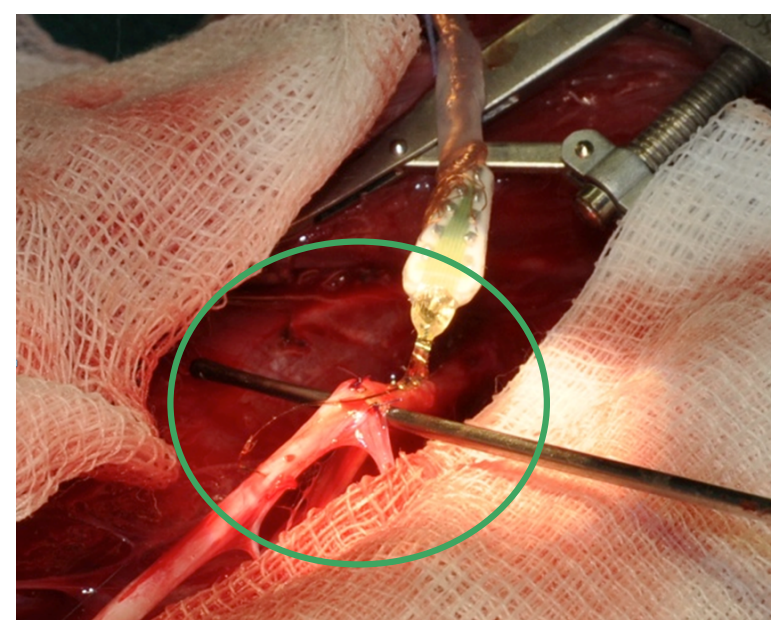

Figure 3.2: TIME array implantation Surgical implantation of TIME array placed in the median nerve at the proximal forearm.

with this specific channel and stimulation intensity, 517 single spikes could be detected.

From these acute (terminal) experiments we could conclude that the implantation technique of TIME electrodes in the PNS of rhesus macaques is feasible, including the placement of a subcutaneous cable and a cranial connector.

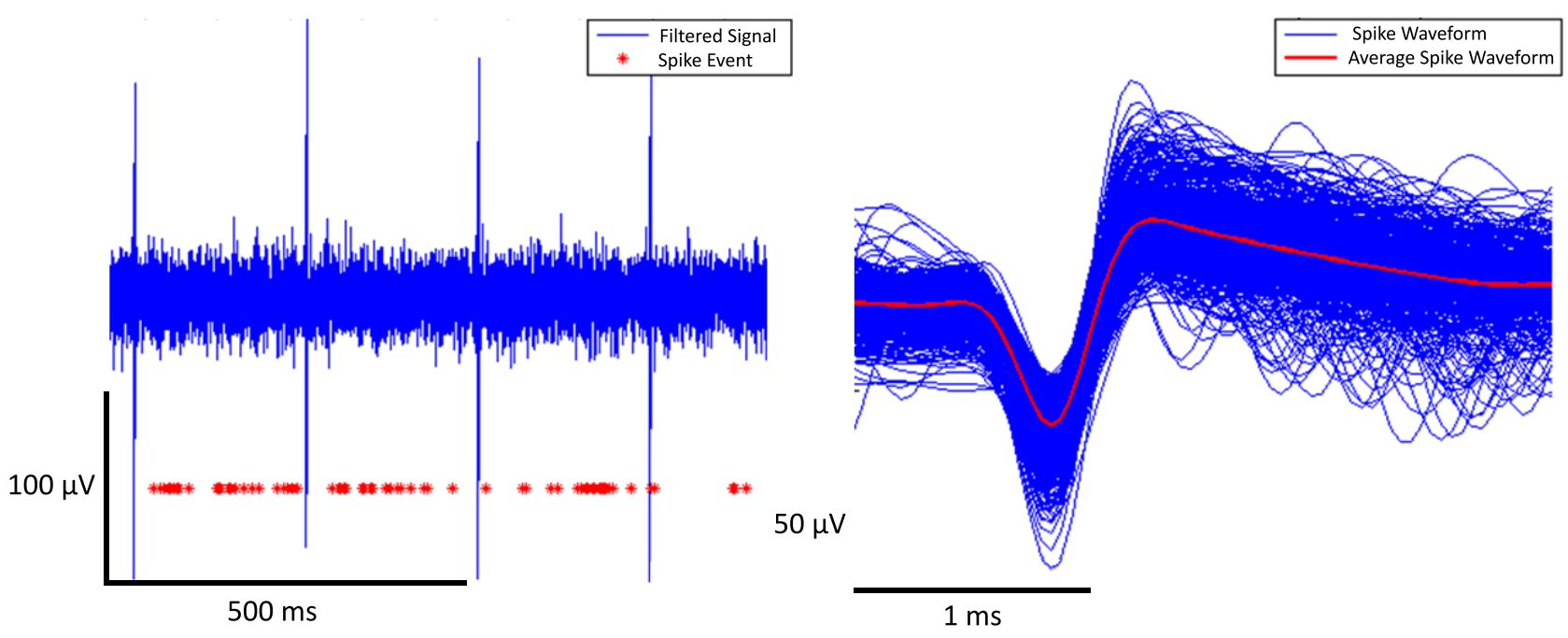

Figure 3.3: Recording of cuff electrode stimulation - Left: A short sample of the filtered neuronal data of the median nerve during electrical stimulation with a cuff electrode with a $350 \mu \mathrm{A}$ biphasic pulse. The red markings indicate the occurrences of spike events. Right: The spike waveforms of the marked unit (blue) superimposed by the average waveform (red). 


\subsubsection{Short-term implantation}

A two-week experiment was performed on a female rhesus macaque. This allowed us to explore the effects of the TIME implantation on an awake animal and to investigate the effect of the electromagnetic field on the intraneural recordings.

Two TIMEs were implanted in the median and ulnar nerve in the upper arm, while the animal was placed in balanced general anaesthesia (induction: ketamine+robinul then intubation, gas and IV analgesia). The medial nerve (N. ulnaris) of the right arm was prepared just proximal to the medial epicondylus of the elbow. The position was confirmed by electrical stimulation (50-100 $\mu \mathrm{A})$, which caused wrist and finger flexion at the radial side of the hand. The ulnar nerve (N. ulnaris) of the right arm was prepared just proximal to the medial epicondylus of the elbow. The position was confirmed by electrical stimulation $(50-100 \mu \mathrm{A})$ which caused hand ulnar flexion.

A TIME array was inserted in each the medial and the ulnar nerve. The electrodes were fixed with sutures to the thin-film electrode shaft at the distal and proximal end. Both electrode cables were tunnelled under the skin from the distal upper arm to the back of the chest (between the shoulder blade) and up to the head. Electrode connectors were embedded in the head cap and secured with a PLEXIGLAS lid.

No complications occurred during the surgery and no signs of loss of hand function were observed during the recovery period (2 days) and over the following two weeks post implantation, in which we worked with the monkey. In total 8 days of recording took place. Afterwards, the animal was sacrificed as planned for reasons unrelated to this experiment. Shortly before the perfusion, electrical stimulation was applied to the electrodes. Electrodes in the median and ulnar nerve caused flexion of the medial and lateral side of the hand, respectively. No signs of infection or rejection were observed around the implant and along the cables, which indicated that implantation was performed in a sterile fashion and that the surrounding tissue did not reject the implanted material during this period. 


\subsubsection{Intraneural recordings - First tests}

During the short term (two weeks) implantation the monkey was performing a repetitive grasping task of a handle, while the nerve activity of both the median and ulnar nerve was recorded. An example of a nerve recording during this grasping task is shown in Figure 3.4 . The recordings were found to be too noisy to clearly distinguish neural activity with the naked eye. However, the spike detection algorithm was able to find a spike-like waveform in 10 channels. The portrayed unit fired between 6-10 Hz during the arm movement phase, but most other units only fired a couple times during the whole recording. It has to be noted that in this experiment we could not determine whether the measured neural activity is related to the motor or the somatosensory pathway, due to the animal not being trained for a specific task paradigm. This requires a tighter control of the animal behaviour and the mapping of the somatosensory channels (described in Section 2.4.2), which were planned for the subsequent TIME array implantation.
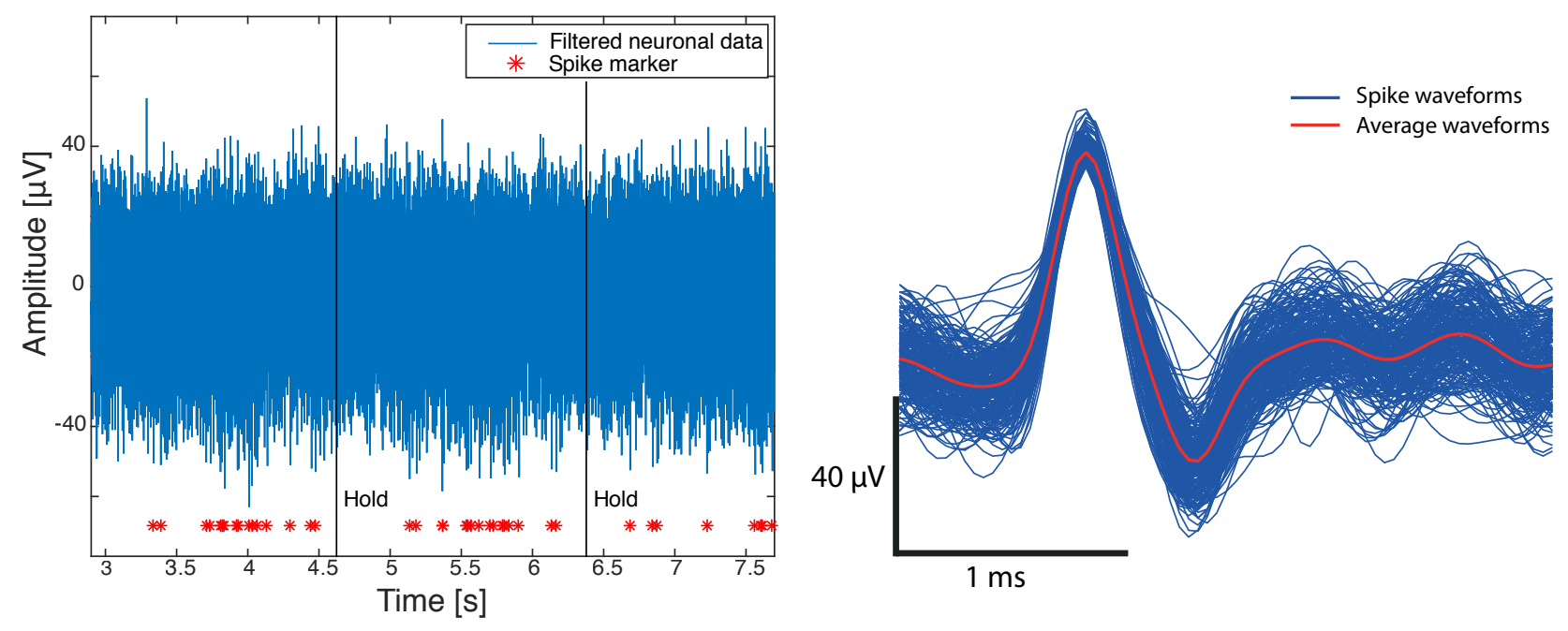

Figure 3.4: Recording Sample - Left: A short sample of the filtered neuronal data of the median nerve during the grasping of a handle. Hold marks the onset on which the handle is briefly touched. The red markings indicate the occurrences of spike events. Right: The spike waveforms of the marked unit (blue) superimposed by the average waveform (red). 


\subsubsection{Electromagnetic field influence on recording quality}

The recordings from the short term implantation also allowed us to determine if tracking of the hand kinematics is possible with TIME arrays implanted in the arm. Something that was planned for the motor decoding task at a later stage. To make a judgement, two aspects were taken into consideration: the noise levels of the recordings and ability to detect spikes. The noise levels of the background noise (defined as $5 \sigma$, see Section 2.3.4) of 4 recording pairs are displayed in a scatter plot (see Figure 3.5). Each pair consists out of two consecutive recordings of the same channel in which in one of the recordings the magnetic field was switched on in the other it was off. No consistent deviation from the diagonal was observed, indicating that the magnetic field does not substantially increase the mean noise level of the intraneural recordings (Wilcoxon Signed Rank Test, $\mathrm{p}=0.48376$ ).

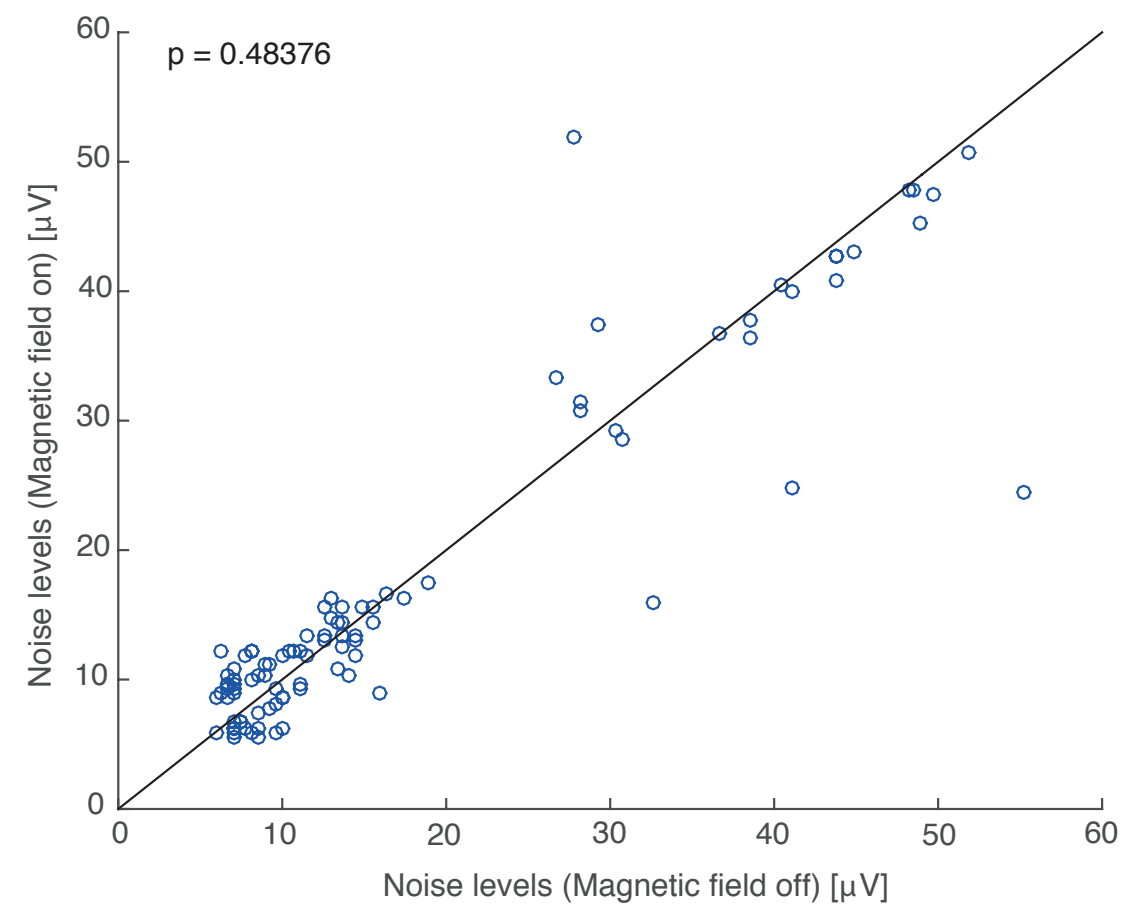

Figure 3.5: Noise level scatter plot - The noise levels (defined as the $5 \sigma$, with $\sigma=$ median $\left(\frac{|x|}{0.6745}\right)$ ) are plotted for all recording channels of four recording pairs. Each pair was recorded sequentially with the magnetic field deactivated (x-axis) and activated (y-axis). No consistent deviation from the diagonal (black) was observed (Wilcoxon Signed Rank Test, $\mathrm{p}=0.48376)$.

The influence of an electromagnetic field on the spike waveform is illustrated in Figure 3.6. Because the spike count is strongly influenced by the animal's activity and this animal was 
not trained for a specific behavioural task, it would not be correct to compare two recordings based on the amount of detected spikes in a certain time frame. Instead I compared the average shape of the waveforms of an unit. The spike detection algorithm was able to find the same unit in both recordings (each $\sim 1$ minutes long) and the spike waveform remained invariant, independent of whether the magnetic field was switched on or off. Looking at the average waveforms (see Figure 3.6) it can be seen that both the average trough amplitude and the average peak-to-trough time did not change significantly (Wilcoxon Signed Rank test, p $>0.05)$. There is a slight difference between the average waveform peak amplitudes, but this difference is minor and considering the other findings, it is safe to assume we are dealing with the same unit. These findings are promising for the ability to use our electromagnetic hand kinematics system in combination with intraneural electrode recordings.

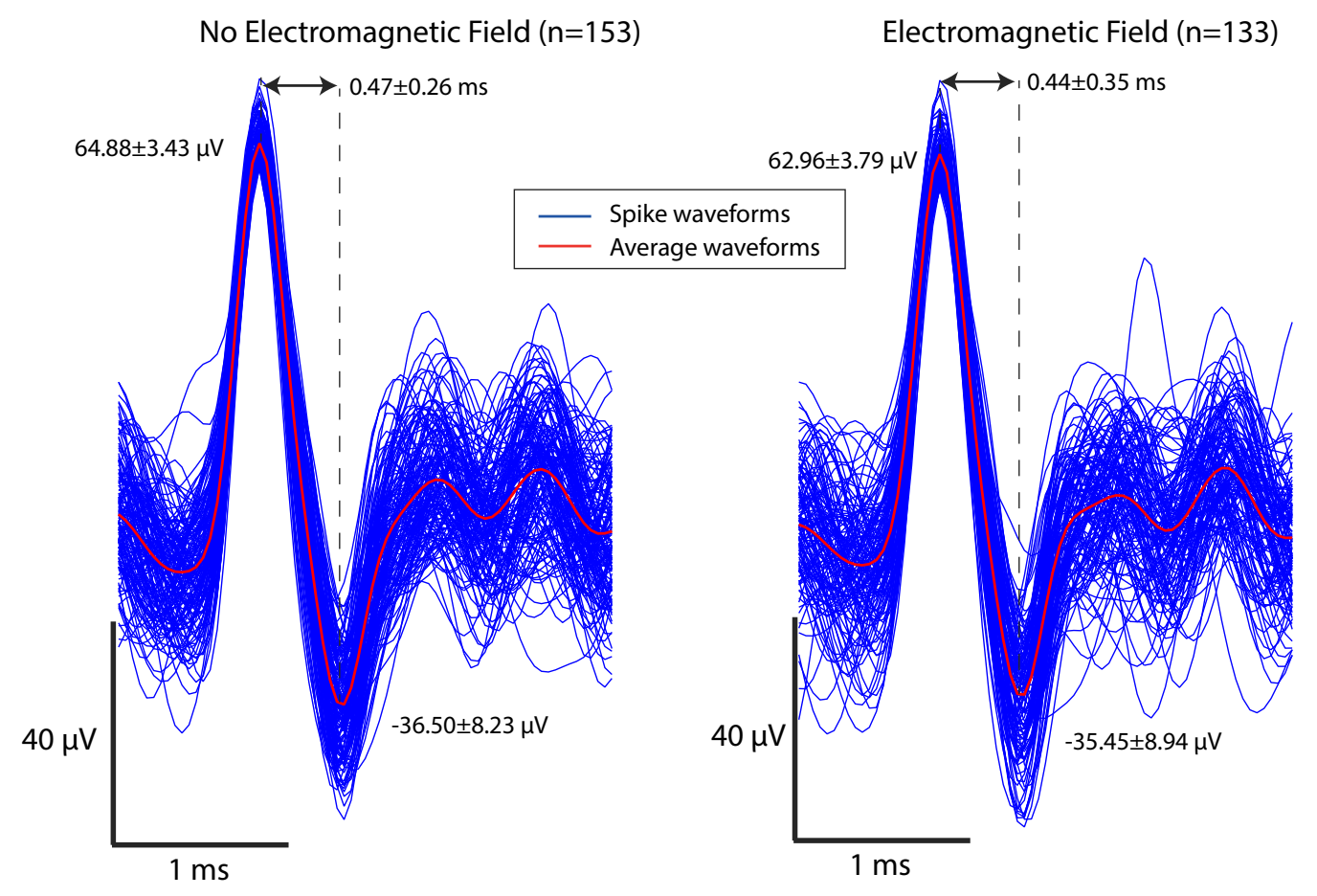

Figure 3.6: Electromagnetic field influence on spike detection - A comparison between the waveforms of the same unit detected in consecutive recordings (each $\sim 1$ minutes long) with the electromagnetic field inactive (left) and active (right). The peak and trough amplitudes, and the peak-to-trough times are indicated (mean \pm standard deviation). The presence of the electromagnetic field does not influence the trough amplitude and peak-to-trough time significantly (Wilcoxon Signed Rank Test, $\mathrm{p}=0.261$ and $\mathrm{p}=0.215$, respectively) and only a minor difference of $\sim 2 \mu \mathrm{V}$ in peak amplitude (Wilcoxon Signed Rank Test, $\mathrm{p}<<0.05$ ). Due to differences in animal behaviour, the spike count is different, but the shape of the waveform does not seem to be affected by the presence of the electromagnetic field of the hand tracking system. 


\subsubsection{Long term implantation}

Once the monkey was trained for both the motor decoding and somatosensory-discrimination task (see respectively Sections 2.3 and 2.4), it was ready for the implantation of the TIME arrays. After placing the animal in balanced general anaesthesia (induction: ketamine+robinul; intubation and gas and IV analgesia). The skin of the left arm, shoulder and neck, were shaved, cleaned and scrubbed with betadine.

Next a skin incision on the distal upper arm, just medial to the biceps muscle was made, where the median nerve (N. medianus) of the right arm just proximal to the elbow, was prepared. The nerve position was confirmed with electrical stimulation (single bipolar pulse, pulse-width $0.4 \mathrm{~ms}$, amplitude: 50-100 $\mu \mathrm{A}$ ) causing a hand radial flexion. Subsequently the ulnar nerve (N. ulnarus) of the right arm, just proximal to the medial epicondylus of the elbow, was prepared. Again this nerve position was confirmed with electrical stimulation (single bipolar pulse, pulsewidth $0.4 \mathrm{~ms}$, amplitude: 50-100 $\mu \mathrm{A}$ ), this time evoking hand ulnar flexion.

The preparation of the implantation of the TIME electrode (\#T2) in the medial nerve, started with fixating the distal end of the electrode cable to the muscular fascia using 3-0 sutures. With a needle the nerve was then punctured and with an attached thread the thin-film electrode shaft was pulled transversally through. Electrical micro-stimulation (single bipolar pulse, pulse-width $0.4 \mathrm{~ms}$, amplitude: 20-40 $\mu \mathrm{A}$ ) evoked radial wrist and finger flexion, confirming the correct placement. The distal and proximal part of the thin film was fixated at the nerve with 60 sutures and the excess part of the thin-film shaft tip was cut off. The thin-film-to-cable connector was fixated in a tissue pouch.

The EMG electrode (Cortech; 4 contact silicone patch electrode) was placed on the biceps muscle just lateral and superficial to the TIME electrode in the median nerve. There was no contact between the two electrode arrays. The distal end was fixed on the muscle fascia with 3-0 sutures and the tip of the EMG patch electrode with 6-0 sutures before encapsulating the whole in a tissue pouch. 


\subsubsection{Deplantation of TIME arrays}

The TIME arrays have a limited lifespan due to either biological rejection and/or technical failure. After the arrays cannot be used anymore for recording nor stimulation, a deplantation of the arrays occurred. The animal was placed in balanced general anesthesia (indcution: ketamine+xyalzine; intubation and gas analgesia). The skin of the left arm, shoulder and neck was shaved, cleaned and scrubbed with betadine.

The old incision, distal left upper arm just median to the biceps muscle, was opened and first the EMG silicone patch electrode was prepared. It had not only shifted from the implanted position, but also had flipped, as it was encapsulated on top of the muscle fascia with the active sites facing away from the muscle. The EMG electrode was removed by cutting the cable at the proximal margin of the incision.

With the deplantation of the TIME electrode arrays one has the choice to either remove it completely out of the nerve, or to cut the cable and leave the thin film inside. The latter allows for post-implantation examination of the TIMEs to see the effect of staying inside the body for an extended period of time. However, removing it does come with a risk of damaging the nerve. As we planned to perform another implantation on the other arm of the monkey, we chose to leave the thin film in the nerve as I did not want to risk potential paralysis of the arm.

After preparation of the median nerve, the thin film of the median nerve TIME seemed to be broken just distal of the adapter. The adapter was removed distal of the round cable and the thin film part of the electrode was left inside the nerve. Next the ulnar nerve was prepared and the thin film of this electrode seemed to be intact. However, the electrode tip was pulled out of the nerve. Like before adapter was removed and the thin film was left inside the nerve.

All that was left then was the removal of the subcutaneous cable. First the upper arm incision was closed before the animal was head fixated in prone position. After sterile re-draping an incision between the shoulder blades allowed access to the cable. These could then be pulled out of the left arm. The cable now were only attached to the connectors on the head cap. These were left intact and the cables were cut at the head cap margin on the backside of the head. 
All cables could then be removed from the back incision. Closing the back incision ended the deplantation and the animal quickly recovered without complications.

Histology After the TIME arrays of the second implantation had failed, it was decided to perform histology on the nerves to investigate the reason for array failure. After perfusion with paraffin, the implantation site was accessed through an incision in the upper arm. A distal shift of the TIME adapters was observed in both nerves. The thin-film of the median nerve TIME was still intact and still seemed to be attached to the nerve (see Figure 3.7, left). The thin-film of the ulnar TIME, on the other hand, had broken near the adapter and the TIME seemed to have been shifted slightly further out the nerve (see Figure 3.7, right).
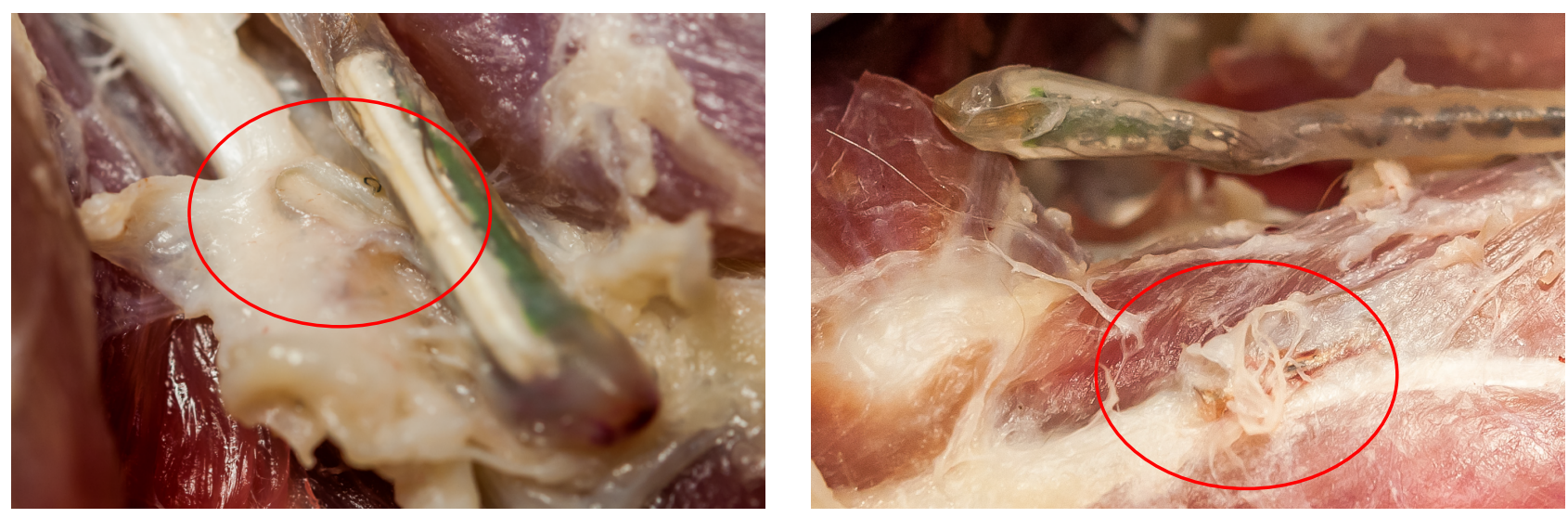

Figure 3.7: Deplantation of TIME arrays - Close-ups of the nerves during the deplantation after perfusion with paraffin. The adapters of the both TIMEs had shifted distally, either kinking the thin-film, as was observed in the median nerve (left), or breaking the thin-film near the adapter, as was observed in the ulnar nerve (right). The thin-film of both TIMEs seemed to be still attached to the nerves, but in the ulnar nerve it was shifted slightly out of the nerve. Red circles mark the thin-film location.

After the deplantation of the TIMEs, segmants of the nerves with the TIME thin-film attached, were send to the Institut de Neurociències (INc) at the Universitat Autònoma de Barcelona (UAB) where the sections were prepared by Prof. Xavier Navarro's team at the Department of Cellular Biology, Physiology and Immunology. After preparation, it was visible that the active sites were positioned mostly outside the nerve, which explains the poor electrophysiological performance that was observed during the experiments (see Figure 3.8). 


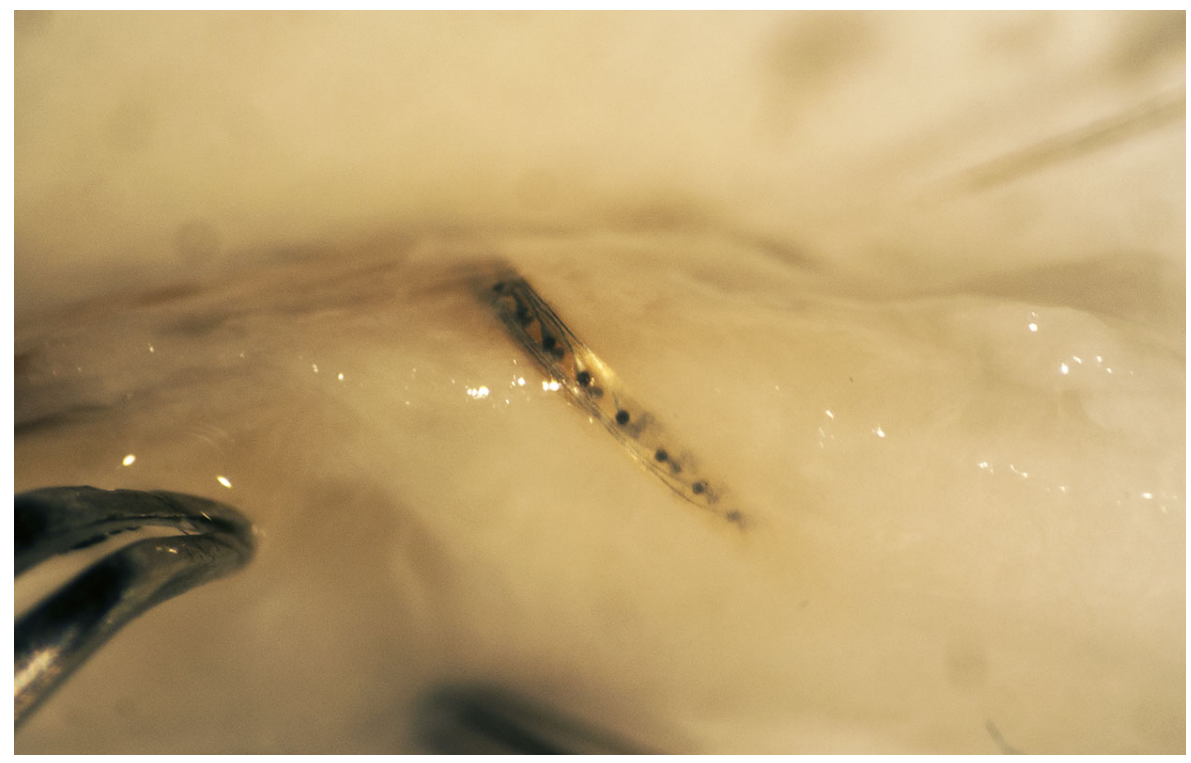

Figure 3.8: Histology TIME arrays - Preparation of the nerve confirmed the suspicion that the TIMEs had shifted in the nerve, causing (some) active sites to be located outside the nerve. 


\subsection{Motor decoding task}

\subsubsection{High noise levels and movement artefacts}

From the short term experiment it was already clear that noise would be an issue, but the recordings in the long term implantations showed how severe this problem actually was. In the raw signal not only a clear electrocardiogram (ECG) signal is present, but also strong movement artefacts caused clipping of the signal. An example is shown in Figure 3.9. The ECG signal is reasonably suppressed by the filtering steps, but the clipping was causing blank periods in the recording, which is disastrous for the signal acquisition. First of all it introduces a period in which no useful information can be acquired and secondly, since not all channels always clip simultaneously, the PCA filter then introduces new additional noise, which might be mistakenly identified as potential neural activity. The frequency spectrum shows that this noise covers a wide range of the frequency spectra.

To prevent the recordings from clipping, a $100 \mathrm{~Hz}$ HPF was applied in the recording suite. As can be seen in Figure 3.10, this cleans up the recording significantly. The ECG signal is not present anymore and the signal does not clip apart from periods in which the animal is moving very vigorously. Applying a $250 \mathrm{~Hz} \mathrm{HPF}$ can eliminate this problem even further at the cost of loss of information. Looking at he frequency spectrum, the noise cannot be specifically attributed to a specific frequency band, but is spread over the entire frequency range. Even after the excessive filtering process the movement artefacts are (though diminished) still present. No clear signs of spiking activity was seen by just looking at the filtered signal. 

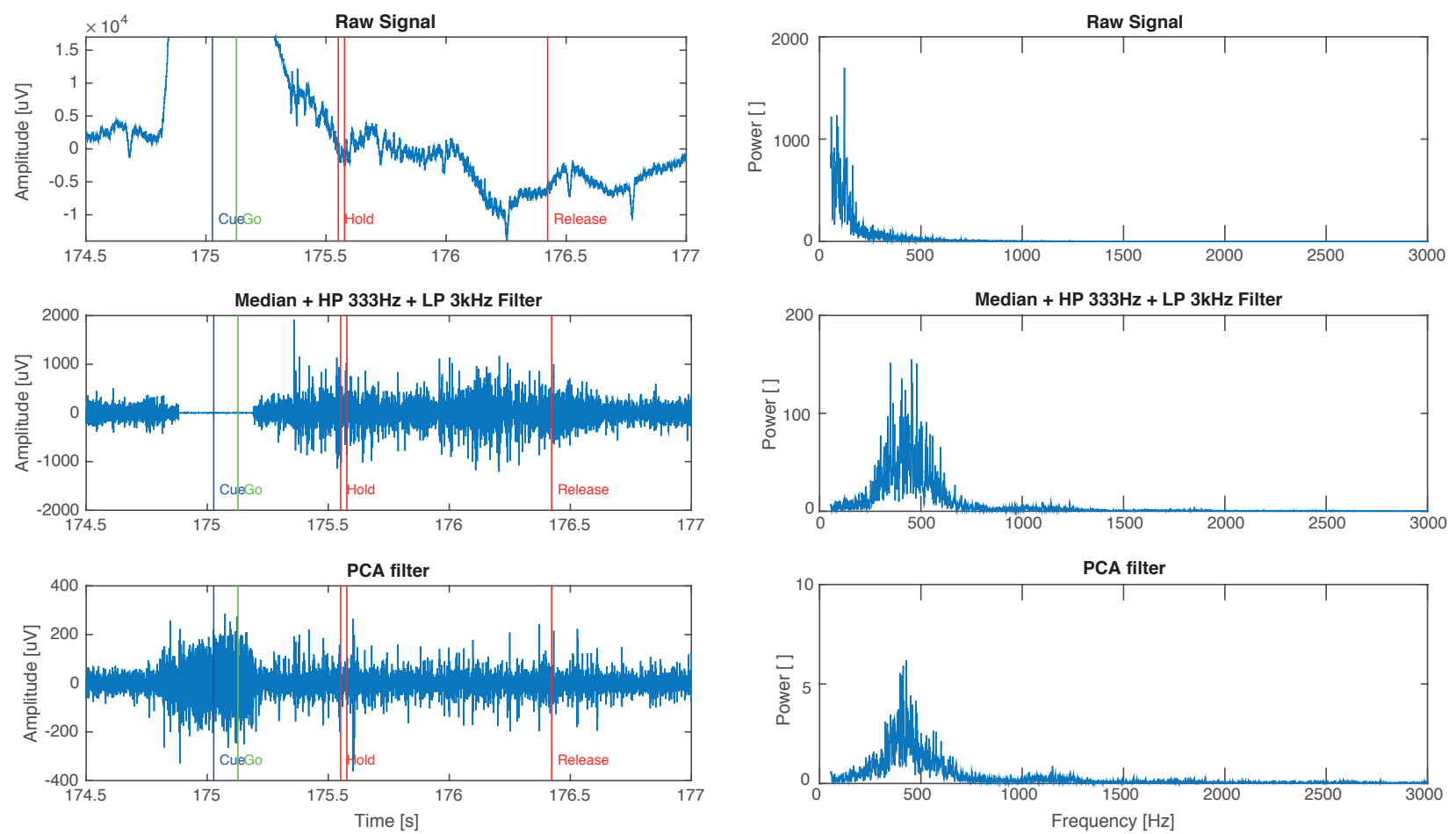

Figure 3.9: Filtering Steps (no HPF)- The effects of the filtering on the recorded neural signal in both the time domain (left column) and the frequency domain (right column). The top row shows the raw signal coming recorded by the Cerebus system (note that 0.3-7500 Hz bandpass hardware filter is still applied). The time domain shows big artefacts including clipping phases as well as an ECG signal. The frequency spectrum shows most of the information is present in the sub $500 \mathrm{~Hz}$ range. The second and third row consequently show the effects of tightening the frequency band and applying a PCA filter. This does reduce the noise, but the signal is far from noise free and the clipping phase (around the time mark of 175 seconds) causes the PCA filter to introduce noise. 

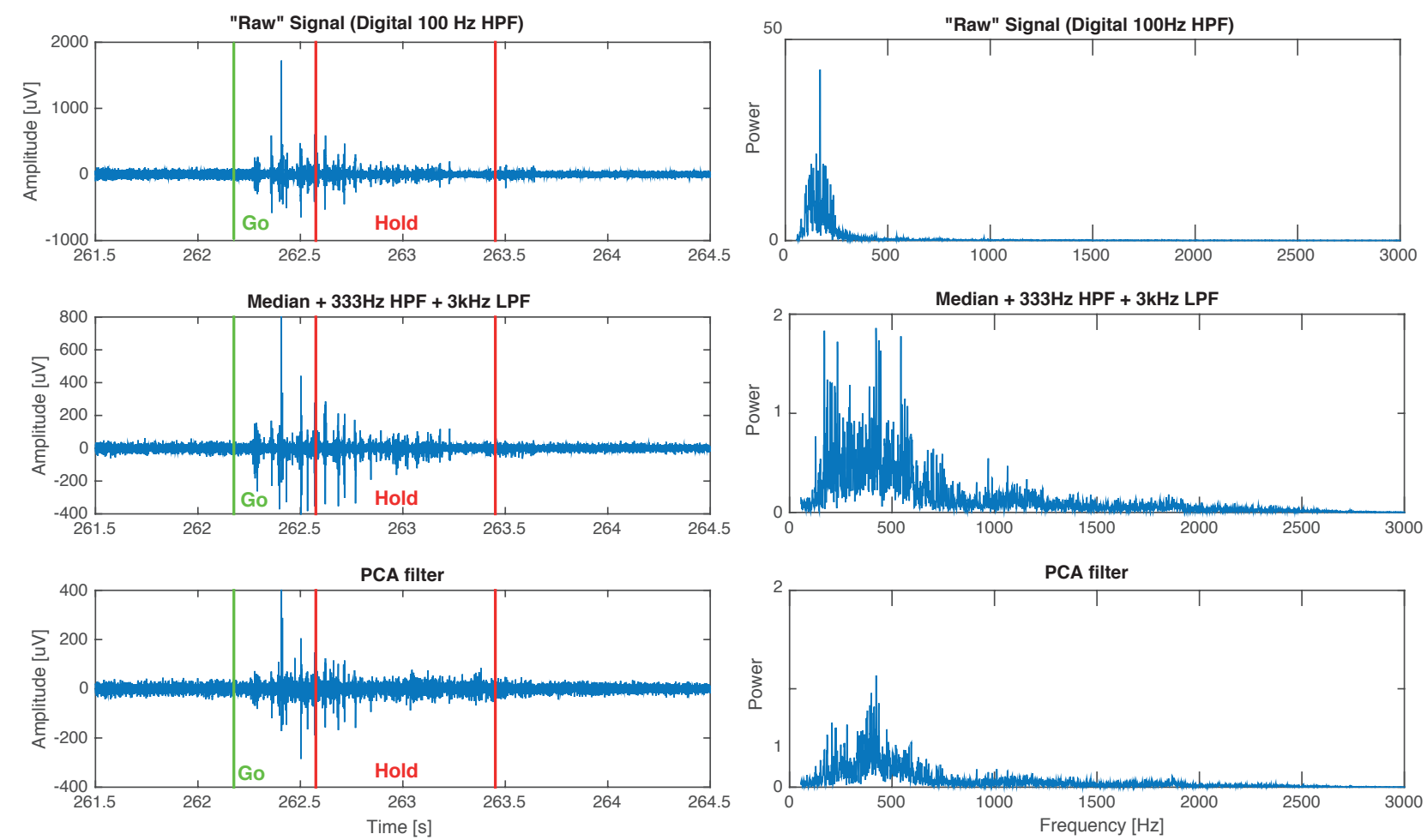

Figure 3.10: Filtering Steps (100 Hz HPF) - The effects of the filtering on the $100 \mathrm{~Hz}$ high pass filtered (HPF) recorded neural signal in both the time domain (left column) as the frequency domain (right column). The high pass filtered signal (top row) shows great improvement in noise levels and artefacts, also no clipping is observed. Tightening the frequency spectrum (middle row) reduces the noise levels further but the artefacts remain proportionally high. The PCA filter (bottom row) has a greater effect on reducing the artefacts, though the signal is still not artefact free. 


\subsubsection{Neural activity}

\section{Motor activity}

The amount of noise and artefacts present in the long-term recordings did not bode well for the spike detection process, especially since no clear spiking activity is observed, neither directly during recording nor by looking at the filtered signal (see Figure 3.10). Application of our adapted WaveClus spike detection algorithm on the data, however, did lead to the detection of some neural activity. However, in all recordings done over a period of 1,5 months, spiking activity was detected only in a single recording session. And from this recording session, only in a two channels in the median nerve a total of 3 spike waveforms were found. No neural activity was observed in the ulnar nerve. By looking at the peristimulus time histogram (PSTH) in Figure 3.11 it can be seen that the spiking activity is related to the movement of the animal as most neurons are firing the strongest during the movement (Go) epoch. On average the detected firing rate is between 2 and $13 \mathrm{~Hz}$. In the raster plot however, we can see how irregular the actual firing was across trials. For example, the raster plot for the vertical cylinder and ring grasps, we hardly detected any activity in the first couple of trials while later the unit fires repeatedly. Similar irregular firing patters are also observed for the other objects.

Despite the low number of detected units $(n=3)$, I still tried to decode the objects from the neural activity. Results from the 10-fold LDA classification are displayed in the confusion matrices in Figure 3.12. With a performance of $32.9 \%$ and $26.5 \%$ for the Go and Hold period, respectively, the performance is poor even though it was slightly above chance (16.7\%). This demonstrates the power of the applied decoding algorithms, that are able to take advantage of even small conditional differences in the recorded neurons. However, the confusion matrices demonstrate that the decoder worked indeed only for a few conditions (objects). 


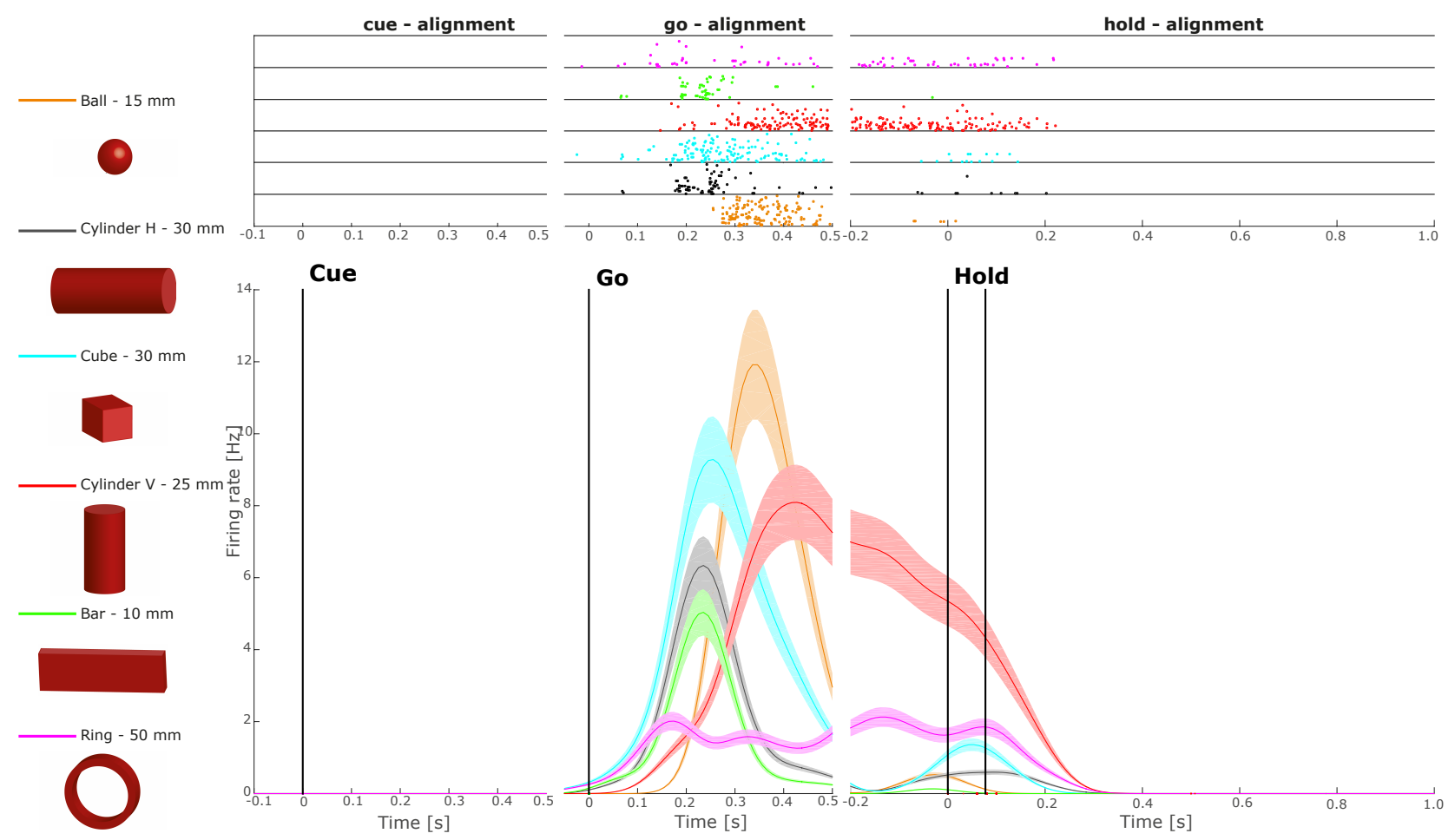

Figure 3.11: Motor Neural activity - Peristimulus time histogram (bottom) shows the average (+standard deviation) of the neural activity of a single multi-unit during the grasping task. The PSTH is split up in 3 parts, each aligned to a different trial epoch: cue, go and hold. The different colours represent which object (left) is grasped. The raster plot (top) shows the firing pattern of the multi-unit during all the trials (each line represent a single trial).
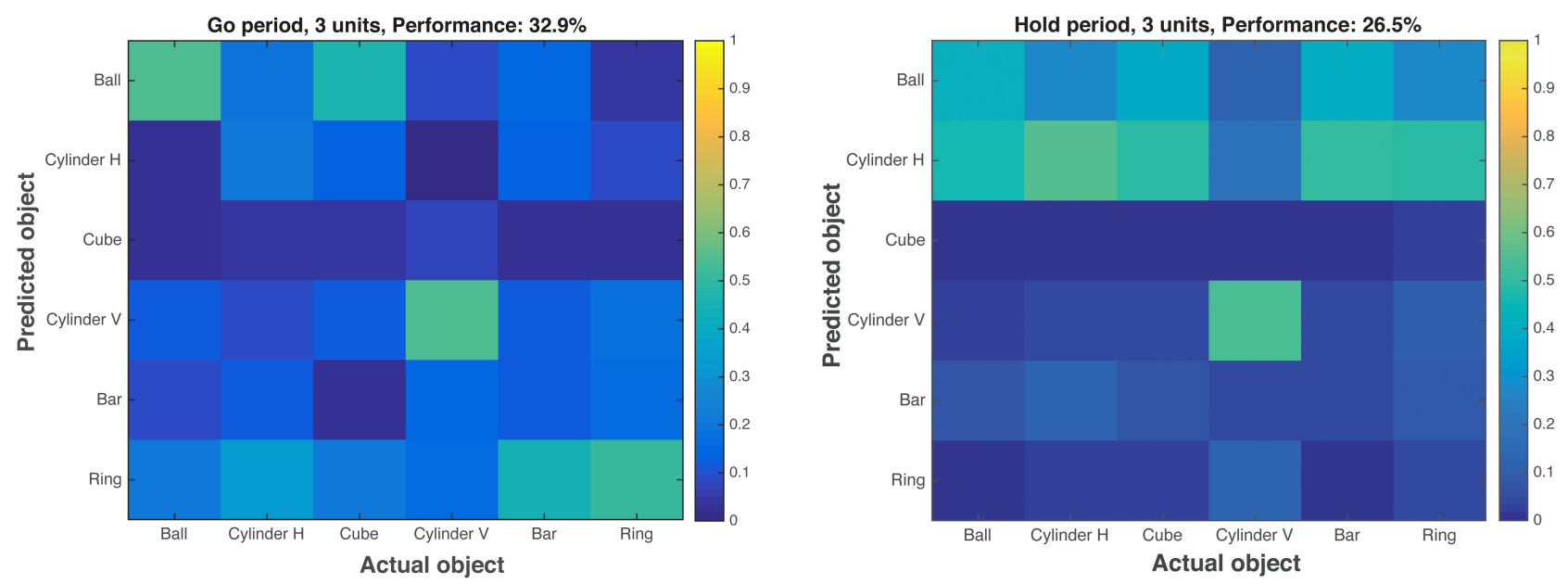

Figure 3.12: Confusion Matrices - Displays the decoding performance of the LDA decoder using the average spiking firing rate of 3 units during the Go period (left) or Hold period (right) of the trial task. The actual object is given on the $\mathrm{x}$-axis and the $\mathrm{y}$-axis portrays which object the classifier selected. The colour bar represents the decoding performance from 0 to $100 \%$. 


\section{Somatosensory activity}

Due to the excessive amount of noise present in the motor decoding recordings, we investigated if we could detect more neural activity during the somatosensory discrimination task, as movements are less pronounced in this task. Note that we stimulated the hand single sided with vibration stimulation with the highest intensity in this recording $(200 \mathrm{~Hz})$. Figure 3.13 shows that indeed the signal is less noisy, especially regarding artefacts that occur during movement. This, however, did not result in the detection of more neural activity. Again only in a few recordings, even more sparse spiking activity is detected. The PSTH in Figure 3.14 shows the activity from the detected waveform of Figure 3.15. There is no modulation of the firing rate observed during the cue phase and only a small increase during the hold phase. The other two units found in this task had similar PSTH plots. 

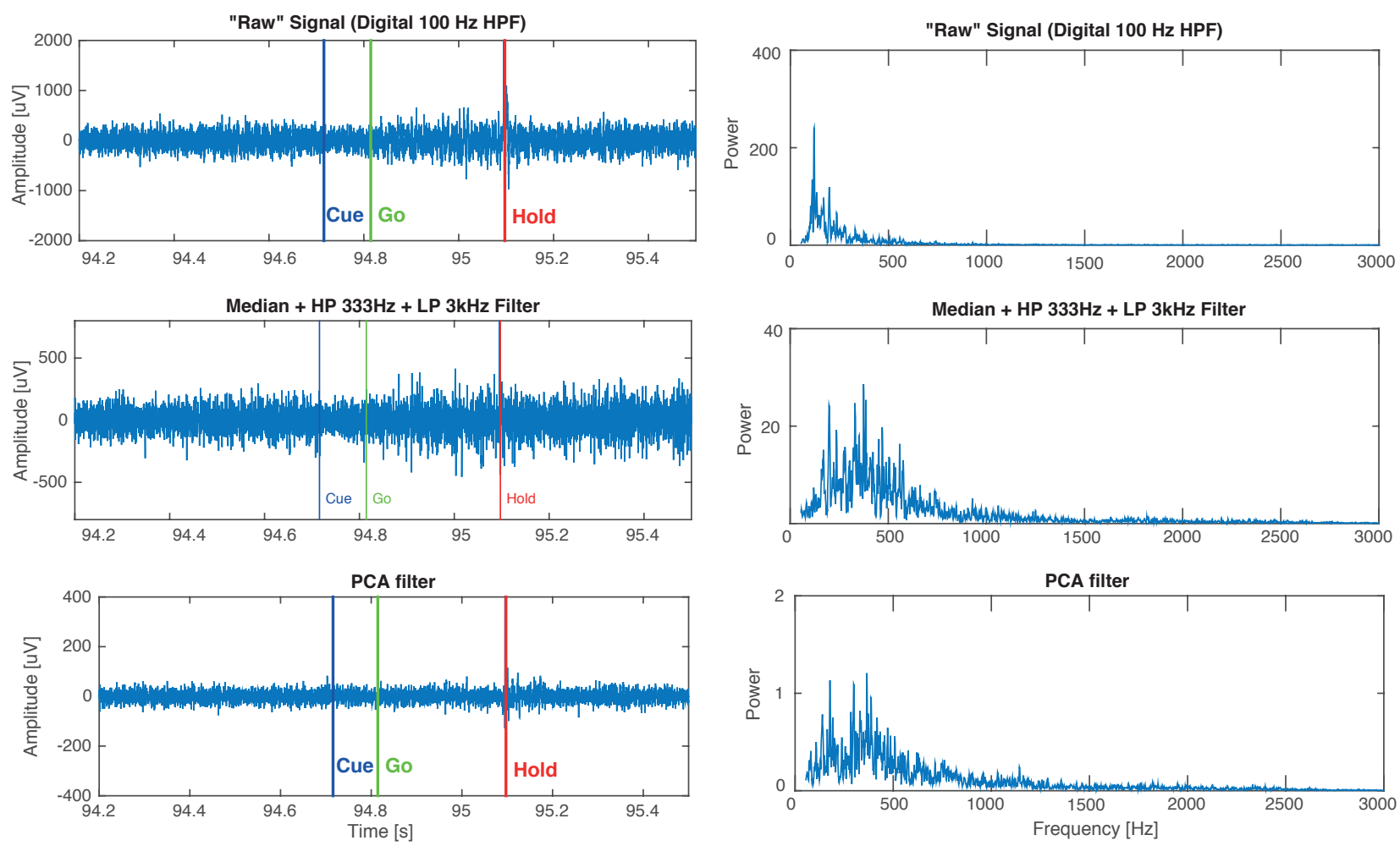

Figure 3.13: Filtering Steps, Sensory Task (100 Hz HPF) - The effects of the filtering on the $100 \mathrm{~Hz}$ high pass filtered (HPF) recorded neural signal of the somato-sensory discrimination task in both the time domain (left column) as the frequency domain (right column). Compared to the motor task recordings, the noise levels are lower and hardly any artefacts are observed. The frequency spectra also show a similar shape, indicating the same baseline noise is still present. 

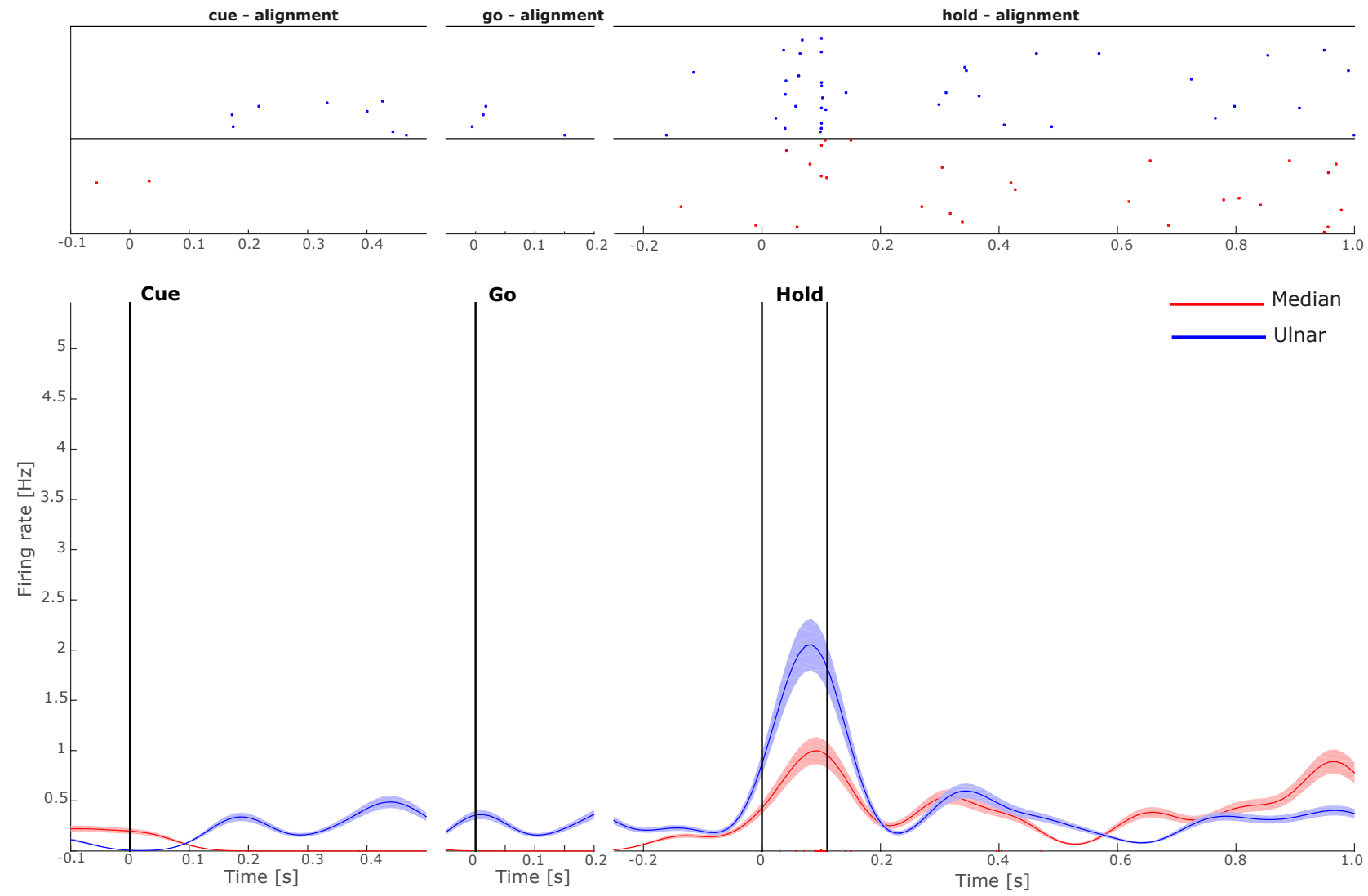

Figure 3.14: Sensory recording, PSTH - The PSTH of unit displayed in Figure 3.15 for both trials in which $200 \mathrm{~Hz}$ frequency tactile vibration was applied to either the median or ulnar side of the hand. The PSTH is aligned sequentially, from left to right, to the Cue, Go and Hold epoch of the behavioural task.

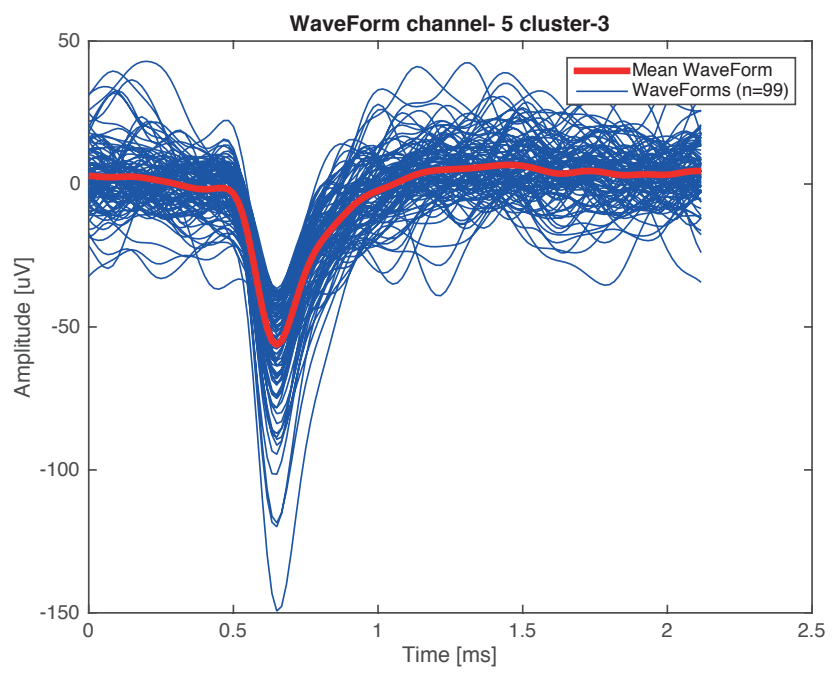

Figure 3.15: Sensory recording, Example waveform - This waveform was one of three detected waveforms during a recording of the somatosensory discrimination task. In blue the individual waveforms are plotted while in red the average waveform is superimposed. 
Since the neural information from the somatosensory recordings was too sparse, we forfeited the attempt to try and decode the stimulation sides with the LDA classifier. Instead, to see if there is any neural activity present in the signal, we looked at the power spectrum of the 800-1500 Hz band (see Figure 3.16). We analysed a recording during which the hand was simultaneously stimulated on both the median and ulnar side of the hand with tactile vibration cues. In total 6 different combinations of frequencies between 100 and $200 \mathrm{~Hz}$. In some channels a small increase is observed during the cue phase, however, this increase is not modulated by the stimulus intensity.
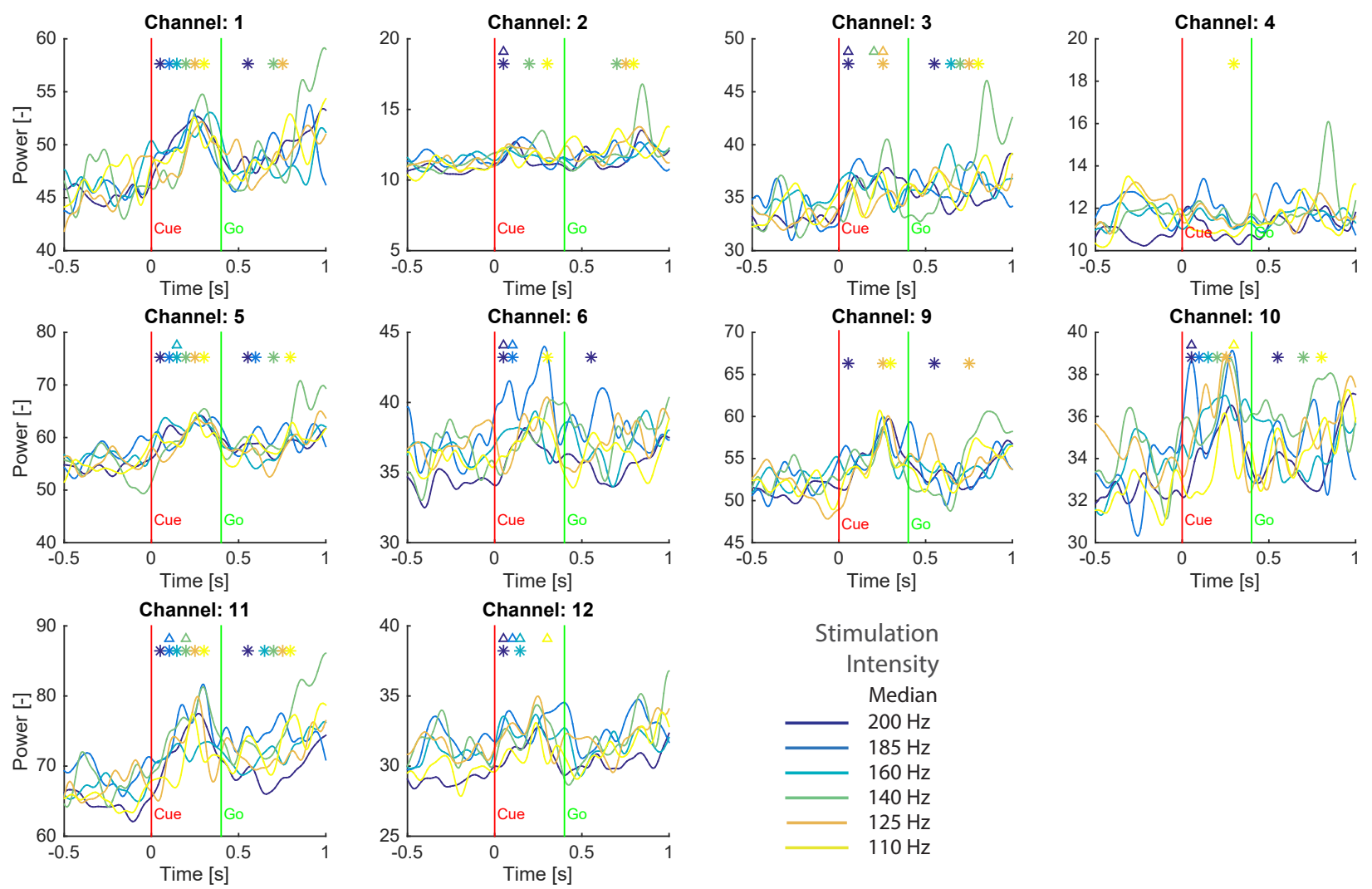

Figure 3.16: PSTH 800-1500Hz Power Band - For each channel in the median nerve TIME, the PSTH of the power of the $800-1500 \mathrm{~Hz}$ frequency spectrum is shown split up into 6 different (tactile vibration) stimulation intensities. While some channels show a significant increase in power during the cue phase, compared to the baseline in the fixation period (marked by a coloured asterisk, Wilcoxon signed-rank test, $p<0.05$ ), there is hardly any modulation differences are perceived between the stimulation intensities (marked by a coloured triangle, Wilcoxon signed-rank test, $p<0.05$,), indicating that this power change most likely cannot be attributed to the sensory stimulation. 


\subsection{Somatosensory discrimination task}

\subsubsection{Vibration only results}

The initial phase of the training with vibration discrimination took several months to complete, as this was a completely new cue task we introduced to the animal. But as soon as the animal learned to differentiate between the two vibration cues (median vs ulnar), the step to distinguish simultaneous cues was done in a matter of weeks. From here the difference between the cues was slowly decreased till the point where the animal could not distinguish it anymore (15-20 $\mathrm{Hz})$.

As a verification step during the training phase to ensure that the monkey was actually responding to the cues and not guessing the onset of the movement phase, we analysed the reaction times (see Figure 3.17). For correct trials the median reaction time is $296 \mathrm{~ms}$ (std: 98,1 ms) with a minimum of $176 \mathrm{~ms}$. For incorrect trials both the median $(275.8 \mathrm{~ms})$ reaction time is slightly lower. Note that reaction times under $100 \mathrm{~ms}$ automatically triggered an error warning. There were no indications the animal preferred stimulation on a specific side and for this specific recording the average performance lay around $80 \%$ over 324 trials (see Figure: A.5).

The performance charts in Figure 3.18 shows the animal is able to distinguish well between the tactile somatosensory stimulations applied to the hand. A gradual increase in performance is observed when the frequency difference between the median and ulnar stimulation increases. The point of discrimination (70\% Ulnar choice) lay around $20 \mathrm{~Hz}$. These results act as proof of concept that it is feasible to let a rhesus macaque indicate the relative intensity of two simultaneously applied stimulation to the hand. 


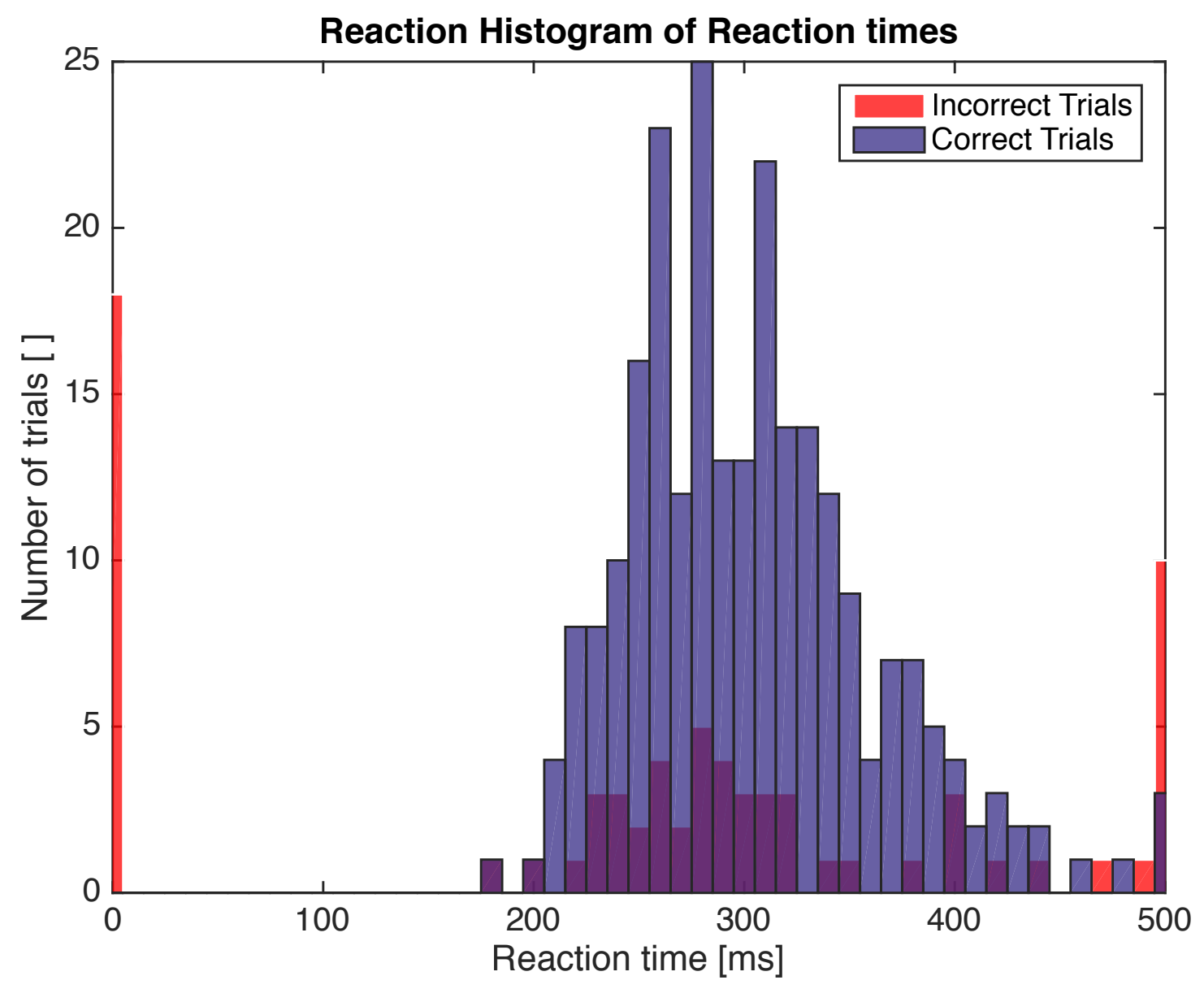

Figure 3.17: Reaction Time - Histogram of the reaction time during the somatosensory discrimination task. Reaction times are defined as the time between the end of the cue phase and the release of the hand rest button. A total of 324 trials are displayed from a single recording. Mean and standard deviation of the correct trials is $296+-98.1 \mathrm{~ms}$.

\subsubsection{Electrical stimulation only}

Due to premature electrode failure after respectively 2 months in the first implantation and 3 weeks in the second implantation, we did not have enough time to train the discrimination task with simultaneous electrical stimulation to the nerves. On a more positive note, the animal was able to differentiate between the two stimuli, just not simultaneously yet, due to insufficient available training time. 

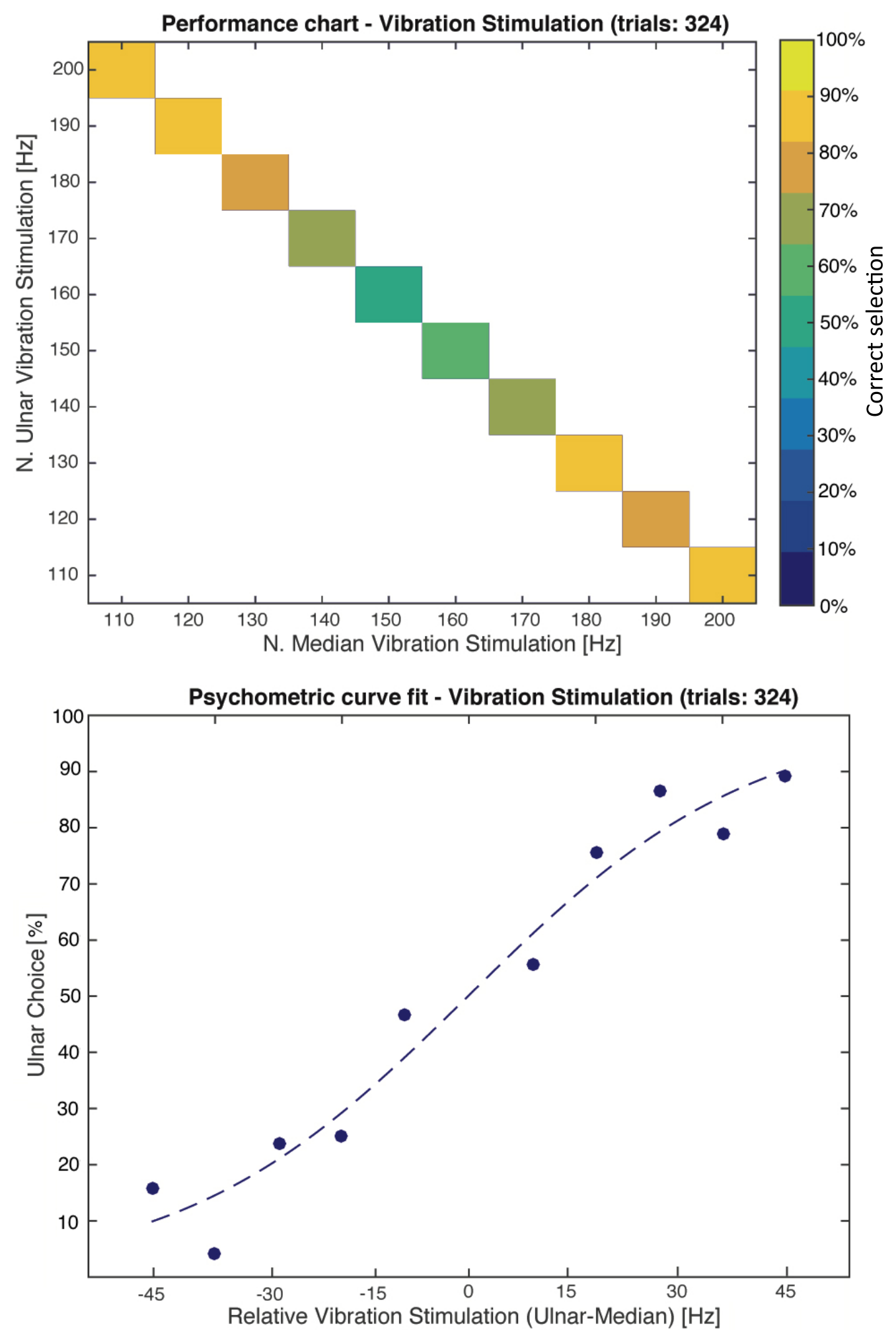

Figure 3.18: Vibration Only Results - Top: performance chart where for each stimulation pair (median vs ulnar) the percentage of correct trials is displayed. Bottom: Psychometric curve of the relative stimulation frequency (Ulnar - Median) versus the percentage of the trials the animal chose the ulnar stimulation as being more intense. The point of discrimination $(70 \%$ Ulnar choice) lays around $20 \mathrm{~Hz}$. 


\subsection{Electrode degradation}

As described in more detail in Section 2.2, the impedance and stimulation thresholds of the electrode contact sites were measured over time. In Figures 3.19 and 3.20 the impedances over time are displayed for the first and second TIME implantation, respectively. In both nerves and in both implantations we observed a slow trend towards increased impedances, though there is quite some variation from day to day. For the first implantation an average rise of 30-60 k $\Omega$ occurred over a 5 month period, while for the second implantation a similar rise was already seen after a month. In line with this finding, we see a similar trend, in the amount of functional electrode contacts, but then in a decreasing fashion. Note that for the second implantation we only monitored the impedances for little over a month, due to the premature failure of the electrodes.

Stimulation thresholds are documented in Figures 3.21 and 3.22 . The first thing to observe was the quick rise in stimulation thresholds. While intra-operatively the stimulation thresholds were in the 2-20 $\mathrm{nC}$ range, this increased almost a tenfold after 3 weeks in the first long-term implantation. This effect is apparent in both the nerves and both implantations, though in the second implantation the intra-operative thresholds already lay higher. The second trend we observed was a quick drop off channels that are able to evoke a muscle response. Looking at the median nerve in the first implantation it can be seen that after 3 weeks only two channels are functional and all stimulation capabilities were lost between the 66 and 97 day mark. In comparison, the ulnar nerve TIME still had 9 out of 12 channels functioning at this point in time. In the ulnar TIME it is seen that the stimulation threshold did not vary much after the 3 week mark, but that there is a high variability which channels are functioning. Between 142 and 160 days also this electrode array stopped working, despite the impedances still being at reasonable levels. The experiments were halted at this point. During the second implantation the amount of functional channels dropped off quicker. During the surgery the average threshold levels already lay higher and the drop off in functional channels was much more severe. Two 
weeks post-surgery the ulnar nerve could already not be stimulated anymore and 3 weeks postsurgery the same occurred in the median nerve. As the amplitude limit with the CereStim96 stimulator was reached $(210 \mu \mathrm{A})$ we moved to the A-M systems stimulator, which allowed higher amplitudes. Note that we stayed below the maximum charge (120 nC) by decreasing the phase width time. With the higher amplitudes we were able to evoke a response in the median nerve in almost all channels, but even by increasing the amplitude up to $1 \mathrm{~mA}$ no response was observed in the ulnar nerve. The experiments were halted at this time. 


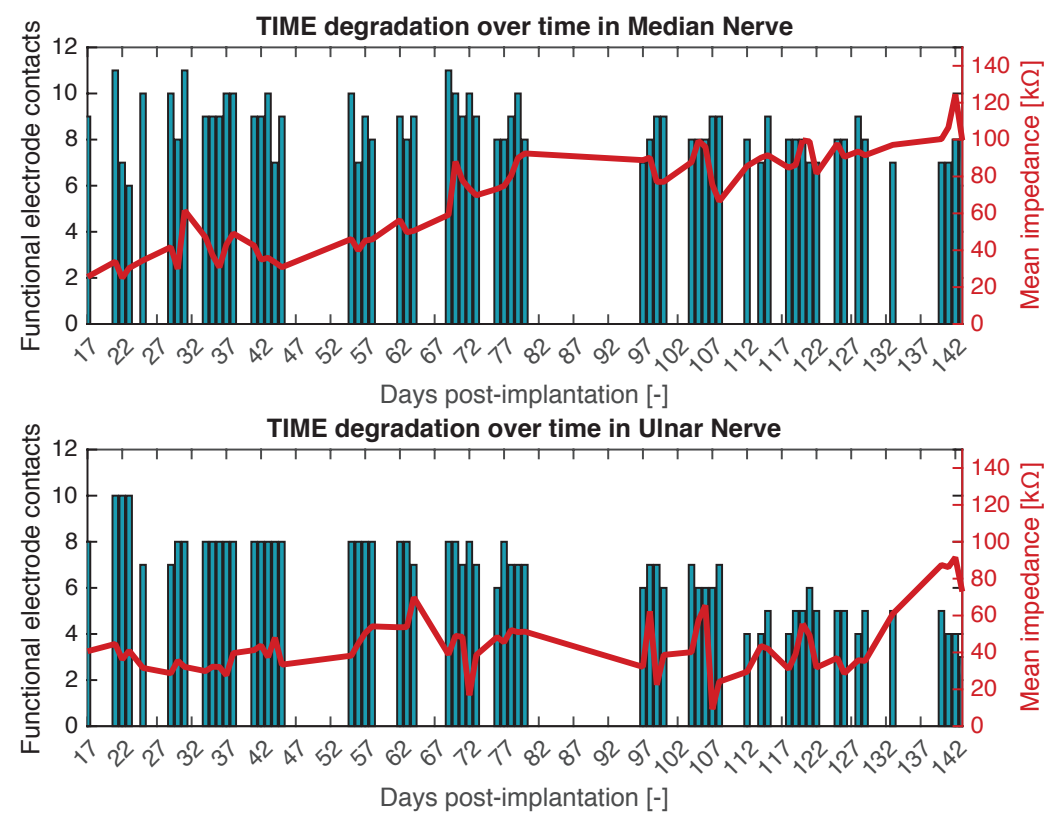

Figure 3.19: Impedances of 1st Implantation - Degradation of electrode quality (top: median nerve TIME, bottom: ulnar nerve TIME) displayed over time in terms of average impedance over all channels (red curve) as well as a histogram with the number of functional channels (A functional channel is defined as one with an impedances lower than $200 \mathrm{k} \Omega$ ). Note that the lack of blue bars indicates that there was no sample taken that day, not that the number of functional channels is zero. The data is collected over a 5 month period.
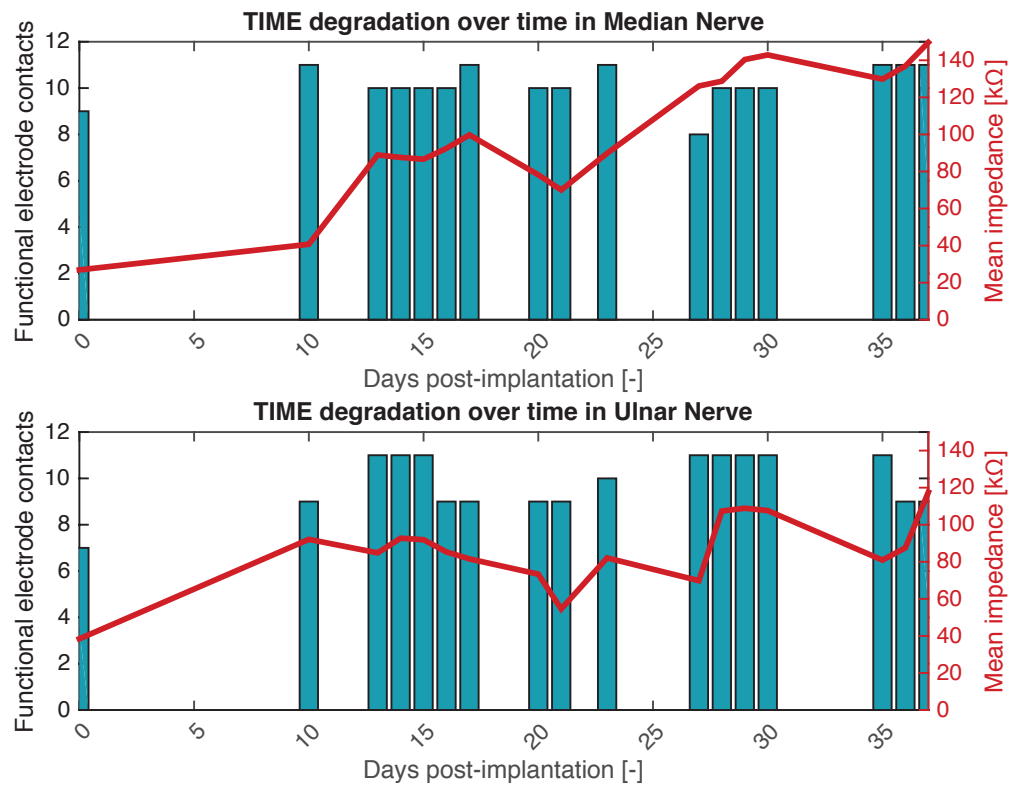

Figure 3.20: Impedances of 2nd Implantation - Degradation of electrode quality (top: median nerve TIME, bottom: ulnar nerve TIME) displayed over time in terms of average impedance over all channels (red curve) as well as a histogram with the number of functional channels (A functional channel is defined as one with an impedances lower than $200 \mathrm{k} \Omega$ ). Note that the lack of blue bars indicates that there was no sample taken that day, not that the number of functional channels is zero. The data is collected over a 1 month period. 


Median nerve
\begin{tabular}{l|l|l|l|l|l|l|l|l|l} 
Channel & $\begin{array}{l}\mathbf{0} \\
\text { Days }\end{array}$ & $\begin{array}{l}\mathbf{2 2} \\
\text { Days }\end{array}$ & $\begin{array}{l}\mathbf{5 2} \\
\text { Days }\end{array}$ & $\begin{array}{l}\mathbf{6 3} \\
\text { Days }\end{array}$ & $\begin{array}{l}\mathbf{6 6} \\
\text { Days }\end{array}$ & $\begin{array}{l}\mathbf{9 7} \\
\text { Days }\end{array}$ & $\begin{array}{l}\mathbf{1 2 6} \\
\text { Days }\end{array}$ & $\begin{array}{l}\mathbf{1 4 2} \\
\text { Days }\end{array}$ & $\begin{array}{l}\mathbf{1 6 0} \\
\text { Days }\end{array}$ \\
\hline R1 & $8 \mathrm{nC}$ & - & - & - & - & - & - & - & - \\
\hline $\mathrm{R} 2$ & - & - & - & - & - & - & - & - & - \\
\hline $\mathrm{R} 3$ & $8 \mathrm{nC}$ & - & - & - & - & - & - & - & - \\
\hline $\mathrm{R} 4$ & - & $75 \mathrm{nC}$ & - & - & - & - & - & - & - \\
\hline $\mathrm{R} 5$ & $4 \mathrm{nC}$ & - & - & - & - & - & - & - & - \\
\hline $\mathrm{R} 6$ & $12 \mathrm{nC}$ & - & - & - & - & - & - & - & - \\
\hline $\mathrm{L} 1$ & $8 \mathrm{nC}$ & - & - & - & - & - & - & - & - \\
\hline $\mathrm{L} 2$ & $8 \mathrm{nC}$ & - & - & - & - & - & - & - & - \\
\hline $\mathrm{L} 3$ & $4 \mathrm{nC}$ & - & - & - & - & - & - & - & - \\
\hline $\mathrm{L} 4$ & $4 \mathrm{nC}$ & - & - & - & - & - & - & - & - \\
\hline $\mathrm{L} 5$ & $4 \mathrm{nC}$ & $50 \mathrm{nC}$ & - & - & - & - & - & - & - \\
\hline $\mathrm{L} 6$ & $4 \mathrm{nC}$ & - & $70 \mathrm{nC}$ & $45 \mathrm{nC}$ & $75 \mathrm{nC}$ & - & - & - & -
\end{tabular}

Ulnar nerve
\begin{tabular}{l|l|l|l|l|l|l|l|l|l}
\multirow{2}{*}{ Channel } & $\begin{array}{l}\mathbf{0} \\
\text { Days }\end{array}$ & $\begin{array}{l}\mathbf{2 2} \\
\text { Days }\end{array}$ & $\begin{array}{l}\mathbf{5 2} \\
\text { Days }\end{array}$ & $\begin{array}{l}\mathbf{6 3} \\
\text { Days }\end{array}$ & $\begin{array}{l}\mathbf{6 6} \\
\text { Days }\end{array}$ & $\begin{array}{l}\mathbf{9 7} \\
\text { Days }\end{array}$ & $\begin{array}{l}\mathbf{1 2 6} \\
\text { Days }\end{array}$ & $\begin{array}{l}\mathbf{1 4 2} \\
\text { Days }\end{array}$ & $\begin{array}{l}\mathbf{1 6 0} \\
\text { Days }\end{array}$ \\
\hline R1 & - & - & $90 n C$ & $70 n C$ & - & $70 n C$ & - & - & - \\
\hline R2 & - & - & $90 n C$ & - & - & $60 n C$ & - & - & - \\
\hline R3 & - & - & $90 n C$ & $50 n C$ & - & $50 n C$ & - & - & - \\
\hline R4 & $4 n C$ & - & $90 n C$ & - & - & $95 n C$ & - & - & - \\
\hline R5 & $8 n C$ & - & $90 n C$ & $50 n C$ & - & $50 n C$ & - & - & - \\
\hline R6 & - & $100 n C$ & $70 n C$ & - & - & $80 n C$ & - & - & - \\
\hline L1 & $20 n C$ & - & - & - & - & - & $90 n C$ & - & - \\
\hline L2 & $8 n C$ & - & $90 n C$ & - & $90 n C$ & - & $80 n C$ & $65 n C$ & - \\
\hline L3 & $4 n C$ & - & - & - & - & - & $80 n C$ & - & - \\
\hline L4 & $12 n C$ & - & $90 n C$ & - & - & $80 n C$ & $95 n C$ & $70 n C$ & - \\
\hline L5 & $16 n C$ & $100 n C$ & $90 n C$ & - & $80 n C$ & $55 n C$ & $75 n C$ & $60 n C$ & - \\
\hline L6 & - & $100 n C$ & $90 n C$ & - & $80 n C$ & $75 n C$ & - & $90 n C$ & -
\end{tabular}

Figure 3.21: Stimulation Thresholds 1st Implantation - The stimulation threshold (defined as the minimum charge needed to evoke a muscle twitch) over time for every TIME contact sites for the median nerve (left) and ulnar nerve (right) TIME electrode array. Empty non-coloured entries indicate that no response could be evoked.

\begin{tabular}{|c|c|c|c|c|c|}
\hline Channel & \begin{tabular}{|l}
0 \\
Days
\end{tabular} & \begin{tabular}{|l}
20 \\
Days
\end{tabular} & \begin{tabular}{|l|}
21 \\
Days
\end{tabular} & \begin{tabular}{|l}
23 \\
Days
\end{tabular} & \begin{tabular}{|l|}
37 \\
Days
\end{tabular} \\
\hline R1 & - & - & - & $62,5 \mathrm{nC}^{*}$ & - \\
\hline R2 & $45 \mathrm{nC}$ & - & - & $62,5 n C^{*}$ & - \\
\hline R3 & - & - & - & $62,5 \mathrm{nC}^{*}$ & - \\
\hline R4 & $12,5 \mathrm{nC}$ & - & - & $62,5 \mathrm{nC}^{*}$ & - \\
\hline R5 & $30 \mathrm{nC}$ & - & - & $62,5 \mathrm{nC}^{*}$ & - \\
\hline R6 & - & - & - & $62,5 \mathrm{nC}^{*}$ & - \\
\hline L1 & - & - & - & $62,5 n C^{*}$ & - \\
\hline L2 & $40 \mathrm{nC}$ & - & - & $62,5 n C^{*}$ & - \\
\hline L3 & $17,5 \mathrm{nC}$ & $90 \mathrm{nC}$ & $80 \mathrm{nC}$ & $62,5 \mathrm{nC} *$ & - \\
\hline L4 & - & - & - & $62,5 n C^{*}$ & - \\
\hline L5 & $30 \mathrm{nC}$ & - & - & $62,5 \mathrm{nC}^{*}$ & - \\
\hline L6 & $40 \mathrm{nC}$ & $60 \mathrm{nC}$ & $55 \mathrm{nC}$ & $62,5 \mathrm{nC}^{*}$ & - \\
\hline
\end{tabular}

Ulnar nerve
\begin{tabular}{l|l|l|l|l|l} 
Channel & 0 Days & $\begin{array}{l}\mathbf{2 0} \\
\text { Days }\end{array}$ & $\begin{array}{l}\mathbf{2 1} \\
\text { Days }\end{array}$ & $\begin{array}{l}\mathbf{2 3} \\
\text { Days }\end{array}$ & $\begin{array}{l}\mathbf{3 7} \\
\text { Days }\end{array}$ \\
\hline R1 & $2,5 \mathrm{nC}$ & - & - & - & - \\
\hline R2 & $2,5 \mathrm{nC}$ & - & - & - & - \\
\hline R3 & - & - & - & - & - \\
\hline R4 & - & - & - & - & - \\
\hline R5 & - & - & - & - & - \\
\hline R6 & - & - & - & - & - \\
\hline L1 & - & - & - & - & - \\
\hline L2 & - & - & - & - & - \\
\hline L3 & $2,5 \mathrm{nC}$ & - & - & - & - \\
\hline L4 & $125 \mathrm{nC}$ & - & - & - & - \\
\hline L5 & - & - & - & - & - \\
\hline L6 & - & - & - & - & -
\end{tabular}

Figure 3.22: Stimulation Thresholds 2nd Implantation - The stimulation threshold (defined as the minimum charge needed to evoke a muscle twitch) over time for every TIME contact sites for the median nerve (left) and ulnar nerve (right) TIME electrode array. Empty non-coloured entries indicate that no response could be evoked. Note that lighter green entries indicate that a different stimulator was used (A-M Systems) that allowed higher amplitudes, in order to see if that would evoke a muscle response, which the lower amplitude pulses from the CereStim96 was unable to elicit. 



\section{Chapter 4}

\section{Discussion}

\subsection{Implantation techniques}

\subsubsection{General aspects}

In total 5 PNS implantations have occurred in which we tested the feasibility of implanting PNS interfaces in the median and ulnar nerve. The first two were terminal and explorative, and gave us enough confidence to perform long(er)-term implantations. The fact that we did not see any signs of paralysis or movement inhibition (asides from temporary discomfort caused by skin lesion and stitches) during the 3 consecutive long-term non-terminal implantations shows that the implantation technique is established, at least in regards towards the potential health risks it carries.

This does not mean the implantation technique is flawless. The biggest limitation is that as surgeon you have no control over which fascicles you penetrate. Once the needle penetrates the nerve, one does not know how many, nor which fascicles are penetrated, if any at all. To increase the chance of hitting a fascicle, several TIME electrodes would have to be inserted randomly in the nerve. Even if there was a way to visualise if the array is situated in or near fascicle, one still would not know where these fascicles are connected to. They might innervate the hand, but since the implantation occurs in the upper arm, they might as well innervate a 
part of the skin in the lower arm instead.

The surgeon's only tool to rely on is a topographic map of the nerves, but unfortunately there are no exact maps. Sunderland, 1945 was one of the first who tried to make a generalised map of the nerves, but not until recently this was explored further by Delgado-Martínez et al., 2016]. They showed that making a generalised map overlapping the nerve branching topography is not possible: There is too much individual variation in the nerve branching structure among people, and factors like fascicle size and distance to the nerve's center are not determining their innervation location. Mapping the nerves fascicle structure of amputee patients beforehand is also not feasible, as the fascicles cannot be traced to their end/start points. And coming back to our surgeon problem, even in the case of mapping possibilities, the surgeon does not have proper means to verify the orientation of the nerve intra-surgically, as methods such as stereotaxy and MRI imaging - used for mapping the CNS - are not applicable for the PNS Scherberger et al., 2003.

What we can work with is branching probability, that is the likelyhood that the fascicle you want to interface with has not branched off at certain location allong the nerve. DelgadoMartínez et al., 2016] showed that fascicles tend to only move distal in the nerve briefly before branching off. So in the upper arm the fascicles that innervate the hand are most likely still positioned centrally in the nerve. Another finding is that only below the antecubial fossa (a landmark near the elbow) the topography starts to be more defined, as most of the upper arm nerves have branched away at this point. For motor decoding with TIME electrodes this would be the most suitable location, because first of all you are more likely to interact with lower arm muscles that control the hand at this location. Second, because of the transverse implantation orientation of the TIME you are able to reach the fascicles around the nerve axis, which more likely innervate distal arm/hand locations. However, this is also a site with little internal space to implant such a nerve interface and movement of the elbow joint would put considerable stress on the electrodes. For this reason it was decided to implant the TIMEs in the upper arm, but as distally as possible.

A suitable implantation location for somatosensory electrical stimulation, a very distal location 
of the lower arm would be most suitable. Most motor fascicles have branched away and mostly sensory fascicles remain. The nerves do become increasingly smaller here, so shifting to a cuff electrode might be an option to consider. Cuff electrodes also have the advantage of being more biocompatible and since the nerve does not contain a high number of fascicles anymore at this location, the low selectivity of cuff electrodes does not weigh as gravely. As motor decoding was our primary objective and implanting TIMEs in both the upper and lower arm was not an option because of the increased risk, we opted to stay with a single TIME array in each nerve in the upper arm for both nerve recording and stimulation.

A final point to note regarding the implantation location is that these are ideal implantation locations, which circumstances will not always allow. For example, for a trans-humeral amputee patient (above the elbow) the best option is to implant it as close to the stump as possible.

\subsubsection{Non-human primate aspects}

Since we are working with the rhesus macaque model, several differences have to be taken into account regarding the investigation of PNS interfaces for bi-directional prosthetic control. First of all, while the macaque serves as a good model for human grasping, we do have to be aware of the morphological and behavioural differences. With respect to the morpohological difference, the most obvious one is found in the elbow-shoulder posture and the underdevelopment of the thumb Christel and Billard, 2002]. The behavioural differences have an effect on surgical recovery period, during which the animal is more prone to opening the wound margins, and it influences ability to train specific tasks. Since rhesus macaque are able to perform fine finger movements and are very sensitive in their hands like us humans, the designs of both the motor decoding and somatosensory tasks are suitable achievable.

A second aspect to take into account with working with rhesus macaques is the difference in body size, and thus the difference in nerve size, as compared to humans. These nerves are considerably smaller than human nerves ( factor 2 in diameter), which complicates the TIME implantation. Not only are they more difficult to handle intra-surgically, the size can also cause the electrode contacts to stick out, as we have observed in some of our implantations. With the 
larger human nerves the latter should not pose such a big problem, though one has to consider there is still a big variety in nerves between age, sex and other anthropometric factors Stetson et al., 1992.

The smaller size of the nerves also disabled us to perform interfascicular nerve splitting by opening the epineurium and then threading the TIME through individual fascicles. While this does insure penetration of the fascicles, instead of moving in between them, it does not come without a risk. The nerves are delicate structures and need to be handled carefully. While this method is applied with success on human patients Raspopovic et al., 2014 we chose not to apply it. In our experiments this was not an option since a) the monkey's nerves are very small, and b) the risk of nerve damage would be too great. The latter is not much smaller in human patients, but the potential loss of hand function for an amputee patient is self-explanatory a non-issue. In the animal experiments a paralysis would mean not being able to perform the experiment. Even if the paralysis would only be temporarily, we would lose weeks of investigation time. Since time between the implantation and the TIME array failure is already so limited, this risk was not worth taking.

\subsection{Recording quality}

The recordings made during both experimental tasks showed it is challenging to deal with the noise in the signal. With every movement the animal made clipping was observed, which is disastrous for the signal acquisition (as shown in Figure 3.9). Once the amplifier clips one loses all information during this period. Passing the signal through a $100 \mathrm{~Hz} \mathrm{HPF}$ before recording cleans up the signal considerably. The ECG signal is suppressed and only in a few cases clipping was observed. This step is crucial for enabling data acquisition during the movement phase. A $250 \mathrm{~Hz}$ HPF filter does eliminate the clipping completely, but one has to keep in mind that it does come at a cost: the loss of information. The stricter the data is filtered, the more difficult the spike sorting process becomes, as the fine shape information of the waveforms of individual spike units is lost. The $100 \mathrm{~Hz}$ HPF also disabled the ability to analyse potential low frequency potentials (LFP), which could have yielded useful information. The reason I justified applying 
the $100 \mathrm{~Hz} \mathrm{HPF}$ is that at this stage of the experiment, it was more important to get any kind of neural information out of the recordings than to worry if a multi-unit is identified as single unit. Also when the signal is clipping, all the information is lost, so LFP analysis would then also not been possible either.

The absence of any visible spiking activity with the naked eye during the recording, even after filtering, did not bode well for the spike detection. After the actual spike detection and spike sorting process, this suspicion was confirmed. The first couple of recordings yielded no neural activity at all. Many things were tried to improve the signal quality but to no avail. From switching the 16pin-to-36-pin Omnetics adapters, from a wire to a PCB type, did not have any effect. Neither did flipping the connectors around (in case a defect reference channel was at fault), nor enabling/disabling the common ground to the metal head post. Only switching on the HPF filter helped somewhat to clean the signal. In the end spike waveforms were detected only in a single recording of the motor task. But the amount of neural activity was very sparse and too low to decode the grip type reliably.

It was hoped that the somatosensory recordings would yield better results, as there is hardly any movement that could introduce noise to the signal. However, again only in a single recording we found (even more sparse) neural activity. While this could be attributed to the fact that these recordings took place 1,5 months post implantation, which is fairly late considering that the optimal recording quality is achieved within the first month after implantation. It is actually more likely that there was no somatosensory activity picked up by the array at all. In the PSTH we only observed a small activity bump in the hold phase, which might as well be attributed to the muscle activity of the pressing movement. Also the power spectrum of the $800-1500 \mathrm{~Hz}$ band did not show significant modulation in in the cue phase, proving the absence of neural activity. Even if the recording took place earlier in time, the findings from the motor recordings already showed that there was hardly any neural activity to begin with. This and the fact that the upper arm contains relatively little sensory fascicles, makes the chance small that we would detect sensory only activity.

A clear explanation, why so little neural activity was found during the motor recordings, cannot 
be given. A possible cause is that we are not penetrating the fascicles of the nerve with the TIME, but instead recording from the insulating tissue between the fascicles. This diminishes the signal strength severely and in combination with a low signal-to-noise ratio, the neural signal is washed out by the environment noise. A possible explanation for why some neural activity was detected for a brief moment, could be that sporadic micro shifting of the array moved an active site briefly close enough to a fascicle to detect some activity within it. However, prior to having histology results available we cannot verify this theory.

When comparing my methodology with recordings done in humans and rodents, which were more successful in detecting neural activity, there are two differences that could attribute to the poor signal-to-noise ratio. First, the length of the subcuteanous electrode wire: The weak neural signals have to travel unamplified through $50 \mathrm{~cm}$ of wire and subsequently pass through another adapter before it goes into the headstage, where it is digitalised and amplified. This makes the signal very susceptible to environment noise, especially considering it runs past many muscles and close to the heart. A second reason is that we are conducting the experiment in a task which encompasses movement of the arm. The human experiments worked with an amputee patient, which did not move during the recordings. Also in that case the electrode wire came straight out of the arm near the implantation site. It is likely that under similar circumstances we could have detected more neural activity, but as explained in the methods section, it is not possible to have the electrode wire come out of the arm's skin when working with monkeys. And one also has to wonder how realistic this situation is for real life use, where the arm is not constantly held stationary. In order to achieve successful neuronal recordings with TIME arrays in the nerves of the arm, the signal needs to be amplified close to the source, which requires implantable amplifiers. Preferably one wants the acquisition system to be completely wireless, as it eliminates the need for a long subcuteanous wire that introduces noise and a percuteanous connector, that introduces infection risk.

Lastly, despite not being able to achieve successful kinematic decodings due to poor recordings, I still want to briefly touch on this subject. With such a high degree of complexity that is involved in controlling the human hand-arm system, with its many muscles and sensors working in synergy, it might seem overzealous to think we intercept, understand, and mimic 
it with neural interfaces linked to prosthetic devices [Castellini and Smagt, 2013]. And while I am also sceptical if we can ever restore the hand's full mobility range, I am of the opinion that this is not necessarily required in order to significantly enhance the quality of life of an amputee patient. [Liu et al., 2014] showed that many of our available hand grip types can be classified under the same group, with only minor differences in aperture. Bullock et al., 2013] even tops this by claiming that $80 \%$ of our daily life activities can be done with 5 -10 different grasps. With this in mind, if we are able to restore a fluent and accurate control of this set of grip types using neural decoders driving a prosthetic hand, a patient would already benefit greatly from it.

\subsection{Somatosensation task}

The somatosensory discrimination task was designed with three goals in mind. First of all, to find out how the animal would react to electrical stimulation, as nerve stimulation with TIME electrodes had, to our knowledge, not been performed on rhesus macaques before. The second was to see how the stimulation parameters would influence the discrimination ability. And lastly to investigate the long term stimulation effects, which will be discussed in the next section.

As this experiment was meant as proof of concept study, I deliberately chose to perform a simple two-alternative forced choice-scheme. While the task cannot determine what kind of percept the electrical stimulation evokes, it does allow the investigation of how the stimulation parameters influence the discrimination ability. It was chosen to do this with simultaneous stimulating of both the median and ulnar nerve came, because Romo and Salinas, 2003 found that sequential stimulation to the same region simply gives rise to a higher/lower classification, regardless of the baseline. This would severely inhibit the ability to investigate how different stimulation parameters affect the sensitivity.

Despite that the electrode longevity prevented the completion of the somatosensory discrimination task training with electrical stimulation, information can still be derived from it. First, 
the animal was able to understand the task very well and was able to discriminate the ulnar and median tactile vibrations with high sensitivity. With respect to the response to the electrical nerve stimulation it can be stated that the animal reacted very well to it: No signs of discomfort were observed in the animal, not even at the maximum stimulation limit where a small muscle twitch occurred. The fact that the animal was able to timely respond to it, does mean it is feeling the evoked sensation. But to explore both simultaneous nerve stimulation discrimination as well as the limits of the ability to discriminate the electrical cues, it is required to have both TIME arrays functioning longer. The lifespan of maximum a couple months is insufficient to train such a complicated task with non-human primates.

The short electrode lifespan also meant that the effects of varying the stimulation patterns could not be investigated, which plays a crucial role in how the electric stimulation is perceived. The stimulation charge determines how many fascicles are recruited, and thus influences the lower sensory thresholds (where nothing is perceived) as well as the upper ones (where potentially pain is evoked). As the sensation of pain had to be avoided, the upper limit was set to the point where movement twitches occurred. Since pain fibres are smaller than the motor efferent or other sensory fibres, they get recruited last Basbaum et al., 2009]. Normal natural fascicle recruitment goes from the smaller to the bigger fascicles, but in general with electrical stimulation this is inversed, as bigger fascicles have lower resistance Raspopovic et al., 2011, Raspopovic et al., 2012. This is not a complete linear correlation though, as factors like endoneurium thickness play a role as well Grinberg et al., 2008. In general, however, it can be said that if one keeps the stimulation charge at or below the minimum movement threshold, pain fibres are not activated. That we did evoke small hand twitches during the training of the electrical stimulation was not a desired effect, but it was necessary to ensure that the electrode channel was actually functional. With amputee patients, who self-explanatory lack hand muscles, this movement twitch would not have been evoked anyway, so potential higher thresholds could be even used in those scenarios.

Another stimulation parameters that effect the perceived sensation is the frequency of the pulse. As discussed before, the PNS is generally rate coded, in which a higher frequency translates to either a stronger muscle contraction or an encoding of a stronger stimulus. However, research 
has shown that there are more factors that encode information. Johansson and Flanagan, 2009] showed that the PNS does not only convey information in rate coding (pulse frequency), but that the relative spike timing also plays a role. [Tan et al., 2015] showed that one can eliminate the feeling of paresthesia (undesired tingly electrical sensation) by simply introducing small variations in the pulse width over time during continuous stimulation. While still unclear how to modulate it best, it can be imagined that other touch sensations than just pressure can be evoked by applying a more complex stimulation pattern. Pruszynski and Johansson, 2014] described how 'edge detection' is already encoded at the PNS level, by summing the information from many sensor types from different locations in the skin. Restoring such fine touch sensations will require complex hardware sensors in the prosthetic skin as well as high stimulation selectivity and more knowledge about the PNS encoding. Apart from somatosensory restoration, nerve stimulation can also be used for treating phantom limb pain, which is an ongoing uncomfortable or painful sensation seemingly coming from the missing limb. There is increasing evidence that an increased sense of embodiment of the limb, strengthened by somatosensory feedback, can reduce these symptoms Ackerley and Kavounoudias, 2015, Tabot et al., 2015, Tan et al., 2015.

This also brings us to the big limitation of the animal model for the exploration of electrical nerve stimulation for the use of somatosensory feedback: the lack of speech or other expressions of experienced percepts. While sensory discrimination is a quantifiable aspect of touch, we are not able to easily identify what kind of percept the stimulation evokes in the animal. That is, whether the animal perceives a natural sensation like pressure or simply paresthesia. As discussed, there are many factors influencing how the electrical sensation is perceived and it remains questionable how dependent the perceived stimulation is on the individual studied. Of course one can think of advanced training schemes with monkeys to investigate which sensory percept is evoked, but in my opinion the animal model is too limited for this aspect of research. With verbal communication between the patient and the experimenter, one can simply ask how the sensation is perceived, which can save many months of animal training time. Regarding biocompatibility and electrode longevity experiments, the animal experiments still have its place, but as soon as we wish to investigate the more complex aspects of somatosensory restoration, 
human experiments, if possible, would be far more promising.

\subsection{Electrode longevity}

Already before the implantation it was known that the implantation time would be limited. In my opinion biocompatibility is still the limiting factor in the field of neurorehabilitation as foreign material in the body still gets rejected at some point. The expected PNS interface lifespans varies strongly on it's invasiveness. With entraneural electrodes (such as the cuff electrode) one can achieve implantation times of a couple of years [Tan et al., 2014], but for intraneural implantations you are limited to a couple of months Navarro et al., 2005. Do note that these expected lifespans are for electrical stimulation, as the ability to record neural activity is lost much sooner due to the formation of scar tissue. In the most recent human experiments the TIME electrodes had to be deplanted after 4 weeks due to ethical/licensing reasons. After this period they reportedly still worked fine, though some channels did fail and the stimulation thresholds had significantly risen [Raspopovic et al., 2012, Rossini et al., 2010]. Rossini et al., 2010 does report that in similar experiments with tf-LIFEs the efficacy already starts dropping 10 days post-surgery.

With this limited implantation time in mind, I planned the experimental time in such a way that the first weeks post-surgery would focus on recording neural data. In this period there is minimal scare tissue formed, so the electrode should perform optimally. After sufficient data was collected, I shifted the focus to the electrical nerve stimulation experiment, as this experiment is still possible to conduct with higher electrode impedances.

Unfortunately we observed complete failure in the first implantation after 2 months and after 2 weeks in the second implantation. Histology showed that for 2 out of the 4 TIME arrays the thin-film had broken due to distal shifting of the cable and adapter. This is likely caused by the muscles tugging and compression of the subcutaneous cable and adapter during movement. Non-human primates also have a much higher mobility than human patients have, especially since the animals had intact limbs, which only increased the amount of stress on the TIME 
arrays. The reason why the other two arrays failed is not clear. It could be that the internal structure of the TIME array was damaged, however, we could not verify this during the deplantation. Neither was the equipment at hand, nor would it be possible to ensure that the damage occurred before or during the perfusion. Most likely, the amount of stress on the array caused it to shift inside the nerve, which could explain the high variation in the stimulation performance of the array during the experiment. Human patients will obviously be more wary of the implanted arm, which could lead to a longer lifespan, but improvements of the electrode design will be necessary to establish long-term peripheral nerve interfacing in non-human primates.

The TIME electrodes are still being further developed and also new type of intrafascicular thin-film electrode arrays are tested. A promising new PNS interface is the SELINE, which three-dimensional self-opening design that hooks itself into the neural tissue Cutrone et al., 2015]. This might solve the issue of the array shifting around in the nerve, but one has to be wary of nerve damage. When there is pressure exerted on the array and the array itself cannot move, the force will be directed into the nerve, which can cause damage. Further testing of this interface is required before it can be applied on (non-human) primates.

\subsection{Outlook}

Durand et al., 2014 addresses three key issues that need to be dealt with in order to drive the neural interface field forward.

1. The ability to access remotely and reliably internal neural signals.

2. A translation strategy taking basic research to the clinic.

3. Fundamental tool development procedures for neural interfacing

The first item, we have already touched upon. It will require the development of an implantable amplifier and a wireless transmission system. It being wireless will also help translation to clinical research. It not only alleviates the need for the patient to be physically tethered to a neural recording system, but it also gives advantages for experimental use in the form of greater 
mobility and less movement artefacts due to cable movement. For BCI-applications, wireless solutions are being developed [Jackson et al., 2006b], but unfortunately they cannot be directly translated for the use of PNS interfaces. First of all, they are often too bulky to implant under the skin in the upper arm. Secondly, they rely on being embedded in the skull, as it is a solid and stable surface. Using a head mounted solution is something one would like to avoid, as it requires a fairly large subcutaneous cable under the skin with all its implications on the signal quality. Miniaturisation of neural processing and decoding equipment to dedicated micro processing chips is the last step, as it will alleviate the need for heavy processing machines.

Lastly, regarding the development of fundamental tools for neural interfacing, it is obvious that the biocompatibility of the neural interfaces needs to increase. The biochemistry field will play an important role in this, as the development of better biocompatible coatings could reduce the rejection rate of the neural interfaces greatly. On the other hand, improvements in robotic systems, like embedding strain gauges and anti-slip mechanisms in the prosthetic hand itself, will also aid in increasing the dexterity, while simultaneously reducing computational demands Carrozza et al., 2002. These, however, both fall out of the scope of this thesis. What does not, and which is often forgotten, is how standardisation of procedures can help the development of tools. For example, regarding the implantation techniques, the wheel is figuratively speaking redeveloped by every surgeon, as there is no standard procedure for it. This can and will cause great disparity in the success rate of the application of neural interfaces. The same can be said regarding the neural processing algorithms and the electrical stimulation pulses. Do note that standardisation comes at the end of the development process. First, further research on among others, the development of efficient neural recording/decoding techniques as well as effective stimulation strategies is required before the scientific community can come to a consensus for a single standard, which will help taking the trial-and-error factor out the equation. 



\section{Chapter 5}

\section{Conclusion}

In this thesis I investigated how well the TIME array can function as a peripheral nervous system interface for both motor control of prosthetic devices and somatosensory feedback. While it is shown that TIME arrays can be implanted in the median and ulnar nerve in the upper arm of a rhesus macaque, without adverse effects. The surgical procedures are not standardised yet and there is no way to ensure that the TIME arrays will be targeting the nerve fascicles.

The recording capability of the TIME was tested with a motor decoding task, in which the animal was trained in a delayed grasping task. Neural activity was detected in some of the recordings, but it was too sparse for meaningful grip type decoding, due to the amount of noise introduced by, among others, the length of the subcutaneous cable.

Electrical stimulation of the nerve for somatosensory feedback was investigated with a twoalternative forced choice task. The animal succeeded in the discrimination of tactile vibration cues simultaneously applied to the median and ulnar region of the hand. Due to premature array failure, the longevity of the TIME arrays was too short to complete the training of the somatosensory discrimination task with electrical stimulation to the nerve. However, the animal did respond well to the electrical stimulation and no signs of discomfort were observed.

In order to use thin-film arrays such as the TIME arrays for peripheral nervous system recordings, it is required to move towards a solution with an implantable amplifier in order to improve the signal-to-noise ratio. A more biocompatible and stable solution is necessary to establish long-term nerve stimulation experiments in non-human primates and ultimately in human patients. 



\section{Appendix A}

\section{Supplemental Material}

\section{A.1 Vibration motor analysis}

To analyse the voltage versus frequency relation of the vibration motors used in the tactile vibration stimulation glove in the somatosensory discrimination task, I was looking for a quick, reliable, and cheap method to test the motors for their durability and the inter-motor rotations per minute (RPM) consistency. The MSc students Laura Jens and Luis Ángel Pardo Sánchez assisted me during the development and testing of the analysis method as well the measuring the motor specifications.

\section{A.1.1 Sound analysis}

The initial idea was to use sound recordings to analyse the vibration frequency. Sound clips of approximately 10 seconds were taken of a vibration motor while it was spinning at a predetermined voltage. Using fast fourier transformation (FFT) in MATLAB the frequency spectrum of the sound clip was obtained and thus the rotational frequency.

We ran into several problems using this method:

- Audibility

Though at high frequencies the motors made sufficient noise to record them well, at low 
voltages the rotational speeds the motor are hardly audible. The low rpm range happened to be exactly in the frequency range in which I intended to use motors, so this formed a challenge. I did not have a sound proof environment at hand, nor sensitive recording equipment. To acquire this would defeat the purpose of having a quick and cheap way to record the vibration frequency.

- Questionable results

While we could extract a frequency using this method, I had doubts about the reliability of the results. The motor specifications listed that the maximum rotations per minute (RPM) would be around 2000, this equals a rotational frequency of around $33 \mathrm{~Hz}$. The results however showed frequencies of up to $200 \mathrm{~Hz}$. This led to speculation whether the method was unreliable or whether also the shape of the head should be taken into account. To illustrate this image performing the same analysis on the sound recording of a 4 bladed helicopter, one would measure a frequency 4 times as high as the actual rotational frequency as you 'hear' 4 blades pass by each rotation. A factor 4 division would bring the $200 \mathrm{~Hz}$ much closer to the $33 \mathrm{~Hz}$ of the specifications, but as can be seen in Chapter 2, the head of the vibration motors are half circles, which would not create a repetitive sound signature during a single rotation.

Taking these two factors into account I decided to look for a new way of recording the actual vibration of the motors using force sensing resistors.

\section{A.1.2 Force sensing resistors}

Force Sensing Resistors (FSR) are robust polymer thick film (PTF) devices that exhibit a decrease in resistance when exposed to an external force to the surface of the sensor. The FSR's chosen for this measurement are the FSR 400 (Interlink Electronics, see figure A.1 and table A.1). In the vibration motor analysis setup, an apparatus (designed and adequately dubbed Contingentia v1.1 by Luis Perdo) presses the vibration motors against these FSRs with constant pressure. The vibration of the motors lead to an oscillating increase and decrease in 
pressure on the sensor, thus it's resistance respectively decreasing and increasing. A resistance hooked in series with the FSR will determines its sensitivity range (higher resistance results in a higher sensitivity). A $10 \mathrm{k} \Omega$ resistor proved to be sufficient to detect the oscillations of the motor. These changes in resistance lead to a change in potential difference around the resistor, which is hooked up to the analog input of a National Instruments (NI) PXI Box. With a special script written in LABVIEW the motors are driven with set of pre-determined voltages and the potential difference around the resistors is recorded simultaneously.

Figure A.1: Force Sensing Resistor (FSR) 400 - Interlink/ $5 \mathrm{~mm}$ Circle x $38 \mathrm{~mm}$. The type of force sensing resistor that was used to analyse the voltage vs frequency relation of the vibration motors used in the somatosensory discrimination task.

Table A.1: Technical specifications - Force Sensing Resistor (FSR) 400]

$\begin{array}{ll}\text { Force Sensitivy Range } & \sim 0.2 \mathrm{~N}-20 \mathrm{~N} \\ \text { Resistance } & 1 \mathrm{M} \Omega-<3 \mathrm{k} \Omega \\ \text { Radius } & 7,62 \mathrm{~mm} \\ \text { Height } & 0,3 \mathrm{~mm} \\ \text { Durability } & 10 \text { Million actuations }(1 \mathrm{~kg}, 4 \mathrm{~Hz})\end{array}$

The following material is required for the motor frequency analysis:

- Holding Apparatus 'Contingentia v1.1'

(holds the vibration motor against the sensor with a constant pressure, see Supplementary Figure A.2

- Trigger box (Supplementary Figure A.3.

- Transistor (2N3704)

- Resistor $(10 \mathrm{k} \Omega)$

- Voltage sources $(2 \mathrm{x})$

- NI PXI Box (to create a specific analog output and receives the analog input)

- PC with working license of LabVIEW and MATLAB 
- LabVIEW program 'Vibration Test 1.2.vi'

- Required variables: Cue duration, pause duration, 9 voltage steps, frequency, number of recorded samples.

- MATLAB-scripts:

- 'Analog Input Analysis.m' for the analysis of a single motor

- 'MotorComparison.m' for the comparison of two motors,

- required function: 'analogInputAnalysis.m'
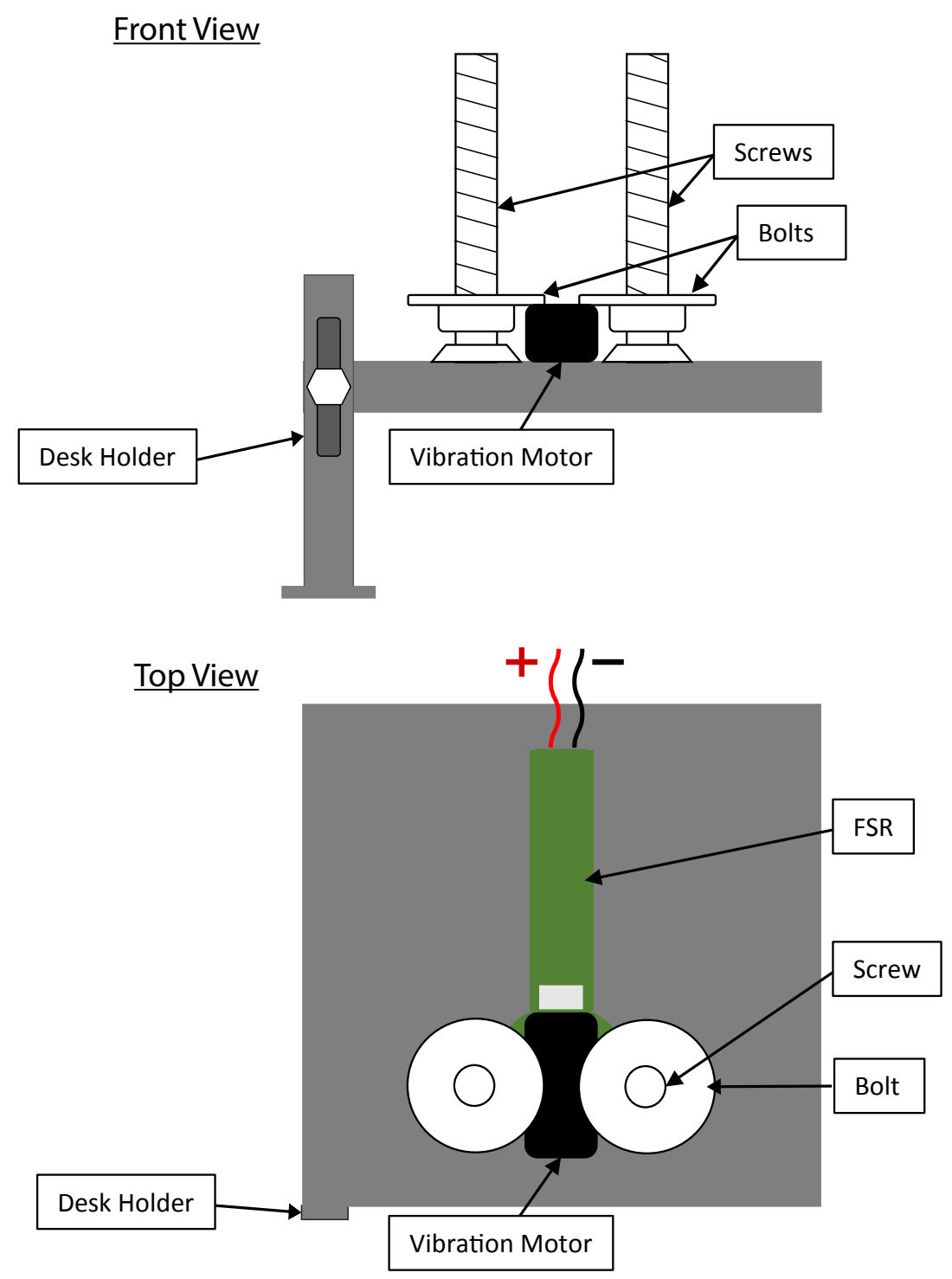

Figure A.2: Motor holding apparatus 'Contingentia v1.1' - Schematic drawing of the apparatus used to keep the vibration motors pressed to the FSR with constant pressure. The motor is placed on the FSR and the bolts are screwed down till the motor is pressed sturdily against the FSR without shifting during vibration. 


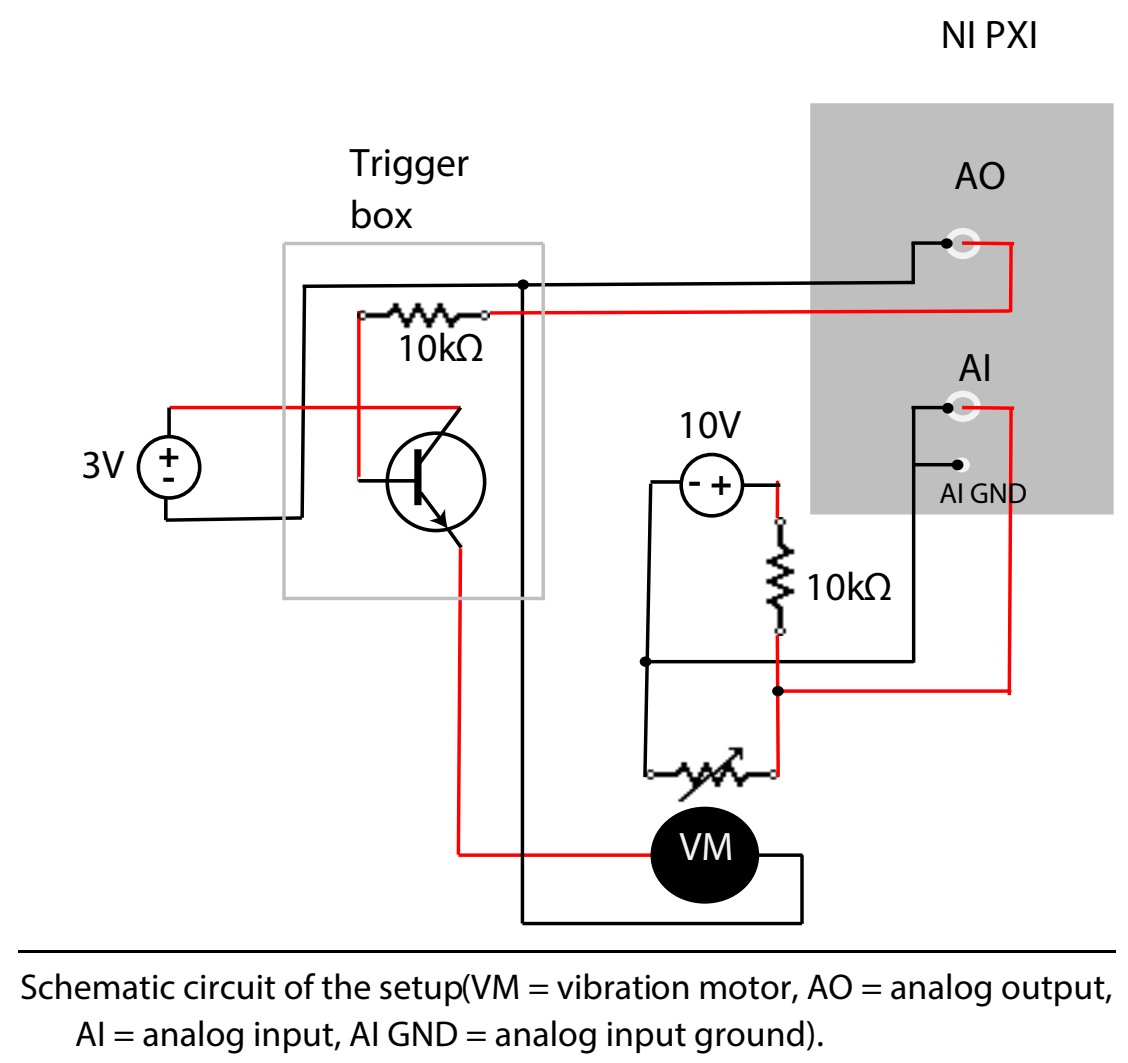

Figure A.3: Wiring schematic of vibration motor analysis setup - Shows the complete electric wiring schematic for the recording of the voltage oscillations that determine the rotational speed of the vibration motors.

\section{A.1.3 Motor Results}

- Motor durability

Vibration motors of this size are brushed motors and these will wear over time. To see how long a motor can be used without changing its frequency at defined voltages, we span a motor continuously for 3 hours every day at 5V (LabVIEW I/O). After each hour, the vibration frequency was recorded for voltages of $3 \mathrm{~V}, 5 \mathrm{~V}$ and $7 \mathrm{~V}$. This test was performed over a week, spanning in total 20 hours. It was found that after 17 hours the motors would not spin at $3 \mathrm{~V}$ anymore and the motors were declared defective at this point. Because the motors are able to withstand such heavy abuse, they were deemed durable enough for the somatosensory discrimination task in which they would only spin for a fraction of second during each trial. 
- Inter-Motor consistency

During the experiments two motors are used at the same time, which makes it important that the two motors don't differ too much in respect to their vibration frequency. The frequencies of 12 different motors were recorded twice at different voltages to compare them between each other (see Figure A.4). A fairly linear relationship is observed between the 2.5-6V range after which the curve slopes down.

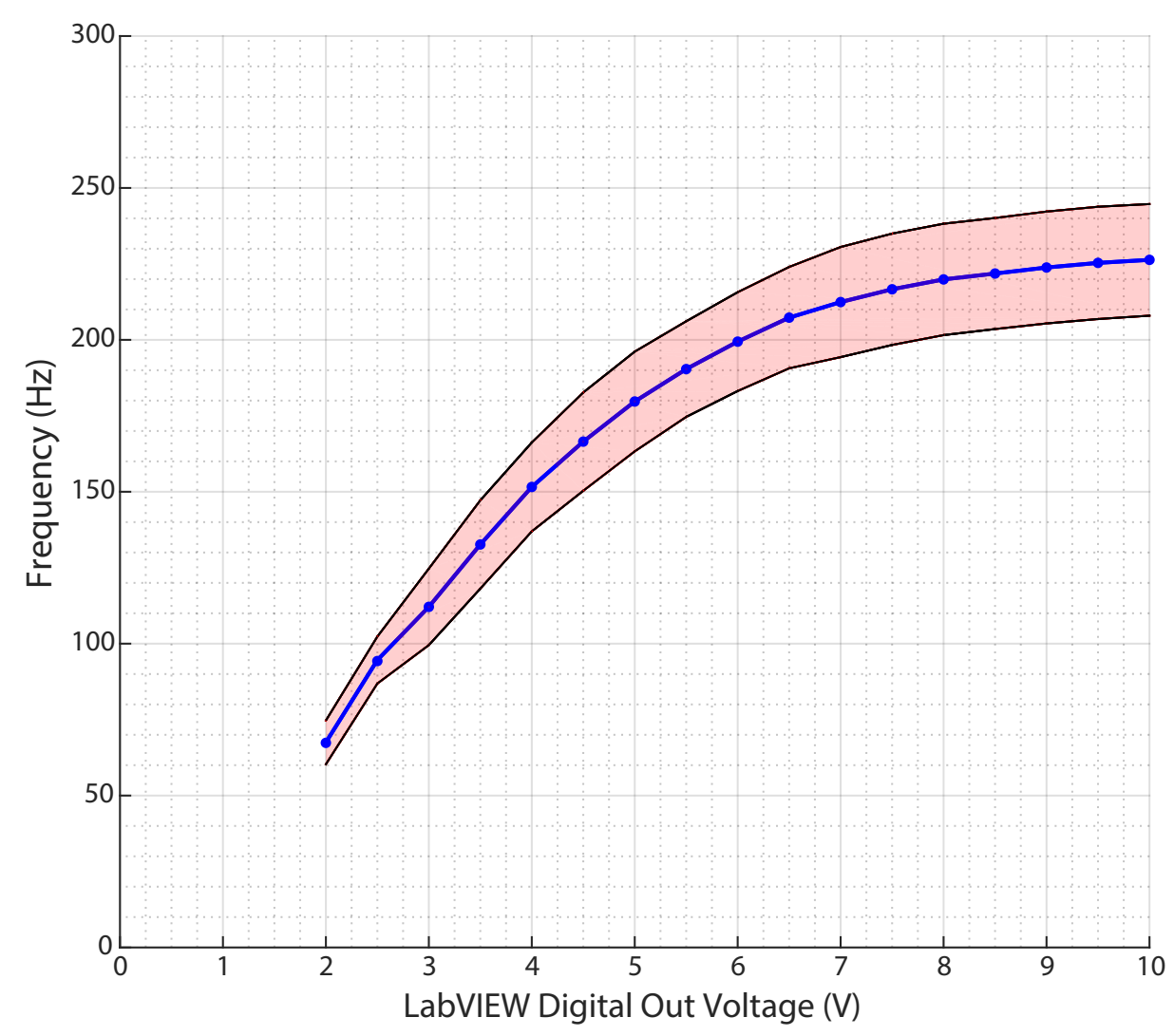

Figure A.4: Motor frequency consistency - The voltage vs frequency relationship measured for 12 vibration motors shows a fairly linear relationship in the $2.5-6 \mathrm{~V}$ range after which it slopes off. 


\section{A.2 Somatosensory discrimination task}

\section{A.2.1 Performance Chart - Vibration Only}

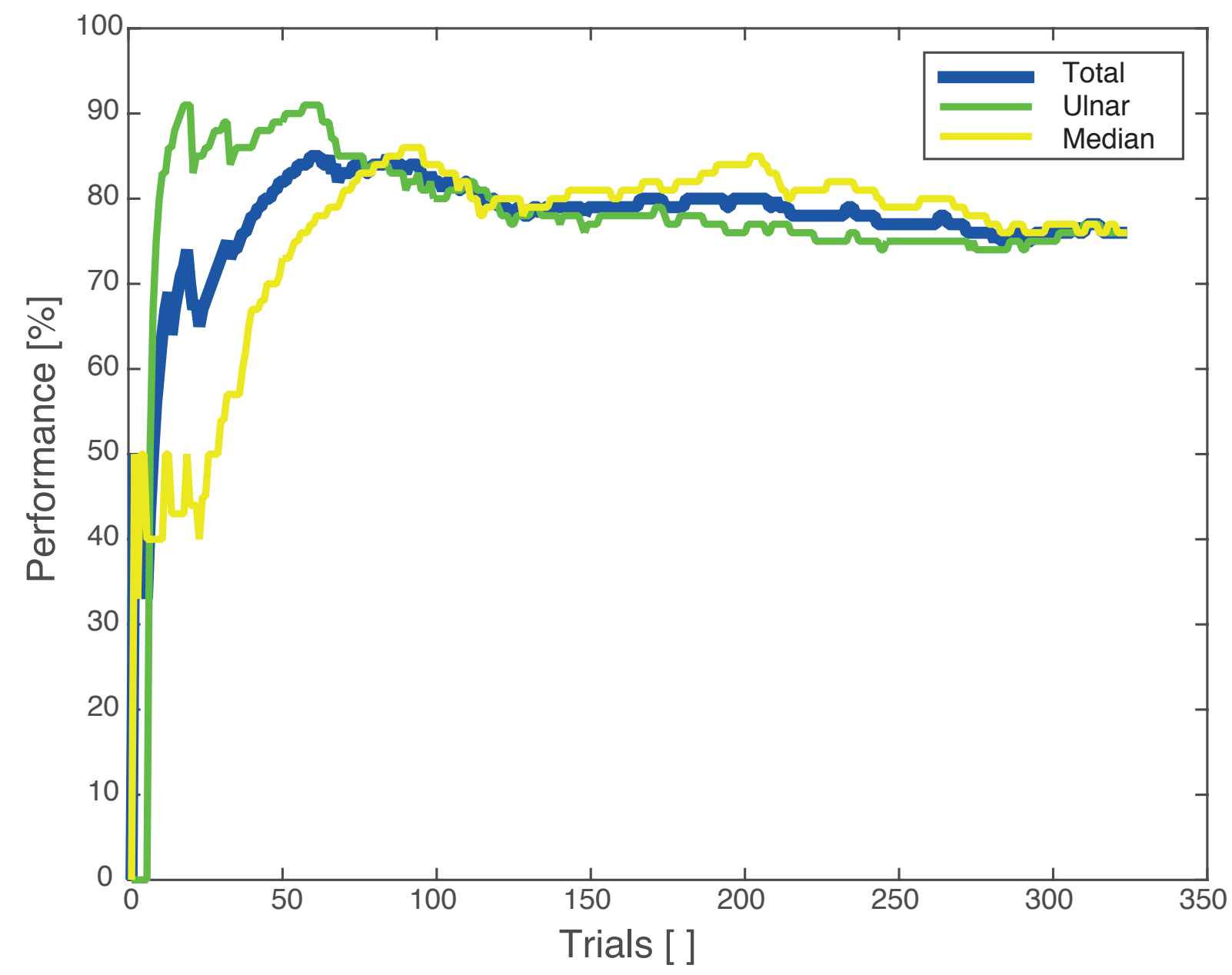

Figure A.5: Performance Chart Somatosensory discrimination task - Graph of the performance over time for the somato-sensory discrimination task with vibration only stimulation within the range of 100-200 Hz simultaneous cues. Total performance for this specific recording shortly peaked around $85 \%$ and then stabilises around the $80 \%$ for both ulnar as median stimulation types. 



\section{Appendix B}

\section{Side Project - Releasable Collar}

\section{B.1 Abstract}

For behavioural monitoring of animals in the wild, amongst others GPS and vocal recordings are well known scientific methods. These however require capturing of the animal to put on the collar and another time to remove it after data acquisition. While the former is quite inevitable, the latter can be prevented by using remotely releasable collars. Currently there is no such collar that is designed for small animals $(<10 \mathrm{~kg})$ that is also reusable. In this section the design for a collar for red-fronted lemurs is presented, which is remotely releasable and weighs less than 50g. It is operated with an Android app over a Bluetooth connection, has a standby time of weeks and has a material costs of less than $€ 50,-$. Custom purpose software for both the Arduino controlling the collar and the app operating the Arduino was also developed.

The Remote Releasable Collar project is done in collaboration with Louise Peckre, from the Behavioral Ecology \& Sociobiology Lab at the Deutsches Primatenzentrum GmbH. Matthias Dörge, the technical assistant of the Neurobiology Lab of the DPZ, assisted in building the prototypes. 


\section{B.2 Introduction}

Many behavioural studies on rely on the monitoring of animals in the wild. Preferably this is done remotely as it ensures the animal is behaving naturally without the influence of humans. Depending on the type of study, they often require the recording of vocal callings or tracking of GPS position. The data acquisition hardware is then often mounted on a collar. Most existing collars require to capture the animal twice, once to put on the collar and again to take it off. This is not only tedious for the researcher, but more importantly, it evokes evokes a lot of stress for the animal.

There are collar designs that allow either remote, or time triggered release modes, but they are mostly designed for larger animals and have explosive release mechanisms Marshall, 2007. Marshall et al., 2005. This makes them heavy and bulky, and thus not suitable for smaller mammals. As a further disadvantage, those explosive release mechanisms are not re-usable and require replacement of (a part of) the locking mechanism in order to use the collar again.

Here I present a collar that is remotely releasable over Bluetooth, light weight $(<50 \mathrm{~g})$, re-usable, cheap to manufacture (<€50,-), with excellent battery life (over a year standby) that has been tested for its use of vocal recordings on red fronted lemurs.

\section{B.3 Methods}

\section{B.3.1 Specifications}

An important consideration in the design of the collar was the size and weight limits of the collar. The red fronted lemur, the animal the collar is designed for, is a small primate. With an average weight of $\sim 2 \mathrm{~kg}$, the lemur would be hindered in its movement if the collar is too bulky or heavy. In coordination with Behavioral Ecology \& Sociobiology Lab at the Deutsches Primatenzentrum GmbH, it was found that GPS collars, currently in use for this species, have a weight of $\sim 50 \mathrm{~g}$, so it was decided to set this as the maximum weight of the new collar design. 


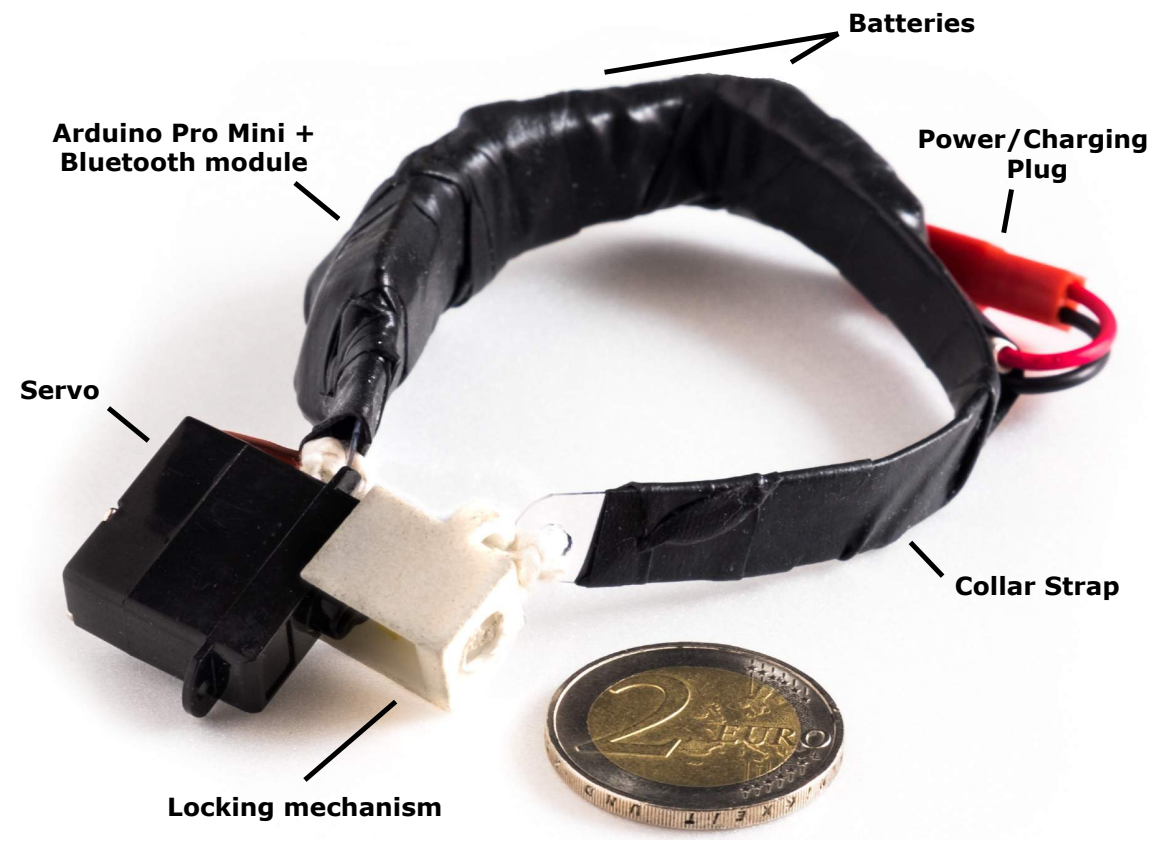

Figure B.1: Releasable Collar - Shows the collar in its locked state with its internal components labelled. All the electronics are wrapped in self-fusing silicone rubber tape to provide basic protection from the elements. Only the power/charging plug is left bare to allow charging and powering on the device (note it is recommended to seal it when using the collar in the field).

Because the collar is remotely releasable it will contain electronics and this imposed weight limit, and thus indirectly the size limit, has the biggest effect on the battery life of the collar. Typical vocal recordings last a couple of days till a week, so allowing a similar operation time of the collar was our aim. The power consumption varies drastically between the active and sleep mode.

The last specification it had to fulfil is the ability to control it remotely. A Bluetooth 4.0 connection was chosen as the communication interface for its diversity and range. While other solutions like UHF (ultra high frequency) solutions like $433 \mathrm{MHz}$ transmitters were considered for their long range, these analog interfaces have the downside that the integrity of the signal cannot be assured. With a digital connection like Bluetooth one can assure that the command, that is sent to the device, will arrive there as long as the device is connected. The actual range that can be achieved with the used Bluetooth receiver depends on both the receiver and transmitter strength, and whether there is any obstruction between them. Tested in an open space we achieved a range of 10-15 meters. When using the collar in a dense forest the range will be reduced by half. As the red-fronted lemur colony in this study is easily approachable by 
the behavioural researchers, this range suffices even if the animals have are climbed up a tree.

A big strength of this collar design is, that with it's low weight one can mount a wide range of data acquisition devices to it. While it was tested with a vocal recorder, mounting, e.g. a stand-alone GPS module is also possible. If longer battery range is desired, there is space to mount an extra battery to it. Finally, the software is flexible to handle custom sleep times with minute accuracy, so precise release timing is possible.

\section{B.4 Hardware}

The presented collar contains the following parts listed in Table B.1. Including the wiring and the wrapping, the collar weighs $25 \mathrm{~g}$, which is ample below the upper weight limit. The total costs of these parts is below $€ 50$,- (labour costs not included) and they are all readily available in hardware stores, making this design cheap and fairly easy to manufacture. The electronic schematic can be found in Figure B.2.

The collar's processing unit is the $8 \mathrm{MHz}$ version of the Arduino Pro Mini. At time of writing it is one of the smallest form factor and readily available single-board microcontrollers on the market. The $8 \mathrm{MHz}$ version is chosen for its positive effect on the battery life. First, its voltage requirements are lower (3.3 instead of $5 \mathrm{~V})$. Second, it allows the use of single cell LiPo batteries. The $16 \mathrm{MHz}$ Arduino Pro Mini would have required two LiPo cells in series to run, which would increase the bulk on the collar. It is also worth noting that for this application the processing speed of $8 \mathrm{MHz}$ processing speed is fast enough.

\section{B.4.1 Locking Mechanism}

The locking mechanism of the collar consists of a servo with a screw mounted on the axis and a 3D-printed casing made from polylactic acid (PLA). The casing can slide around the servo and by activating the servo it can screw itself tight in. When in the locked position, the reverse rotation direction will cause the casing to detach from the servo. An overview of the locking 
Table B.1: Part List - Releasable Collar

\begin{tabular}{l|c|c} 
Part & Quantity & Weight \\
\hline Arduino Pro Mini & 1 & $2 \mathrm{~g}$ \\
Lipo battery, 1S 110 mAh & 2 & $4 \mathrm{~g}$ \\
HC-05 Bluetooth Module & 1 & $1 \mathrm{~g}$ \\
Turnigy Micro servo & 1 & $5 \mathrm{~g}$ \\
Resistor $2.2 \mathrm{k} \Omega$ & 2 & $\sim$ \\
Resistor $1 \mathrm{k} \Omega$ & 1 & $\sim$ \\
Resistor $275 \Omega$ & 1 & $\sim$ \\
Transistors $(2 \mathrm{~N} 3704)$ & 2 & $\sim$ \\
3D Printed lock + screw & 1 & $2 \mathrm{~g}$ \\
Plugs (male + female) & 1 & $1 \mathrm{~g}$ \\
Wiring and wrapping & $\mathrm{n} / \mathrm{a}$ & $7 \mathrm{~g}$ \\
\hline Total & & $22 \mathrm{~g}$
\end{tabular}

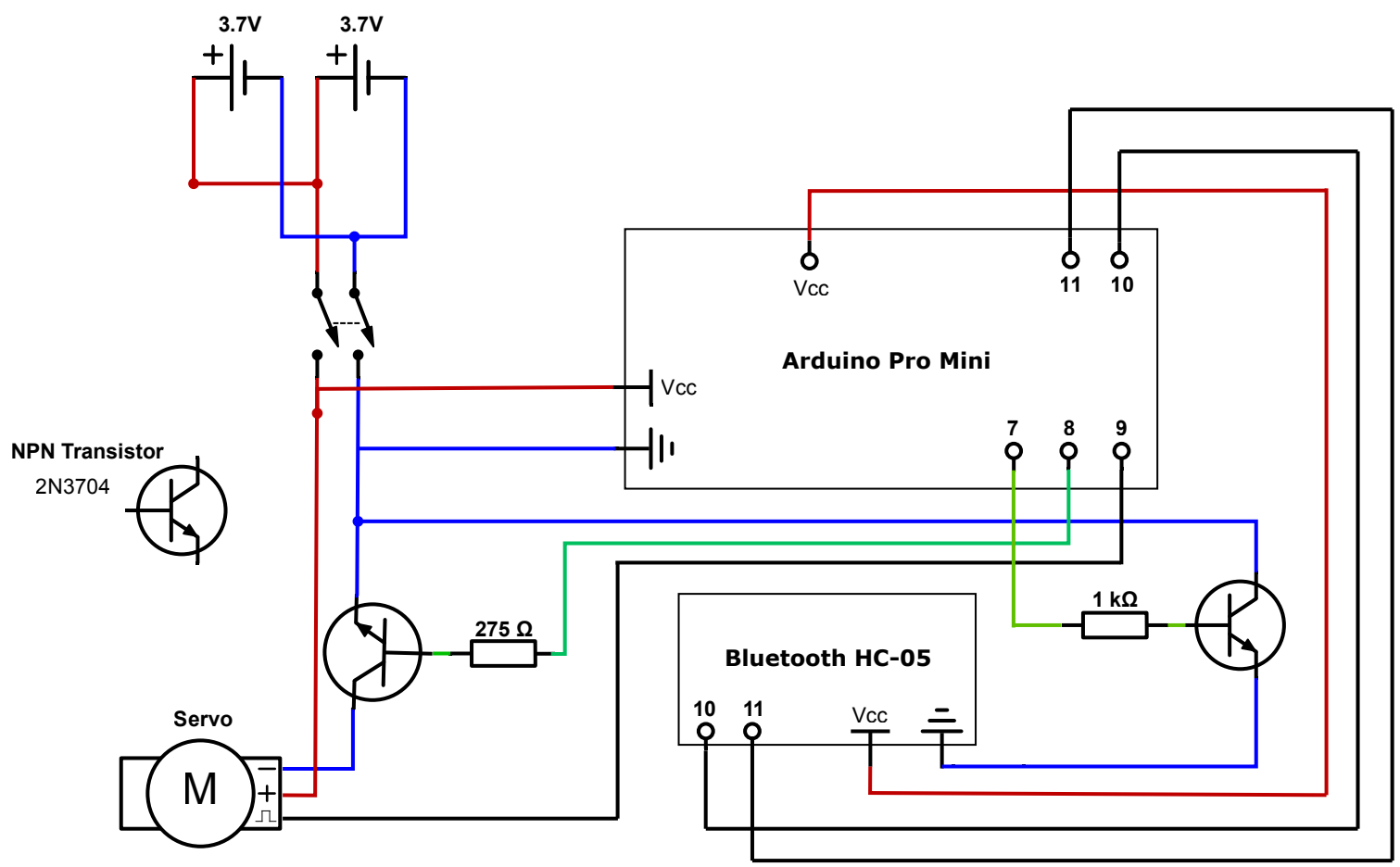

Figure B.2: Wiring Schematic - Shows the electronic wiring schematic of the releasable collar with the Arduino Pro Mini and HC-05 Bluetooth module pinout. Blue wires indicate GND, red wires positive leads and the green wires are triggers (off, $0 \mathrm{~V}$; on, 3.3V. LiPo Batteries are each 1cell (3.7V) and are placed in parallel.

mechanism can be seen in Figure B.3. The servo is modified so it can rotate indefinitely in a single direction. This is achieved by removing the physical end point barriers and replacing the potentiometer inside by two $2.2 \mathrm{k} \Omega$ resistors. A servo is chosen as the driving mechanism for the lock, as it is able to exert a high torque, which prevents the locking mechanism from 
jamming when it is under pressure.

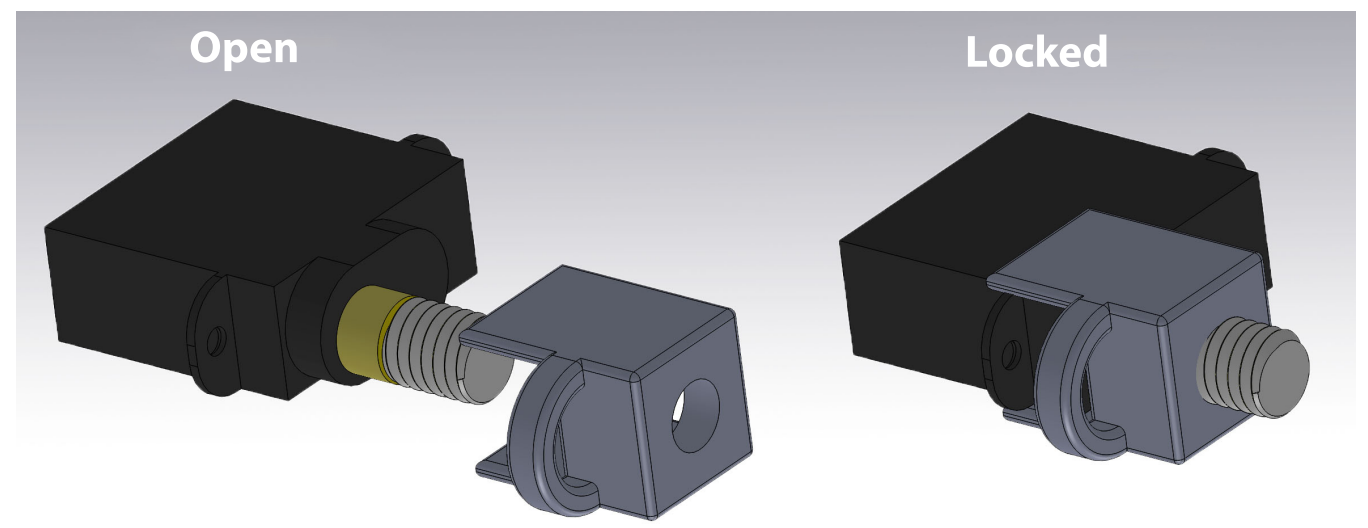

Figure B.3: Locking Mechanism - By activating the servo clockwise or counter clockwise, it will screw itself respectively in and out of the 3D-printed casing. The collar band and the rest of the electronics are attached to the rings at both ends.
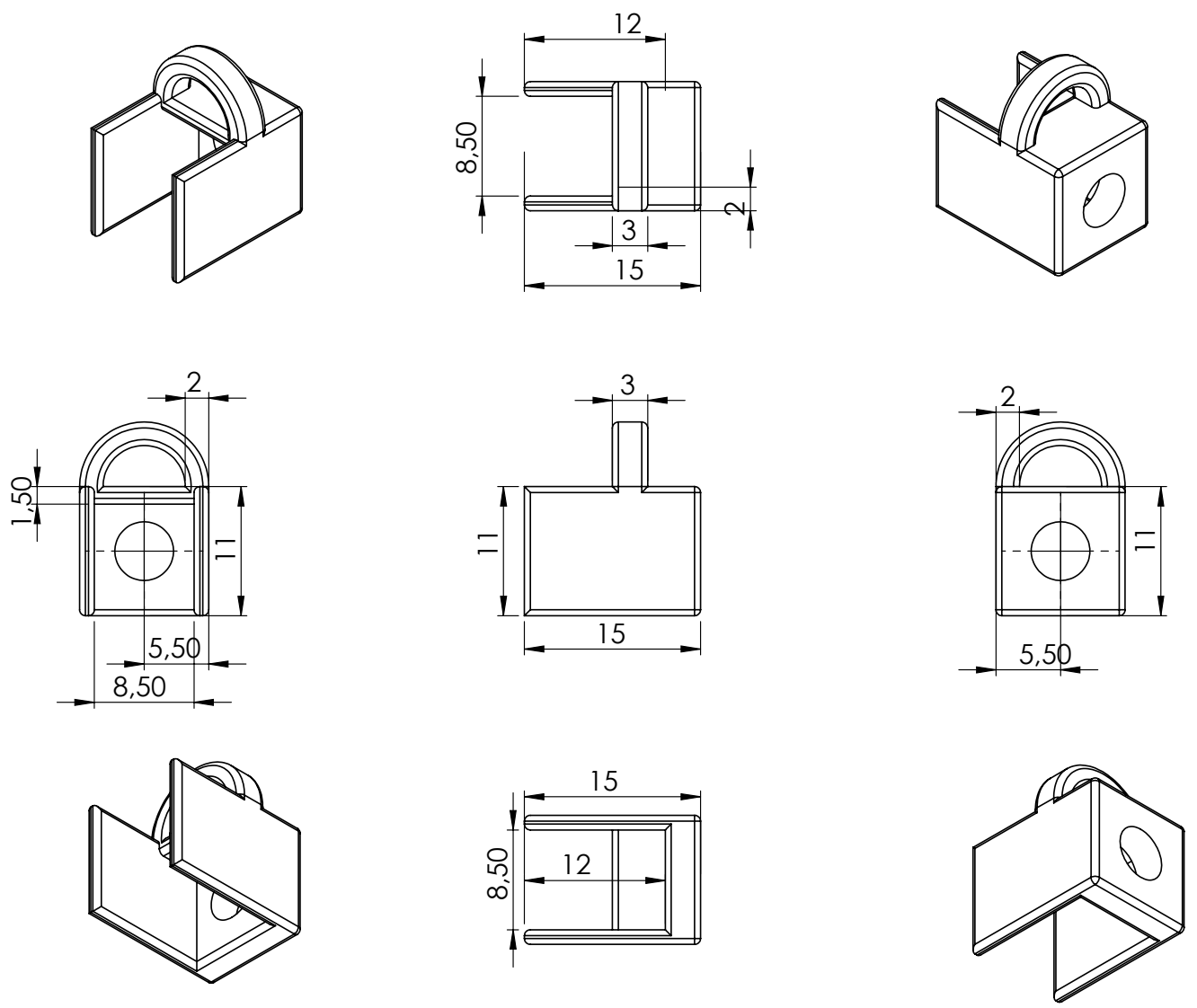

Figure B.4: Schematic Drawing Lock Mechanism - Shows the dimensions of the 3D printed cover for the lock mechanism, note the drawing does not display that the hole is threaded (size M6, ISO metric screw thread). 


\section{B.4.2 Software}

The Arduino runs on a custom made script and is operated by an Android app. Upon initialisation of the Arduino, it opens a Bluetooth connection and initialise the required ports for communication between the app and the device. After the initialisation phase it enters the main loop, where it periodically (every 3ms) checks if there is (serial) data send over the Bluetooth connection. These can be 1 out of 3 commands: open Collar, close collar and initiate sleep mode for a given period. The open and close command drive the servo motor to open or close the collar, respectively. The sleep mode disables the Bluetooth connection and puts the Arduino into a lower power state. This mode is crucial for battery saving and without it the battery would be drained in a matter of hours. More on the power consumption in a later section.

The Android app is a custom made graphical user interface (GUI) developed in MIT App Inventor that allows pairing with Bluetooth enabled devices and is used to open an close the collar. To put the collar into it's sleep state one has to set a date and time at which the device should wake up. An overview of the app can be seen in Figure B.5.

Both scripts have the needed fail safes build in to ensure safe use for both the animal as well as to prevent equipment damage.

\section{B.4.3 Power consumption}

The Arduino in its active state, with the Bluetooth module and servo draws between 12 and $100 \mathrm{~mA}$, when it is idle or driving the servo motor, respectively. Even in the idle state it would drain the two $110 \mathrm{mAh} \mathrm{LiPo} \mathrm{batteries} \mathrm{in} \mathrm{series} \mathrm{within} \mathrm{a} \mathrm{day,} \mathrm{which} \mathrm{would} \mathrm{be} \mathrm{too} \mathrm{short} \mathrm{for}$ practical use. Increasing the lipo battery count is not a optional choice as it would increase the bulk too much. Instead of increasing the battery capacity, decreasing the power consumption is a more viable option.

When the 3.3V 8MHz Arduino Pro Minis microcontroller unit (MCU), the ATmega328P, is 


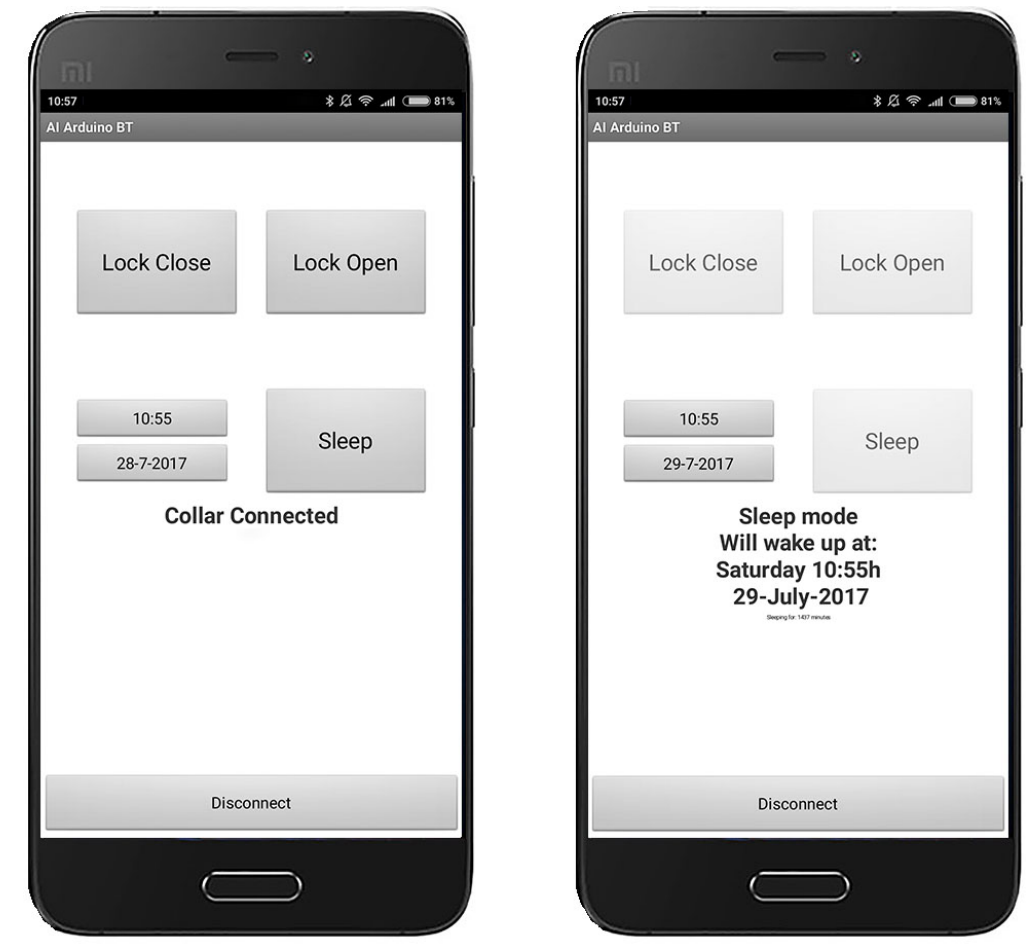

Figure B.5: Android GUI - Show screenshots of the Android GUI that runs on Android which connects to the Arduino mounted on the collar and allows it to be opend and closed remotely (left) and put it in Powered Down Sleep mode for a custom set period (right).

in its Active Mode (ACT) it continuously performs 8 million instructions per second. Aside from that there are several On-Board peripherals that draw power, i.e. the Analog to Digital Converter (ADC), Serial Peripheral Interface (SPI), Timer 0, 1, 2, Two Wire Interface (I2C), USART Watchdog Timer (WDT) and the Brown-Out Detection (BOD). By activating certain sleep modes, the ATmega328p MCU can turn off some of these peripherals [Unknown, 2017].

The Low-Power Library from Rocketscream can disable the ACD and BOD for a pre-specified time. During the 'Powered-Down Sleep' (PDS) mode, these chip functions are disabled until the next interrupt. With the sleep \{forever\} argument, the WDT can also be disabled, but the Arduino will not wake until an interrupt is manually triggered. The latter is not desired for this application, but as Table B.2 shows the PDS mode can decrease the power consumption by a factor 2 while the BT and servo are disabled.

To further decrease the power consumption a modification to the Arduino Pro Mini's board is required. The Arduino Pro Mini has a status and power LED on the board, of which the latter cannot be switched off and the former is still active during sleep mode. Disabling these 
conserves up to $6 \mathrm{~mA}$ in current draw. This requires a hardware modification of either removing or physically disconnecting the LEDs from the circuit board.

These modifications allow the device to stay in sleep mode for roughly a month, but one can extend this time even further by removing the voltage regulator from the board as well. While this current draw reduction of $\sim 0.27 \mathrm{~mA}$ doesn't seem significant compared to the $12 \mathrm{~mA}$ it draws in it's active state, it is a factor 40 decrease when the device is in PDS mode with the LED's disabled. With a battery capacity of $220 \mathrm{mAh}$ the Arduino can run for well over a year.

Table B.2: Power consumption chart - ACT = Active Mode $;$ PDS = Powered-Down Sleep

\begin{tabular}{|c|c|c|c|}
\hline ATmega328P Pro Mini 8 MHz & & & \\
\hline States & Unmodified & NO LEDs & No Voltage regulator \\
\hline ACT, BT disconnected, servo Idle & $62 \mathrm{~mA}$ & $57 \mathrm{~mA}$ & $57 \mathrm{~mA}$ \\
ACT, BT connected, servo spinning & $90-100 \mathrm{~mA}$ & $90-100 \mathrm{~mA}$ & $90-100 \mathrm{~mA}$ \\
ACT, BT disabled, Servo Disabled & $\sim 12 \mathrm{~mA}$ & $\sim 6 \mathrm{~mA}$ & $\sim 6 \mathrm{~mA}$ \\
PDS, BT disabled, Servo Disabled & $\sim 6 \mathrm{~mA}$ & $\sim 0.28 \mathrm{~mA}$ & $\sim 0.007 \mathrm{~mA}$ \\
\hline
\end{tabular}

Note that while the battery drain during sleep mode is minimal, the device consumes significantly (factor 1000!) more energy when it wakes again. So while a few mAh would already provide a long standby time, the battery would rapidly drain when waking and thus would limit the time during which the experimenter can reconnect with the collar to open it. Therefor a middle ground is struck and chosen for a battery capacity of $220 \mathrm{mAh}$, which allows both hours of standby time and many months of sleep time.

\section{B.5 Discussion \& Conclusion}

The releasable collar presented here shows it is possible to construct a remotely releasable data acquisition collar for small mammals, that is cheap to produce and is reusable. The final design of the collar has only been tested under artificial circumstances, however, we do not foresee any problems with the field tests on red-fronted lemurs. An early prototype, with a different locking mechanism has been tested by Louise Peckre at the DPZ field station on Madagascar. Unfortunately that collar could not be retrieved, because the early locking mechanism did not 
forcefully open the collar completely, therefore the collar did not immediately fell off the lemur's neck and was lost in the bushes when it fell off when the animal jumped away. New test at the Affenwald Strauberg will determine if the new design which actively opens, will fall off the neck immediately.

While I do not plan to develop the collar further, there are still small improvements that one could consider implementing.

First, while the silicone wrapping protects the device from water splashes, it is far from waterproof. Especially one needs to be wary that water does not come near the battery connector. Fortunately, the red-fronted lemurs are not keen on swimming and the collar will be used in the dry seasons in the forest in Madagascar. The combination of these two points makes it unlikely that the collar will get in contact with water and therefore waterproofing was of a low priority.

Because the red-fronted lemurs have relatively weak hand coordination and poor hand strength, the device should be strong enough to withstand their potential meddling with the collar. However for bigger, stronger or more aggressive primates, such as rhesus macaques, the design is unfortunately not strong enough. For these species, the weight is less of a limiting factor, therefore one could replace the plastic parts for metal ones, which will increase the strength. With light alloys as aluminium the weight increase will be limited, but the durability will be increased with the added benefit that it is prone to rusting.

When using this collar design on different species one should also consider it's operating range. It is crucial to take into account how close you can get to the animal for the collar retrieval procedure. Red-fronted lemur colonies are not particularly shy and can be approached within a 10 meters. However when it is not possible to get within $\sim 10 \mathrm{~m}$ of the target animal, one should consider a different communication interface, such as $433 \mathrm{MHz}$ UHF communication.

Another improvement that will aid the retrieval of the collar is a low-voltage sensor that can detect when the battery voltage is too low and will subsequently wake the device and open it. This in combination with a piezo buzzer, that will periodically give a loud auditory signal, will help in the retrieval of the collar when it falls in between dense vegetation. These additions 
will not add much more costs nor bulk on the device. 



\section{Bibliography}

[Ackerley and Kavounoudias, 2015] Ackerley, R. and Kavounoudias, A. (2015). The role of tactile afference in shaping motor behaviour and implications for prosthetic innovation. Neuropsychologia.

[Badia et al., 2011a] Badia, J., Boretius, T., A, P., Udina, E., Stieglitz, T., and Navarro, X. (2011a). Biocompatibility of chronically implanted transverse intrafascicular multichannel electrode (TIME) in the rat sciatic nerve. Ieee T Bio-med Eng, 58(8):2324-2332.

[Badia et al., 2011b] Badia, J., Boretius, T., Andreu, D., Christine, A., Stieglitz, T., and Navarro, X. (2011b). Comparative analysis of transverse intrafascicular multichannel, longitudinal intrafascicular and multipolar cuff electrodes for the selective stimulation of nerve fascicles. J Neural Eng, 8(3):036023.

[Barrese et al., 2013] Barrese, J. C., Rao, N., Paroo, K., Triebwasser, C., Carlos, V., Franquemont, L., and Donoghue, J. P. (2013). Failure mode analysis of silicon-based intracortical microelectrode arrays in non-human primates. J Neural Eng, 10(6):066014.

[Basbaum et al., 2009] Basbaum, A. I., Bautista, D. M., Scherrer, G., and Julius, D. (2009). Cellular and molecular mechanisms of pain. Cell, 139(2):267-84.

[Behan et al., 2017] Behan, J., Higgins, S., and Wysong, A. (2017). Safety of cochlear implants in electrosurgery: A systematic review of the literature. Dermatol Surg, 43(6):775.

[Bensmaia and Miller, 2014] Bensmaia, S. and Miller, L. (2014). Restoring sensorimotor function through intracortical interfaces: progress and looming challenges. Nature Reviews Neuroscience. 
[Boretius, 2012] Boretius, T. (2012). TIME-3H - electrode user manual v4.

[Boretius et al., 2010] Boretius, T., Badia, J., Aran, P., Schuettler, M., Navarro, X., Yoshida, K., and Stieglitz, T. (2010). A transverse intrafascicular multichannel electrode (TIME) to interface with the peripheral nerve. Biosens Bioelectron, 26(1):62-69.

[Borra et al., 2008] Borra, E., Belmalih, A., Calzavara, R., Gerbella, M., Murata, A., Rozzi, S., and Luppino, G. (2008). Cortical connections of the macaque anterior intraparietal (AIP) area. Cereb Cortex, 18(5):1094-1111.

[Borra et al., 2010] Borra, E., Belmalih, A., Gerbella, M., Rozzi, S., and Luppino, G. (2010). Projections of the hand field of the macaque ventral premotor area f5 to the brainstem and spinal cord. J Comp Neurol, 518(13):2570-2591.

[Bullock et al., 2013] Bullock, I., Zheng, J., Rosa, S., Guertler, C., and Dollar, A. (2013). Grasp frequency and usage in daily household and machine shop tasks. Ieee T Haptics, 6(3):296-308.

[Carpaneto et al., 2012] Carpaneto, J., Raos, V., Umiltà, M., Fogassi, L., Murata, A., Gallese, V., and Micera, S. (2012). Continuous decoding of grasping tasks for a prospective implantable cortical neuroprosthesis. J Neuroeng Rehabil, 9(1):1-6.

[Carpaneto et al., 2011] Carpaneto, J., Umiltà, M., Fogassi, L., Murata, A., Gallese, V., Micera, S., and Raos, V. (2011). Decoding the activity of grasping neurons recorded from the ventral premotor area f5 of the macaque monkey. Neuroscience, 188:80-94.

[Carrozza et al., 2006] Carrozza, M., Cappiello, G., Micera, S., Edin, B., Beccai, L., and Cipriani, C. (2006). Design of a cybernetic hand for perception and action. Biol Cybern, 95(6):629644.

[Carrozza et al., 2002] Carrozza, M., Massa, B., and Micera, S. (2002). The development of a novel prosthetic hand-ongoing research and preliminary results. IEEE/Asme.

[Castellini and Smagt, 2013] Castellini, C. and Smagt, P. (2013). Evidence of muscle synergies during human grasping. Biol Cybern, 107(2):233-245. 
[Choi et al., 2017] Choi, I., Rhiu, I., Lee, Y., Yun, M., and Nam, C. (2017). A systematic review of hybrid brain-computer interfaces: Taxonomy and usability perspectives. PloS one.

[Christel and Billard, 2002] Christel, M. and Billard, A. (2002). Comparison between macaques and humans kinematics of prehension: the role of morphological differences and control mechanisms. Behav Brain Res, 131(1-2):169-184.

[Clark et al., 2011] Clark, G., Ledbetter, N., Warren, D., and Harrison, R. (2011). Recording sensory and motor information from peripheral nerves with utah slanted electrode arrays. Conf Proc Annu Int Conf Ieee Eng Medicine Biology Soc Ieee Eng Medicine Biology Soc Annu Conf, 2011:4641-4644.

[Clark et al., 2014] Clark, G., Wendelken, S., and Page, D. (2014). Using multiple high-count electrode arrays in human median and ulnar nerves to restore sensorimotor function after previous transradial amputation of the hand. in Medicine and .

[Collinger et al., 2013] Collinger, J., Wodlinger, B., Downey, J., Wang, W., Elizabeth, T., Weber, D., Angus, M., Velliste, M., Boninger, M., and Schwartz, A. (2013). High-performance neuroprosthetic control by an individual with tetraplegia. Lancet, 381(9866):557-564.

[Council, 2003] Council, N. (2003). Guidelines for the care and use of mammals in neuroscience and behavioral research.

[Cutrone et al., 2015] Cutrone, A., Valle, J., Santos, D., Badia, J., Filippeschi, C., Micera, S., Navarro, X., and Bossi, S. (2015). A three-dimensional self-opening intraneural peripheral interface (SELINE). J Neural Eng, 12(1):016016.

[Danilov and Tyler, 2005] Danilov, Y. and Tyler, M. (2005). Brainport: an alternative input to the brain. Journal of integrative neuroscience.

[Dann et al., 2016] Dann, B., Michaels, J. A., Schaffelhofer, S., and Scherberger, H. (2016). Uniting functional network topology and oscillations in the fronto-parietal single unit network of behaving primates. Elife, 5 . 
[Dario et al., 1998] Dario, P., Garzella, P., Toro, M., and Micera, S. (1998). Neural interfaces for regenerated nerve stimulation and recording. IEEE Transactions .

[Delgado-Martínez et al., 2016] Delgado-Martínez, I., Badia, J., Pascual-Font, A., RodríguezBaeza, A., and Navarro, X. (2016). Fascicular topography of the human median nerve for neuroprosthetic surgery. Front Neurosci, 10:286.

[Dhillon and Horch, 2005] Dhillon, G. and Horch, K. (2005). Direct neural sensory feedback and control of a prosthetic arm. Ieee T Neur Sys Reh, 13(4):468-472.

[Dhillon et al., 2005] Dhillon, G., Krüger, T., Sandhu, J., and Horch, K. (2005). Effects of Short-Term training on sensory and motor function in severed nerves of Long-Term human amputees. J Neurophysiol, 93(5):2625-2633.

[Durand et al., 2014] Durand, D., Ghovanloo, M., and Krames, E. (2014). Time to address the problems at the neural interface. J Neural Eng, 11(2):020201.

[Durand et al., 2005] Durand, D., Grill, W., and Kirsch, R. (2005). Electrical stimulation of the neuromuscular system. Neural engineering.

[Farrell and Weir, 2005] Farrell, T. and Weir, R. (2005). Pilot comparison of surface vs. implanted EMG for multifunctional prosthesis control. Rehabilitation Robotics.

[Farrell and Weir, 2008] Farrell, T. and Weir, R. (2008). A comparison of the effects of electrode implantation and targeting on pattern classification accuracy for prosthesis control. Ieee T Bio-med Eng, 55(9):2198-2211.

[Ganguly and Carmena, 2009] Ganguly, K. and Carmena, J. M. (2009). Emergence of a stable cortical map for neuroprosthetic control. Plos Biol, 7(7):e1000153.

[Gijsberts et al., 2014] Gijsberts, A., Bohra, R., González, D., Werner, A., Nowak, M., Caputo, B., Roa, M., and Castellini, C. (2014). Stable myoelectric control of a hand prosthesis using non-linear incremental learning. Frontiers Neurorobotics, 8:8. 
[Grinberg et al., 2008] Grinberg, Y., Schiefer, M. A., Tyler, D. J., and Gustafson, K. J. (2008). Fascicular perineurium thickness, size, and position affect model predictions of neural excitation. IEEE Trans Neural Syst Rehabil Eng, 16(6):572-81.

[Hadjinicolaou et al., 2015] Hadjinicolaou, A. E., Meffin, H., Maturana, M. I., Cloherty, S. L., and Ibbotson, M. R. (2015). Prosthetic vision: devices, patient outcomes and retinal research. Clin Exp Optom, 98(5):395-410.

[Hebert et al., 2014] Hebert, J. S., Olson, J. L., Morhart, M. J., Dawson, M. R., Marasco, P. D., Kuiken, T. A., and Chan, K. (2014). Novel targeted sensory reinnervation technique to restore functional hand sensation after transhumeral amputation. IEEE Trans Neural Syst Rehabil Eng, 22(4):765-73.

[Jackson et al., 2006a] Jackson, A., Mavoori, J., and Fetz, E. E. (2006a). Long-term motor cortex plasticity induced by an electronic neural implant. 444(7115):56-60.

[Jackson et al., 2006b] Jackson, A., Moritz, C., Mavoori, J., Lucas, T., and Fetz, E. (2006b). The neurochip BCI: towards a neural prosthesis for upper limb function. Ieee T Neur Sys Reh, 14(2):187-190.

[Johansson and Flanagan, 2009] Johansson, R. and Flanagan, J. (2009). Coding and use of tactile signals from the fingertips in object manipulation tasks. Nature Reviews Neuroscience.

[Johansson and Vallbo, 1979] Johansson, R. and Vallbo, A. (1979). Tactile sensibility in the human hand: relative and absolute densities of four types of mechanoreceptive units in glabrous skin. J Physiology, 286(1):283-300.

[Johansson and Westling, 1984] Johansson, R. and Westling, G. (1984). Roles of glabrous skin receptors and sensorimotor memory in automatic control of precision grip when lifting rougher or more slippery objects. Exp Brain Res, 56(3).

[Kejlaa, 1993] Kejlaa, G. (1993). Consumer concerns and the functional value of prostheses to upper limb amputees. Prosthetics and orthotics international. 
[Kiper et al., 2007] Kiper, D., Martin, K., and Scherberger, H. (2007). Cortical plasticity: A view from nonhuman primates. Gwumc Dept, 4(1):34-42.

[Koyama et al., 2010] Koyama, S., Chase, S., Whitford, A., Velliste, M., Schwartz, A., and Kass, R. (2010). Comparison of braincomputer interface decoding algorithms in open-loop and closed-loop control. J Comput Neurosci, 29(1-2):73-87.

[Kuiken et al., 1995] Kuiken, T., Childress, D., and Rymer, W. (1995). The hyperreinnervation of rat skeletal muscle. Brain Res, 676(1):113-123.

[Liu et al., 2014] Liu, J., Feng, F., and Nakamura, Y. (2014). A taxonomy of everyday grasps in action. (Humanoids).

[Logothetis et al., 2001] Logothetis, N., Pauls, J., Augath, M., and Trinath, T. (2001). Neurophysiological investigation of the basis of the fMRI signal. Nature.

[Marieb and Hoehn, 2007] Marieb, E. and Hoehn, K. (2007). Human anatomy \& physiology.

[Marshall, 2007] Marshall, G. (2007). Proceedings of the 2007 Animal-Borne Imaging Symposium, pages 183-192. National Geographic Society.

[Marshall et al., 2005] Marshall, G. J., Bakhtiari, M., and Rasch, D. J. (2005). Terrestrial crittercam system, US 2005/0162279 a1.

[Medina et al., 2012] Medina, L., Lebedev, M., Joseph, O., and Nicolelis, M. (2012). Stochastic facilitation of artificial tactile sensation in primates. J Neurosci, 32(41):14271-14275.

[Menz et al., 2015] Menz, V., Schaffelhofer, S., and Scherberger, H. (2015). Representation of continuous hand and arm movements in macaque areas m1, f5, and AIP: a comparative decoding study. J Neural Eng, 12(5):056016.

[Micera et al., 2010] Micera, S., Citi, L., Rigosa, J., Carpaneto, J., Raspopovic, S., Pino, G., Rossini, L., Yoshida, K., Denaro, L., Dario, P., and Rossini, P. (2010). Decoding information from neural signals recorded using intraneural electrodes: Toward the development of a neurocontrolled hand prosthesis. P Ieee, 98(3):407-417. 
[Micera et al., 2011] Micera, S., Rossini, P., Rigosa, J., Citi, L., Carpaneto, J., Raspopovic, S., Tombini, M., Cipriani, C., Assenza, G., Carrozza, M., Hoffmann, K., Yoshida, K., Navarro, X., and Dario, P. (2011). Decoding of grasping information from neural signals recorded using peripheral intrafascicular interfaces. J Neuroeng Rehabil, 8(1):1-10.

[Morel et al., 2015] Morel, P., Ferrea, E., Bahareh, T., Audí, J., Ruff, R., Hoffmann, K., Lewis, S., Russold, M., Dietl, H., Lait, A., Schroeder, D., Krautschneider, W., Meiners, T., and Gail, A. (2015). Long-term decoding of movement force and direction with a wireless myoelectric implant. J Neural Eng, 13(1):016002.

[Murata et al., 1997] Murata, A., Fadiga, L., and Fogassi, L. (1997). Object representation in the ventral premotor cortex (area f5) of the monkey. Journal of .

[Navarro et al., 2005] Navarro, X., Krueger, T., and Lago, N. (2005). A critical review of interfaces with the peripheral nervous system for the control of neuroprostheses and hybrid bionic systems. Journal of the .

[Oddo et al., 2016] Oddo, C., Raspopovic, S., Artoni, F., and Mazzoni, A. (2016). Intraneural stimulation elicits discrimination of textural features by artificial fingertip in intact and amputee humans. Elife.

[Paul and Kercel, 2003] Paul, B. and Kercel, S. W. (2003). Sensory substitution and the humanmachine interface. 7(12):541-546.

[Pruszynski and Johansson, 2014] Pruszynski, A. J. and Johansson, R. S. (2014). Edgeorientation processing in first-order tactile neurons. 17(10):1404-1409.

[Quiroga et al., 2006] Quiroga, R., Nadasdy, Z., and Y., B. (2006). Unsupervised spike detection and sorting with wavelets and superparamagnetic clustering. Neural Comput, 16(8):1661-1687.

[Raspopovic et al., 2012] Raspopovic, S., Capogrosso, M., Badia, J., Navarro, X., and Micera, S. (2012). Experimental validation of a hybrid computational model for selective stimulation using transverse intrafascicular multichannel electrodes. Ieee T Neur Sys Reh, 20(3):395-404. 
[Raspopovic et al., 2011] Raspopovic, S., Capogrosso, M., and Micera, S. (2011). A computational model for the stimulation of rat sciatic nerve using a transverse intrafascicular multichannel electrode. Ieee T Neur Sys Reh, 19(4):333-344.

[Raspopovic et al., 2014] Raspopovic, S., Capogrosso, M., Petrini, F., Bonizzato, M., Rigosa, J., Pino, G., Carpaneto, J., Controzzi, M., Boretius, T., Fernandez, E., Granata, G., Oddo, C., Citi, L., Ciancio, A., Cipriani, C., Carrozza, M., Jensen, W., Guglielmelli, E., Stieglitz, T., Rossini, P., and Micera, S. (2014). Restoring natural sensory feedback in Real-Time bidirectional hand prostheses. 6(222):222ra19-222ra19.

[Raspopovic et al., 2010] Raspopovic, S., Carpaneto, J., Udina, E., Navarro, X., and Micera, S. (2010). On the identification of sensory information from mixed nerves by using singlechannel cuff electrodes. J Neuroeng Rehabil, 7(1):1-15.

[Rice et al., 2015] Rice, K., Scott, A., and Guyot, A. (2015). Detection of Position-Related sciatic nerve dysfunction by somatosensory evoked potentials during spinal surgery. Neurodiagnostic $J, 55(2): 82-90$.

[Roche et al., 2014] Roche, A., Rehbaum, H., Farina, D., and Aszmann, O. (2014). Prosthetic myoelectric control strategies: A clinical perspective. Curr Surg Reports, 2(3).

[Romo et al., 1998] Romo, R., Hernández, A., Zainos, A., and Salinas, E. (1998). Somatosensory discrimination based on cortical microstimulation. Nature, 392(6674):387-390.

[Romo and Salinas, 2001] Romo, R. and Salinas, E. (2001). Touch and go: decision-making mechanisms in somatosensation. Annual review of neuroscience.

[Romo and Salinas, 2003] Romo, R. and Salinas, E. (2003). Flutter discrimination: neural codes, perception, memory and decision making. Nature Reviews Neuroscience.

[Rossini et al., 2010] Rossini, P., Micera, S., Benvenuto, A., Carpaneto, J., Cavallo, G., Citi, L., Cipriani, C., Denaro, L., Denaro, V., Pino, G., Ferreri, F., Guglielmelli, E., Hoffmann, K., Raspopovic, S., Rigosa, J., Rossini, L., Tombini, M., and Dario, P. (2010). Double nerve intraneural interface implant on a human amputee for robotic hand control. Clin Neurophysiol, 121(5):777-783. 
[Sahin and Durand, 1997] Sahin, M. and Durand, D. (1997). An interface for nerve recording and stimulation with cuff electrodes. in Medicine and Biology Society.

[Schaffelhofer et al., 2015] Schaffelhofer, S., Andres, A., and Scherberger, H. (2015). Decoding a wide range of hand configurations from macaque motor, premotor, and parietal cortices. $J$ Neurosci, 35(3):1068-1081.

[Schaffelhofer and Scherberger, 2012] Schaffelhofer, S. and Scherberger, H. (2012). A new method of accurate hand- and arm-tracking for small primates. J Neural Eng, 9(2):026025.

[Scherberger, 2009] Scherberger, H. (2009). Neural control of motor prostheses. Curr Opin Neurobiol, 19(6):629-633.

[Scherberger et al., 2003] Scherberger, H., Fineman, I., Musallam, S., Dubowitz, D., Bernheim, K., Pesaran, B., Corneil, B., Gilliken, B., and Andersen, R. (2003). Magnetic resonance image-guided implantation of chronic recording electrodes in the macaque intraparietal sulcus. J Neurosci Meth, 130(1):1-8.

[Spüler et al., 2014] Spüler, M., Walter, A., A, R., Naros, G., Birbaumer, N., Gharabaghi, A., Rosenstiel, W., and Bogdan, M. (2014). Decoding of motor intentions from epidural ECoG recordings in severely paralyzed chronic stroke patients. 11(6):066008.

[Stetson et al., 1992] Stetson, D., Albers, J., Silverstein, B., and Wolfe, R. (1992). Effects of age, sex, and anthropometric factors on nerve conduction measures. Muscle Nerve, 15(10):1095-1104.

[Sunderland, 1945] Sunderland, S. (1945). The innervation of the flexor digitorum profundus and lumbrical muscles. Anatomical Rec, 93(4):317-321.

[Sussillo et al., 2015] Sussillo, D., Churchland, M., Kaufman, M., and Shenoy, K. (2015). A neural network that finds a naturalistic solution for the production of muscle activity. Nat Neurosci, 18(7):1025-1033. 
[Tabot et al., 2013] Tabot, G., Dammann, J., Berg, J., Tenore, F., Boback, J., Vogelstein, R., and Bensmaia, S. (2013). Restoring the sense of touch with a prosthetic hand through a brain interface. Proc National Acad Sci, 110(45):18279-18284.

[Tabot et al., 2015] Tabot, G., Kim, S., Winberry, J., and Bensmaia, S. (2015). Restoring tactile and proprioceptive sensation through a brain interface. Neurobiol Dis, 83:191-198.

[Tan et al., 2015] Tan, D., Schiefer, M., Keith, M., Anderson, J., and Tyler, D. (2015). Stability and selectivity of a chronic, multi-contact cuff electrode for sensory stimulation in human amputees. J Neural Eng, 12(2):026002.

[Tan et al., 2014] Tan, D., Schiefer, M., Keith, M., Anderson, J., Tyler, J., and Tyler, D. (2014). A neural interface provides long-term stable natural touch perception. Sci Transl Med, 6(257):257ra138-257ra138.

[Townsend et al., 2008] Townsend, B., Subasi, E., and Scherberger, H. (2008). Real time decoding of hand grasping signals from macaque premotor and parietal cortex. Biomed Eng.

[Unknown, 2017] Unknown, A. (2017). Arduino low power - how to run ATmega328P for a year on coin cell battery.

[Welch and Bishop, 1995] Welch, G. and Bishop, G. (1995). An introduction to the kalman filter.

[Zuo and Olson, 2014] Zuo, K. and Olson, J. (2014). The evolution of functional hand replacement: From iron prostheses to hand transplantation. Plastic Surgery. 

Appendix C

Curriculum Vitae 


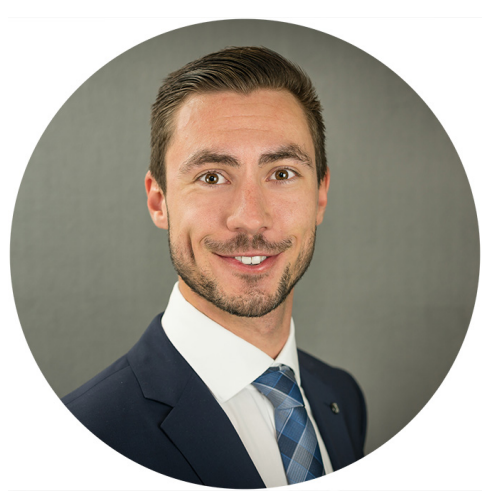

\section{Education}

\section{Address}

Barfüßerstraße 16

37073 Göttingen

Germany

\section{Nationality}

Dutch

Date of Birth: 28-12-1989

\section{Contact}

+4915773922965

jeroen.buil@gmail.com

\section{Web}

www.linkedin.com/in/jeroen-buil

\section{Personal Skills}

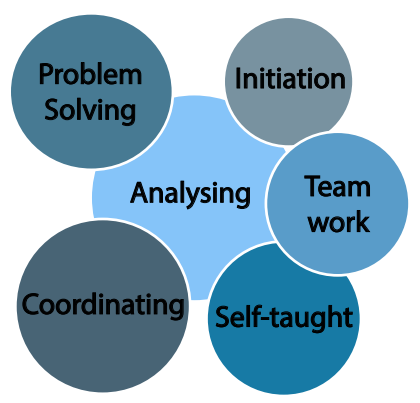

\section{Programming}

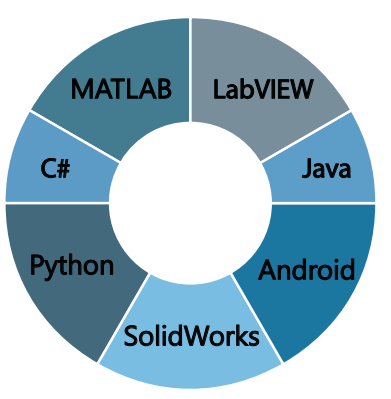

\section{Jeroen Buil}

(Neuro)Engineer

PhD student in Biomedical Engineering and Neuroscience, with experience in the design and development of (neuroprosthetic) prototypes. I characterise myself as analytical, versatile and creative.

Dec. 2013 - Present

PhD Systems Neuroscience Neurobiology Laboratory, German Primate Center (DPZ)

Thesis title: 'Peripheral nervous system control for neuroprostheses' Evaluated a new type of neural interface for the control of robotic hands and the ability to give sensory feedback to the missing limbs of amputee patients.

Sep. 2011 - Jul. 2013

MSc Biomedical Engineering - Cum Laude Department of Information Technology and Electrical Engineering

Specialisation: Bio-electronics

Thesis topic: hand motorics rehabilitation for stroke patients

Sep. 2008 - Jul. 201

BSc Biomedical Engineering - Cum Laude Department of Biomedical Engineering

Thesis topic: development of a vestibular prosthesis

Minor - Neuroscience

School of Biomedical Sciences

University of Nottingham, UK

Semester Abroad - Fall Semester 2009

\section{Awards and Honours}

Sep. 201

VSB (United Savings Banks) Fund Scholarship

This is amongst the most coveted scholarships available in The Netherlands, intended for Dutch citizens planning to study abroad.

Jul. 2011

Letter of Recommendation from the Rector Magnificus of Eindhoven University of Technology

Rewarded for successfully finishing the Bachelor with an Honours Degree.

Jul. $2011 \quad$ Honours Diploma

Rewarded for successfully finishing the Honours Programme. 


\section{Languages}

- Dutch: mother tongue

- English: fluent

- German: proficient

- French: basic

\section{Interests}

- Travelling

- Photo/videography

- Various sports:

- Swimming + Diving

- Wave/Wind/Kite-surfing

- Hiking

- Playing the piano

- Tinkering with electronics

\section{Additional Info}

Willing to relocate for work

\section{Experience \& Other Activities}

Jan. 2014 - Present

Jun. 2016 - Jun. 2017

May. 2015 - Jun. 2017

Sep. 2012 - Feb. 2013 Teaching assistant

Assisted in the courses 'Motor Systems', 'Application Oriented Programming Using MATLAB' and 'Introduction to Programming in Java'.

Feb. 2011 - Sep. 2011 Research assistant Instrument Development Engineering \& Evaluation (IDEE)

- Assisted in the vestibular implant research in cooperation with the Academic Hospital Maastricht.

- Responsible for the development of a system for stimulating of the vestibular nerve and analysis of the Vestibulo-Ocular-Reflex (VOR).

Sep. 2009 - Jul. 2011

\section{Honours Programme student}

Eindhoven University of Technology The Honours Programme is an additional program, for motivated and talented undergraduate students, characterised by intensive lectures and projects on an interdisciplinary subject matter given by top researchers.

Sep. 2009 - Jul. 2011

Volunteer promotional team

Eindhoven University of Technology Promotion work (organising workshop and open days) to raise enthusiasm among high school students to follow a career path in engineering.

Sep. 2009 - Jul. 2010

Board member - Travel commission

Honours Programme

Board member of the Honours Programme Travel Commission organising a nuclear physics focused study trip to France and Switzerland.

\section{Personal Skills and Competences}

\section{Analytical}

- With a great deal of experience in designing experimental setups and developing prototypes, I learned to pinpoint the strengths and potential weak points in strategies and designs.

\section{Versatile}

- My broad engineering and medical background, and my eagerness to learn new skills, allow me to tackle a wide range of challenges. Also, years of living and travelling abroad greatly improved my intercultural skills and my ability to adapt to different environments.

\section{Creative}

- I get a lot of enjoyment out of finding new solutions or ways to improving current ones. This is reflected in the professional projects I worked on, as well as my choice of hobbies.

\section{Computer skills}

- Programming: Highly competent user of programming languages, such as MATLAB and LabVIEW. Also competent in Python, Java, C\#, and Android development.

- Graphical Design: Proficient user of Solidworks for Computer Aided Design (CAD)-drawings. 
